\title{
ÜBER DEN IDEALEN RAND UND EINIGE SEINER ANWENDUNGEN BEI DER IKLASSIFIKATION DER RIEMANNSCHEN FLÄCHEN
}

\author{
CORNELIU CONSTANTINESCU UND AUREL CORNEA
}

\section{Einleitung}

Ein gewöhnliches Verfahren bei dem Studium der Riemannschen Flächen besteht darin, dass man die für die komplexe Ebene benutzten Methoden auf allgemeine Riemannsche Flächen überführt und die bekannten Sätze für schlichtartige Gebiete auf den Riemannschen Flächen verallgemeinert. Dises Verfahren schlägt zuweilen fehl, da manche Sätze, welche im Falle der schlichtartigen Gebiete richtig sind, im Falle beliebiger Riemannscher Flächen nicht mehr wahr bleiben. So war, zum Beispiel, die Gleichheit $O_{G}=O_{H B}$, im Falle der schlichtartigen Flächen richtig, wogegen sie aber für beliebige Riemannsche Flächen durch die Beziehung $O_{G} \subset O_{H B}$ ersetzt werden musste. Inwiefern man erwartet, einen, für die schlichtartigen Flächen bekannten Satz, auf den Riemannschen Flächen wiederzufinden, hat eine solche Lage einen gewissermassen paradoxen Charakter. Um die vollständige Klarheit wiederherzustellen, drängt sich die Notwendigkeit eines Studiums der Uhrsachen auf, die die Erscheinung des Paradoxons veranlasst haben.

Vorliegende Arbeit entspringt einer neuerschienenen Arbeit von Kuramochi [6], welcher einen solchen Paradoxon besonders herausgehoben hat. Und zwar hat Kuramochi gezeigt, dass wenn eine Riemannsche Fläche $R$ der Klasse $O_{H i}$ $-O_{G}$ (bzw. $O_{H D}-O_{G}$ ) angehört, so gehört $R-K \operatorname{der}$ Klasse $O_{A B B}\left(\right.$ bzw. $O_{A D}$ ) an, wo $K$ eine beliebige kompakte Menge auf $R$ ist, welche den Zusammenhang der Fläche $R$ nicht zerstört. Bei den schlichtartigen Riemannschen Flächen war die Zugehörigkeit zu der Klasse $O_{A B}\left(\mathrm{bzw}, O_{A 7}\right.$ ) eine Folge der "schwäche" des Randes. Als Sario [19] bei dem 11 Skandinavischen Kongress der Mathematiker ein allgemeines Prinzip für die Klassifikation der Riemannschen Flächen eingeführt hat, wurde er von der Idee geleitet, die Riemanschen Flächen, in

Received September 6, 1957. 
Bezug auf eine bestimmte Klasse von Funktionen, in Flächen mit starkem bzw. schwachem Rande zu teilen: "Comme critère naturel pour la classification des surfaces de Riemann il s'offre ici la "force" de la frontière d'une surface ouverte. On divise l'ensemble des surfaces de manières diverses, en surfaces avec une frontière "plus faible" et "plus forte." " Das Beispiel von Kuramochi hat eine umfangreiche Klasse von Riemannschen Flächen aus der Klasse $O_{A B}$ (bzw. $O_{A D}$ ) gezeigt, welche einen starken Rand besitzen. Solch ein Beispiel ist shon früher von Myrberg P. J. [14] gegeben worden, jedoch sind die Gründe aus welchen eine Riemannsche Fläche mit starkem Rande der Klasse $O_{A B}$ (bzw. $O_{A D}$ ) angehören, bei Kuramochi und Myrberg verschieden.

In der vorliegenden Arbeit beabsichtigen wir, die Gründe des von Kuramochi herausgehobenen Paradoxons zu untersuchen. Dazu werden wir den Begriff eines Punktes des idealen Randes einer Riemannschen Fläche einführen. Nicht die Punkte, sondern die Mengen von Punkten des idealen Randes werden für uns wichtig sein. Alle Betrachtungen werden ständig mit der Vernachlässigung der Mengen vom harmonischen Masse Null gemacht, denn das erleichtert die Beweise, und die Ergebnisse, die wir im Sinne haben, werden dadurch nicht entstellt. Als Grund des obenerwähnten Paradoxons wird sich eine bestimmte Eigenschaft der Unteilbarkeit einiger Mengen von Punkten des idealen Randes zeigen. Es gibt Mengen von positivem Masse im Rande einiger Riemannschen Flächen, welche keinerlei Zerlegung in zwei messbare punktfremde Mengen mit positivem Masse erlauben. Die Anwesenheit einer sochen Menge im Rande einer Riemannschen Fläche $R$ hat als Folge, dass $R$ der Klasse $O_{A B}$ angehört. Etwas ähnliches wird auch für die Klasse $O_{A n}$ durchgeführt. Diese "unteilbaren" Mengen werden in eine enge Beziehung $\mathrm{zu}$ den minimalen Funktionen einer Klasse von positiven harmonischen Funktionen gebracht.

Im ersten Abschnitt führen wir die Punkte des idealen Randes einer Riemannschen Fläche $R$ ein; sie werden ungefähr die Äquivalenzklassen der Punkte $t,|t|=1$, in Bezug auf die Deckbewegungsgruppe des Kreises $|t|<1$, welcher als universelle Überlagerungsfläche von $R$ betrachtet wird, sein. Im zweiten Abschnitt wird der Begriff von unteilbaren Mengen eingeführt, und die Klasse der Riemannschen Fläche, welche eine solche Menge auf ihrem idealen Rande enthalten, untersucht. Damit wir die tieferen Eigenschaften dieser Klasse erhalten können, welche im Abschnitt $V$ untersucht werden, bringen wir ịm 
dritten Abschnitt die von Kuramochi eingeführten Extremisierungs und Inextremisierungs-Operationen [5], und zeigen ihre wichtigsten Eigenschaften. Von grosser Nützlichkeit hat sich der Übergang auf die universelle Überlagerungsfläche $|t|<1$ und die Darstellung dieser Operationen durch das PoissonLebesguesche Integral erwiesen. Im vierten Abschnitt wird der Begriff der $H D$-Unteilbarkeit eingeführt, damit die Ergebnisse, die für die beschränkten Funktionen gefunden wurden, auch über die Klasse der Funktionen mit beschränktem Dirichlet Integral erweitert werden können. Im fünften Abschnitt werden wir die Eigenschaften der Riemannschen Flächen, welche im Rande unteilbare (bzw. $H D$-unteilbare) Mengen enthalten, gründlicher untersuchen und einige Sätze von Kuramochi [6] und Kuroda [8] verallgemeinern.

\section{Der ideale Rand einer Riemannschen Fläche}

1. Es sei $R$ eine Riemannsche Fläche und $z=\varphi(t)$ die eineindeutige und konforme Abbildung des Kreises $|t|<1$ auf die universelle Überlagerungsfläche von $R$. $\quad\left\{t_{\text {, }}\right\}$ seien die Punkte, die in $z_{0} \in R$ abgebildet werden. Falls $R$ nicht nullberandet ist, was wir in Folgendem immer annehmen werden, so ist die Summe

$$
\sum_{i=1}^{\infty}(1-\mid t, i)
$$

konvergent und für die Greensche Funktion $g\left(z, z_{0}\right)$ der Fläche $R$ besteht die Gleichheit

$$
g\left(\varphi(t), z_{0}\right)=\sum_{\nu=1}^{\infty} \log \frac{1-t \bar{t}_{0}}{t-t_{\nu}}
$$

([16], Seite 336-338). Wegen der Konvergenz der obigen Summe ist das BlaschkeProdukt

$$
\pi(t)=\prod_{\nu=1}^{\infty} \frac{t-t_{\nu}}{t \bar{t}_{\nu}-1} \bar{t}_{t_{\nu}}
$$

konvergent und es gilt

$$
g\left(\varphi(t), z_{0}\right)=\log \frac{1}{|\pi(t)|}
$$

Wir bezeichnen mit $\tilde{f}$ die Menge der Punkte $e^{i \theta}$, für welche $g\left(c(t), z_{0}\right)$ den Winkelgrenzwert Null hat. Aus einer uns bekannten Eigenschaft des BlaschkeProduktes folgt, dass $\widetilde{f}$ das Mass $2 \pi$ hat ([15], Seite 207). 
Von einer Kurve $\lambda: t=t(\tau)(0 \leqq \tau<1 ;|t(\tau)|<1)$ sagen wir, dass sie in $e^{i \theta}$ einen Winkeleingang hat, wenn

$$
\lim _{\tau \rightarrow 1} t(\tau)=e^{i 0}
$$

ist und wenn man ein $\varepsilon>0$ so finden kann, dass

$$
\arg \frac{e^{i \theta}-t(\tau)}{e^{i \theta}}<\frac{\pi}{2}-\varepsilon
$$

ist. Wenn $e^{i \theta}$ der Menge $\tilde{j}$ angehört, und $A: z=\varphi(t(\tau))$ das Bild von $\lambda$ auf $R$ ist, so muss 1 gegen den idealen Rand der Fläche $R$ streben; d.h., dass man für jede kompakte Menge $K$ ein $\tau_{K}<1$ finden kann, so dass für $\tau>\tau_{K}$, $\varphi(t(\tau)) \notin K$. Das folgt aus der Tatsache, dass auf $A$ die Greensche Funktion gegen Null strebt:

$$
\lim _{\tau \rightarrow 1} g\left(\varphi(t(\tau)), z_{0}\right)=0
$$

Es seien $z_{0}$ und $z_{0}^{\prime}$ zwei beliebige Punkte auf $R$ und $G$ ein Jordansches Gebiet, welches $z_{0}$ und $z_{0}^{\prime}$ enthält. Hier und weiter werden wir die Bezeichnung j $G$ für den Rand von $G$ benutzen. Wenn

$$
\begin{aligned}
& m=\inf _{z \in \partial(i} g\left(z, z_{0}\right)>0 ; M=\sup _{z \in \partial G} g\left(z, z_{0}\right)<\infty, \\
& m^{\prime}=\inf _{z \in \partial F_{i}} g\left(z, z_{0}^{\prime}\right)>0 ; M^{\prime}=\sup _{z \in \partial G i} g\left(z, z_{0}^{\prime}\right)<\infty
\end{aligned}
$$

ist, so folgt aus dem Maximumprinzip, dass

$$
\begin{gathered}
\frac{g\left(z, z_{0}\right)}{M} \leqq \frac{g\left(z, z_{0}^{\prime}\right)}{m^{\prime}}, \\
g\left(z, z_{0}^{\prime}\right) \leqq \frac{g\left(z, z_{0}\right)}{M^{\prime}} \leqq \frac{m}{m}
\end{gathered}
$$

für $z \in R-\bar{G}$ ist. Daraus ist $z$ ersehen, dass $g\left(z, z_{0}\right)$ und $g\left(z, z_{0}^{\prime}\right)$ gleichzeitig auf 1 gegen Null streben. Die Menge $\mathfrak{f}$ hängt somit vom Punkte $z_{0}$ nicht ab.

Es sei $(T)$ die Deckbewegungsgruppe der Überlagerung $z=\varphi(t) . \quad T \in(T)$ ist eine eineindeutige Abbildung des Kreises $|t|<1$, für welche $\varphi(T(t)) \equiv \varphi(t)$ ist. Die Menge $\tilde{y}$ ist offenbar automorph in Bezug auf $(T)$, d.h. es ist $\hat{\jmath}=T(\hat{j})$ für jedes $T \in(T)$, weil $\pi(t)$; automorph in Bezug auf $(T)$ ist. Die Deckbewegungsgruppe teilt die Menge $\tilde{j}$ in Äquivalenzklassen: zwei Punkte $t_{1}, t_{2}$ der Menge $\tilde{j}$ heissen äquivalent in Bezug auf $(T)$, wenn man ein $T \in(T)$ finden kann, für welches $t_{2}=T\left(t_{1}\right)$ ist. Eine Äquivalenzklasse $p$ der Menge $\widetilde{v}$ wird 
ein Punkt des idealen Randes der Riemannschen Fläche $R$ genannt und die Menge $p$. der Punkte, welche der Äquivalenzklasse angehören, heisst das Bild von $p$. Eine Menge $M$ von Punkten des idealen Randes ist eine Menge von Äquivalenzklassen und die Menge $\mathfrak{M}$ der Punkte aus $\mathfrak{F}$, welche diesen Äquivalenzklassen angehören, heisst das Bild von $M$. Die Menge aller Punkte des idealen Randes werden wir mit $F$ bezeichnen; is ist offenbar das Bild von $F$.

2. Wir führen wie gewöhnlich ([22], Seite 85-87) die idealen Randkomponenten ein. Es sei auf $R$ eine Folge von Gebieten $\left\{G_{n}\right\}$ gegeben, welche folgende Bedingungen erfillen:

1. Der Rand von $G_{n}$ besteht aus einer einzigen Jordankurve

2. Für jedes $n$ ist $\bar{G}_{n+1} \subset G_{n}$.

3. Es ist

$$
\bigcap_{n=1}^{\infty} G_{n}=\phi
$$

Zwei solcher Folgen $\left\{G_{n}\right\},\left\{G_{n}^{\prime}\right\}$ werden äquivalent genannt, wenn man für jedes $n$ ein $N_{n}$ finden kann, so dass $G_{m} \subseteq G_{n}^{\prime}, G_{m}^{\prime} \cong G_{n}$ für $m \geqq N_{n}$ ist. Die Menge der Folgen $\left\{G_{n}\right\}$, welche die Bedingungen 1-3 erfüllen, zerfällt in Äquivalenzklassen. Eine Äquivalenzklasse $a$ wird eine ideale Randkomponente genannt (élément-frontière) und jede Foíge $\left\{C_{i}\right\}$ aus der Äquivalenzklasse $a$ wird determinante Folge von $a$ genannt.

Es sei $a$ eine ideale Randkomponente und $\left\{G_{n}\right\}$ eine determinante Folge von $a$. Man sagt, dass die Punkte $s, \in R(\nu=1,2, \ldots)$ gegen $a$ konvergieren, wenn man für jedes $n$ ein $\nu(n)$ finden kann, so dass $z_{\text {, }} \in G_{n}$ für $\nu \geqslant \nu(n)$. Ähnlicherweise sagt man, dass eine Kurve $1: z=z(\tau) \quad(0 \leqq \tau<1)$ gegen $a$ strebt, wenn man für jedes $n$ ein $\tau(n)<1$ finden kann, so dass $z(\tau) \in G_{n}$ für $\tau \gtrsim \tau(n)$. Beide Definitionen sind von der Auswahl der determinanten Folge $\left\{G_{n}\right\}$ von $a$ unabhängig. Es ist leicht $z u$ ersehen, dass man aus jeder Folge $\left\{z_{i}\right\}, z_{3} \in R$, eine Teilfolge wählen kann, die entweder gegen einen Punkt $z_{0} \in R$ oder gegen eine ideale Randkomponente konvergiert. Jede Kurve $A$, die gegen den idealen Rand der Fläche $R$ strebt, strebt gegen eine bestimmte ideale Randkomponente.

Jedem Punkte $p$ des idealen Randes wird eine ideale Randkomponente $a$ zugeordnet. Das geschiet so: man nimmt einen beliebigen Punkt $e^{i \theta}$, welcher der Äquivalenzklasse $p$ angehört, $e^{\prime \theta} \in b$, und eine beliebige Kurve $\lambda$ im Kreise 
$|t|<1$, die in $e^{i 0}$ einen Winkeleingang hat. Es hat sich gezeigt, dass das Bild $A$ von $\lambda$ auf $R$ gegen eine bestimmte ideale Randkomponente $a$ strebt. Man sagt, dass $p$ der ideale Randkomponente $a$ angehört, oder, dass der Punkt $p$ auf $a$ liegt. Es ist zu zeigen, dass $a$ eindeutig durch $p$ bestimmt ist, d.h. nicht von $\operatorname{der}$ Auswahl von $e^{i 0}$ und $\lambda$ abhängt. Es sei $e^{i 0^{\prime}}$ ein beliebiger Punkt aus $\mathfrak{p}$ und $\lambda^{\prime}$ eine beliebige Kurve im Kreise $|t|<1$, die in $e^{i 0^{\prime}}$ einen Winkeleingang hat. Wir werden zeigen, dass das Bild $A^{\prime}$ von $\lambda^{\prime}$ auf $R$ gegen dieselbe ideale Randkomponente $a$ strebt. Da $e^{i \theta}$ und $e^{i 0^{\prime}}$ äquivalent in Bezug auf $(T)$ sind, so kann man eine Deckbewegung $T \in(T)$ finden, so dass

$$
e^{i \theta}=T\left(e^{i \theta^{\prime}}\right)
$$

ist. Mittels $T$ geht die Kurve $\lambda^{\prime}$ in eine neue Kurve $\lambda^{\prime \prime}=T\left(\lambda^{\prime}\right)$ über, welche in $e^{i \theta}$ einen Winkeleingang hat. $\lambda^{\prime}$ und $\lambda^{\prime \prime}$ haben dasselbe Bild $\Lambda^{\prime}$ auf $R$. Deformiert man stetig $\lambda^{\prime \prime}$ in $\lambda$, so dass während der Deformation $\lambda^{\prime \prime}$ fortwährend einen Winkeleingang in $e^{i 0}$ hat, so strebt das Bild von $\lambda^{\prime \prime}$ auf $R$ während der Deformation ständig gegen den idealen Rand der Fläche $R$ und deshalb gegen ein und dieselbe ideale Randkomponente. Daraus ist $\mathrm{zu}$ ersehen, dass $A^{\prime}$ gegen $a$ strebt.

Jeder Punkt des idealen Randes gehört somit einer ideale Randkomponente an. Aber nicht jede ideale Randkomponente besitzt einen Punkt. Diese Tatsache wird aber im Folgenden keine Störungen mit sich bringen, denn wie soeben betont wurde, sind für uns nicht die Punkte, sondern die Mengen von Punkten des idealen Randes wichtig, und zwar nur solche, die positives harmonisches Mass haben.

3. Es sei $\Gamma$ eine geschlossene Jordankurve auf $R$, welche die Fläche in zwei Teile $R^{\prime}$ und $R^{\prime \prime}$ teilt. Weiter sei $a$ eine ideale Randkomponente und $\left\{G_{n}\right\}$ eine determinante Folge von a. Da $\Gamma$ eine kompakte Menge ist, so kann man ein $n(\Gamma)$ finden, so dass für $n>n(\Gamma) G_{n} \cap \Gamma=\phi$ ist. Daraus folgt, dass für $n>n(\Gamma), G_{n}$ entweder in $R^{\prime}$ oder in $R^{\prime \prime}$ enthalten ist. Falls $G_{n} \subset R^{\prime}$ ist und $\left\{G_{n}^{\prime}\right\}$ eine andere determinante Folge von $a$ ist, so ist $G_{n}^{\prime} \subset R^{\prime}$ für $n>n^{\prime}(\Gamma)$. Die Menge aller idealen Randkomponenten zerfällt somit in zwei Teilmengen, die wir mit $\beta^{\prime}$ und $\beta^{\prime \prime}$ bezeichnen, je nachdem $G_{n} \subset R^{\prime}$ oder $G_{n} \subset R^{\prime \prime}$ für genügend grosse $n$ ist. Wir werden die Mengen $\beta^{\prime}$ und $\beta^{\prime \prime}$ Portionen des idealen Randes nennen und sagen, dass $\beta^{\prime}$ (bzw. $\beta^{\prime \prime}$ ) die von dem Gebiete $R^{\prime}$ (bzw. $R^{\prime \prime}$ ) 
bestimmte Portion ist.

Es sei $\left\{R_{n}\right\}$ eine Ausschöpfung der Fläche $R$, so dass der Rand $\gamma_{n}$ von $R_{n}$ aus endlich vielen analytischen Jordankurven besteht und $\Gamma \subset R_{1}$ ist. Wir bezeichnen

$$
r_{n}^{\prime}=r_{n} \cap R^{\prime} ; r_{n}^{\prime \prime}=\gamma_{n} \cap R^{\prime \prime}
$$

$\omega_{n}(z)=\omega\left(z, \gamma_{n}^{\prime}, R_{n}\right)$ sei die in $\bar{R}_{n}$ harmonische Funktion, welche auf $\gamma_{n}^{\prime}$ den Wert 1 und auf $r_{n}^{\prime \prime}$ den Wert 0 annimmt. Wenn $g_{n}\left(z, z_{0}\right)$ die Greensche Funktion von $R_{n}$ ist, so ist

$$
\omega_{n}(z)=\frac{1}{2 \pi} \int_{i_{n}^{\prime}{ }^{\prime}} \frac{\partial g_{n}(\zeta, z)}{\partial n} \cdot d \zeta \mid
$$

Für $m<n$ ist $\gamma^{\prime} m$ homolog auf $R_{n}$ zu $\gamma_{n}^{\prime}$ und deshalb ist

$$
\omega_{n}(z)=\frac{1}{2 \pi} \int_{i_{m^{\prime}}} \frac{\partial g_{n}(\zeta, z)}{\partial n}|d \zeta|
$$

wenn $z \in R_{m}$. Für $n \rightarrow \infty$ folgt

$$
\begin{aligned}
\omega(z) & =\lim _{n \rightarrow \infty} \omega_{n}(z)=\lim _{n \rightarrow \infty} \frac{1}{2 \pi} \int_{\gamma_{m^{\prime}}} \frac{\partial g_{n}(\zeta, z)}{\partial n}|d \zeta|=\frac{1}{2 \pi} \int_{\tau^{\prime} m^{\prime}} \frac{\partial g(\zeta, z)}{\partial n}|d \zeta| \\
& =\lim _{m \rightarrow \infty} \frac{1}{2 \pi} \int_{r^{\prime} m^{\prime}} \frac{\partial g(\zeta, z)}{\partial n}\left|d \zeta=\frac{1}{2 \pi} \int_{\beta^{\prime}} \frac{\partial g(\zeta, z)}{\partial n}\right| d \zeta \mid
\end{aligned}
$$

für $z \in R$. Es sei $B^{\prime}$ (bzw. $B^{\prime \prime}$ ) die Menge der Punkte des idealen Randes, die auf idealen Randkomponenten aus der Portion $\beta^{\prime}$ (bzw. $\beta^{\prime \prime}$ ) liegen. Wir werden die harmonische Funktion $\omega\left(z, B^{\prime}, R\right)=\omega(z)$ das harmonische Mass der Menge $B^{\prime}$ oder das harmonische Mass der Portion $\hat{\beta}^{\prime}$ benennen.

Wir bezeichnen

$$
m=\inf _{z \in \Gamma} g\left(z, z_{0}\right)>0
$$

In $R_{n} \cap R^{\prime}$ (bzw. $\left.R_{n} \cap R^{\prime \prime}\right)$ ist $m\left(1-\omega_{n}(z)\right) \leqq g\left(z, z_{0}\right) \quad$ (bzw. $m \omega_{n}(z) \leqq g\left(z, z_{0}\right)$ ), da diese Ungleichung offenbar auf dem Rande erfüllt ist. Für $n \rightarrow \infty$ geht sie in

$$
m(1-\omega(z)) \leqq g\left(z, z_{0}\right) \quad\left(\text { bzw. } m \omega(z) \leqq g\left(z, z_{0}\right)\right)
$$

für $z \in R^{\prime}$ (bzw. $z \in R^{\prime \prime}$ ) über. Es sei jetzt $e^{i 0}$ ein Punkt der Menge $\mathbb{B}^{\prime}$ (bzw. $\left.\mathfrak{B}^{\prime \prime}\right)$, wo wir wie gewöhnlich mit $\mathfrak{B}^{\prime}$ das Bild der Menge $B^{\prime}$ bezeichnet haben, und $\lambda$ eine in $t<1$ gelegene Kurve, die in $e^{t 0}$ einen Winkeleingang hat. Auf $\lambda$ strebt $g\left(\varphi(t), z_{0}\right)$ gegen 0 , und aus der obigen Ungleichung sieht man, dass $\omega(c(t))$ gegen 1 (bzw. 0) strebt. Daraus folgt, dass die Funktion $\omega(\varphi(t))$ überall 
auf $\mathfrak{B}^{\prime}$ den Winkelgrenzwert 1 und überall auf $\mathfrak{B}^{\prime \prime}$ den Winkelgrenzwert 0 hat. Ähnlicherweise kann man leicht beweisen, dass falls $\omega(z)$ auf $A$ gegen 0 oder 1 strebt, so strebt $g\left(z, z_{0}\right)$ gegen 0 . Daraus folgt, dass $\mathfrak{B}^{\prime}$ (bzw. $\left.\mathfrak{B}^{\prime \prime}\right)$ mit der Menge von Punkten, wo $\omega(\varphi(t))$ den Winkelgrenzwert 1 (bzw. 0) hat, zusammenfällt. Die Mengen $\mathfrak{B}^{\prime}, \mathfrak{B}^{\prime \prime}$ sind somit im Lebesgueschen Sinne messbar und $\omega(\varphi(t))$ kann durch das Poisson-Lebesguesche Integral dargestellt werden

$$
\omega(\varphi(t))=\int_{\mathfrak{B}^{\prime}} P(\theta, t) d \theta
$$

wo

$$
P(\theta, t)=\frac{1}{2 \pi} \operatorname{Re} \frac{e^{i \theta}+t}{e^{i \theta}-t}
$$

ist.

Es sei $M$ eine Menge von Punkten des idealen Randes und $\mathfrak{M}$ ihr Bild auf $|t|=1$. Wir sagen, dass $M$ eine messbare Menge ist, wenn $\mathfrak{D}$ im Lebesgueschen Sinne messbar ist. Weiter sagen wir, dass $M$ nulles oder positives Mass hat, je rachdem das Lebesguesche Mass von $\mathfrak{M}$ null oder positiv ist. Die Menge der Punkte des idealen Randes, die auf den idealen Randkomponenten einer Portion liegen, ist somit messbar.

Es sei jetzt $a$ eine ideale Randkomponente, $A$ die Menge der Punkte des idealen Randes, die auf $a$ liegen, $\left\{G_{n}\right\}$ eine determinante Folge von $a, \beta_{n}$ die Portion des idealen Randes, die von dem Gebiete $G_{n}$ bestimmt ist und $B_{n}$ die Menge der Punkte des idealen Randes, welche auf idealen Randkomponenten aus $\beta_{n}$ liegen. Es ist offenbar

$$
A=\bigcap_{n=1}^{\infty} B_{n}, \quad \mathfrak{H}=\bigcap_{n=1}^{\infty} \mathfrak{B}_{n}
$$

wo $\mathfrak{A}$ bzw. $\mathfrak{B}_{n}$ das Bild der Menge $A$ bzw. $B_{n}$ ist. Da $\mathfrak{B}_{n}$ messbar ist, so ist auch $\mathfrak{A}$ messbar. Die Menge der Punkte des idealen Randes, welche auf einer idealen Randkomponente liegen, ist also messbar. Es ist $\omega\left(z, B_{n}, R\right)$ $\geq \omega\left(z, B_{n+1}, R\right)$. Wir werden die harmonische Funktion

$$
\omega(z, A, R)=\lim _{n \rightarrow \infty} \omega\left(z, B_{n}, R\right)
$$

das harmonische Mass der Menge $A$ oder das harmonische Mass der idealen Randkomponente $a$ benennen. Es ist

$$
\omega(\varphi(t), A, R)=\lim _{n \rightarrow \infty} \omega\left(\varphi(t), B_{n}, R\right)=\lim _{n \rightarrow \infty} \int_{\mathfrak{B}_{n}} P(\theta, t) d \theta=\int_{\mathfrak{M}} P(\theta, t) d \theta .
$$


Es sei $u^{*}\left(e^{i 0}\right)$ eine reelle summierbare Funktion

$$
\int_{0}^{2 \pi}\left|u^{*}\left(e^{i 0}\right)\right| d \theta<\infty
$$

welche automorph in Bezug auf $(T)$ ist: $u^{*}\left(T\left(e^{i 0}\right)\right)=u^{*}\left(e^{i \theta}\right)$ für jedes $T \in(T)$. Mittels des Poisson-Lebesgueschen Integrals bilden wir die harmonische Funktion

$$
u^{*}(t)=\int_{0}^{2 \pi} u^{*}\left(e^{i 0}\right) P(\theta, t) d \theta
$$

welche auch automorph in Bezug auf $(T)$ ist, denn $u^{*}(T(t))$ ist auch durch das Poisson-Lebesguesche Integral darstellbar und hat fast überall auf $|t|=1$. dieselben Winkelgrenzwerte wie $u(t)$. Daraus folgt, dass $u(z)=u^{*}\left(\varphi^{-1}(z)\right)$ eine eindeutige harmonische Funktion auf $R$ ist.

Für eine beliebige messbare Menge $M$ von Punkten des idealen Randes,

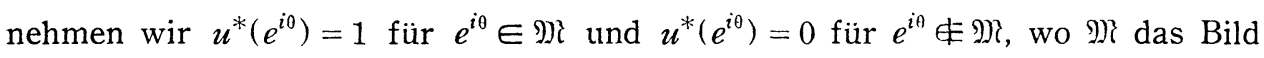
der Menge $M$ ist. Es ist dann

$$
u^{*}(t)=\int_{\mathfrak{M M}} P(\theta, t) d \theta
$$

Wir nennen die auf $R$ eindeutige und harmonische Funktion

$$
\omega(z, M, R)=u^{*}\left(\varphi^{-1}(z)\right)
$$

das harmonische Mass der Menge $M$. Es ist

$$
\omega(\varphi(t), M, R)=\int_{M_{i}} P(\theta, t) d \theta
$$

Fast überall auf $\mathfrak{M}$ hat $\omega(\varphi(t), M, R)$ den Winkelgrezwert 1 , und fast überall auf dem Komplement $C \mathfrak{M}$ von $\mathfrak{M}$ den Winkelgrezwert 0. Das harmonische Mass der Menge $M$ ist dann und nur dann null, wenn ihr Bild $\mathfrak{M}$ das Lebesguesche Mass Null hat.

4. SATz 1. Es sei $R$ eine nicht nullberandete Riemannsche Fläche, a eine ideale Randkomponente (bzw. $\beta$ eine Portion des idealen Randes) mit positivem harmonischem Masse und $w(z)$ eine auf $R$ meromorphe Funktion. Wenn für jede Kurve $.1: z=z(\tau) \quad(0 \leqq \tau<1)$, die gegen a strebt (bzw. die gegen eine ideale Randkomponente der Portion $\beta$ strebt) und auf welcher die Greensche Funktion gegen Null strebt 


$$
\lim _{\tau \rightarrow 1} w(z(\tau))=c
$$

ist, so ist $w(z) \equiv c$.

Es sei $A$ (bzw. $B$ ) die Menge der Punkte des idealen Randes, welche auf $a$ (bzw. auf idealen Randkomponenten der Portion $\beta$ ) liegen, und $\mathfrak{A}$ (bzw. $\mathfrak{B}$ ) ihr Bild. Die Menge $\mathfrak{N}$ (bzw. $\mathfrak{B}$ ) ist messbar und hat positives Lebesguesches Mass. Wir werden mit $\mathfrak{P}^{*}$ die Menge jener Punkte $e^{i 0}$ von $\mathfrak{A}$ bezeichnen, für die

$$
\lim _{i \rightarrow \Varangle e^{i \theta}} \omega(\varphi(t), A, R)=1
$$

ist; $\mathfrak{A}^{*}$ hat positives Mass. Es sei $e^{i \theta}$ ein Punkt der Menge $\mathfrak{I}^{*}$ (bzw. $\mathfrak{B}^{3}$ ) und $\lambda$ eine Kurve im Kreise $|t|<1$, die in $e^{i \theta}$ einen Winkeleingang hat. Ihr Bild $A$ durch $z=\varphi(t)$ auf $R$ strebt gegen $a$ (bzw. gegen eine ideale Randkomponente aus der Portion $\beta$ ), und die Greensche Funktion strebt auf $A$ gegen Null. Aus der Voraussetzung des Satzes folgt, dass $w(\varphi(t))$ auf $\lambda$ gegen $c$ strebt, und, da $\lambda$ beliebig gewählt wurde, dass $w(\varphi(t))$ in jedem Punkte der Menge $\mathfrak{I}^{*}$ (bzw. $\mathfrak{B}$ ) den Winkelgrenzwert $c$ hat. Aus dem Eindeutigkeitssatz von Priwaloff ([9], Seite 164) folgt dann $w(\varphi(t)) \equiv c$, was zu beweisen war.

\section{Unteilbare Mengen}

5. Es sei $R$ eine Riemannsche Fläche aus der Klasse $O_{H B}-O_{G}$ und $M_{1}, M_{2}$ zwei messbare Mengen des idealen Randes, für die $F=M_{1} \cup M_{2}, M_{1} \cap M_{2}=\phi$ ist. Dann muss wenigstens eine vom Masse Null sein, denn falls $\omega\left(z, M_{\nu}, R\right)>0$ $(\nu=1,2)$ ist, so ist $\sup _{z \in R} \omega\left(z, M_{\nu}, R\right)=1$ und da $R \in O_{H b}$, so folgt $\omega\left(z, M_{\nu}, R\right) \equiv 1$ Beide Funktionen können aber nicht gleich 1 sein, denn

$$
\omega\left(z, M_{1}, R\right)+\omega\left(z, M_{2}, R\right)=1
$$

Der ideale Rand einer Riemannschen Fläche $R \in O_{H B}$ ist also unteilbar: er kann nicht in zwei Mengen geteilt werden, so dass jede messbar und von positivem Masse sein soll. Diese Eigenschaft ist der wahre Grund der verschiedenen paradoxen Eigentümlichkeiten der Riemannschen Flächen der Klasse $O_{H B}-O_{G}$ ([16], Seite 322-324) [6]. Um grössere Allgemeinheit zu erlangen, führen wir folgende Definition ein.

Definition 1. Eine Menge $M$ von Punkten des idealen Randes heisst unteilbar, wenn sie messbar ist, positives harmonisches Mass hat und nicht in zwei messbare Mengen $M_{1}, M_{2}$ teilbar ist, so dass beide positives harmonisches. 
Mass haben. Anders ausgedrückt: $M$ heist unteilbar, wenn ihr Bild $\mathfrak{M}$ messbar und von positivem Masse ist, und wenn man nicht zwei automorphe messbare Mengen $\mathfrak{M}_{1}, \mathfrak{M}_{2}$ finden kann, so dass

$$
\mathfrak{M}=\mathfrak{M}_{1} \cup \mathfrak{M}_{2} ; \mathfrak{M}_{1} \cap \mathfrak{J}_{2}=\phi
$$

und beide positives Lebesguesches Mass haben.

Der ideale Rand einer Riemannschen Fläche enthält höchstens eine abzählbare Menge von unteilbaren Mengen.

Es sei $\left\{R_{n}\right\}$ eine Ausschöpfung der Fläche $R$, so dass der Rand $r_{n}$ von $R_{n}$ aus endlich vielen Jordankurven besteht

$$
r_{n}=\bigcup_{i=1}^{i n} r_{n i}
$$

Wir nehmen die Ausschöpfung so, dass jede Jordankurve $\gamma_{n i}$ die Fläche $R$ in $z$ wei Teile teilt und es sei $R_{n i}$ jener Teil, welcher $R_{n}$ nicht enthält. Mit $\beta_{n i}$ bezeichnen wir die von dem Gebiete $R_{n i}$ bestimmte Portion und mit $B_{n i}$ die Menge der Punkte des idealen Randes, welche auf idealen Randkomponenten der Portion $\beta_{n i}$ liegen; die Mengen $B_{n i}\left(i=1,2, \ldots, N_{n}\right)$ sind offenbar paarweise punktfremd. Es ist

$$
\mathfrak{R}=\bigcup_{i=1}^{N_{n}}\left(\mathfrak{M} \cap \mathfrak{B}_{n i}\right)
$$

wo $\mathfrak{B}_{n i}$ das Bild der Menge $B_{n i}$ ist. Ist $M$ unteilbar, so existiert ein $i_{n}$, so dass

$$
m(\mathfrak{N})=m\left(\mathfrak{M} \cap \mathfrak{B}_{n i_{n}}\right)
$$

ist, wo $m(\mathfrak{M})$ das Lebesguesche Mass der Menge $\mathfrak{M}$ ist, denn $\mathfrak{M} \cap \mathfrak{B}_{n i}$ sind automorphe Mengen lund nur eine von ihnen kann positives Mass haben. Es ist offenbar

$$
\beta_{n i_{n}} \supseteqq \beta_{n+1, i_{n+1}}
$$

Wir bezeichnen mit $a$ die ideale Randkomponente, die von der Folge $\left\{R_{n i_{n}}\right\}$ bestimmt wird und mit $A$ die Menge der Punkte des idealen Randes, die auf $a$ liegen. Es ist

$$
a=\bigcap_{n=1}^{\infty} \beta_{n i_{n},} \quad A=\bigcap_{n=1}^{\infty} B_{n i_{n}}, \quad \mathfrak{T}=\bigcap_{n=1}^{\infty} \mathfrak{B}_{n i_{n}}
$$

und 


$$
\begin{gathered}
\mathfrak{M} \cap \mathfrak{Q}=\bigcap_{n=1}^{\infty}\left(\mathfrak{R} \cap \mathfrak{B}_{n i_{n}}\right), \\
m(\mathfrak{M} \cap \mathfrak{X})=\lim _{n \rightarrow \infty} m\left(\mathfrak{R} \cap \mathfrak{B}_{n i_{n}}\right)=m(\mathfrak{M}) .
\end{gathered}
$$

Daraus ist $\mathrm{zu}$ ersehen, dass jede unteilbare Menge $M$ auf einer bestimmten idealen Randkomponente a liegt, mit Ausnahme einer Menge von harmonischem Masse Null.

Es sei $\xi(z)$ eine auf $R$ reelle messbare Funktion, so dass $\xi(\varphi(t))$ fast überall auf $|t|=1$ Winkelgrenzwerte besitzt. Wir nehmen an, dass $M$ eine unteilbare Menge von Punkten des idealen Randes ist und $\mathfrak{M}$ sei ihr Bild. Mit $\mathfrak{M}(\tau)$ bezeichnen wir die Menge jener Punkte von $\mathfrak{M}$, für welche die Winkelgrenzwerte von $\hat{\xi}(\zeta(t))$ nicht grösser als $\tau$ sind, und $m(\mathfrak{M}(\tau))$ sei ihr Lebesguesches Mass (sie ist offenbar messbar). Für jedes $\tau$ ist entweder $m(\mathfrak{M}(\tau))=0$ oder $m(\mathfrak{M}(\tau))=m(\mathfrak{M})$ denn, wenn für ein $\tau 0<m(\mathfrak{M}(\tau))<m(\mathfrak{M})$ wäre, so würden $\mathfrak{M}(\tau), \mathfrak{M}-\mathfrak{M}(\tau)$ zwei messbare automorphe Mengen sein, jede vom positiven Lebesgueschen Masse, was der Unteilbarkeit der Menge $M$ widerspricht. Da $\boldsymbol{m}(\mathfrak{M}(\tau))$ monoton ist, so gibt es ein $\tau_{0}\left(-\infty \leqq \tau_{0} \leqq+\infty\right)$, so dass $m(\mathfrak{M}(\tau))=0$ für $\tau<\tau_{0}$ und $m(\mathfrak{M}(\tau))=m(\mathfrak{M})$ für $\tau>\tau_{0}$ ist. Es ist aber für $-\infty<\tau_{0}<+\infty$

$$
\mathfrak{M}\left(\tau_{0}\right)=\bigcap_{n=1}^{\infty} \mathfrak{M}\left(\tau_{0}+\frac{1}{n}\right)
$$

und deshalb auch

$$
m\left(\mathfrak{M}\left(\tau_{0}\right)\right)=\lim _{n \rightarrow \infty} m\left(\mathfrak{M}\left(\tau_{0}+\frac{1}{n}\right)\right)=m(\mathfrak{M})
$$

Für $\tau_{0}=-\infty$ ist

$$
\mathfrak{M}\left(\tau_{0}=-\infty\right)=\bigcap_{n=1}^{\infty} \mathfrak{M}(-n)
$$

und deshalb

$$
m\left(\mathfrak{M}\left(\tau_{0}\right)\right)=\lim _{n \rightarrow \infty} m(\mathfrak{M}(-n))=m(\mathfrak{\Re})
$$

Es sei $\mathfrak{M}^{*}(\tau)$ die Menge jener Punkte von $\mathfrak{M}$, für welche $\xi(\varphi(t))$ den Winkelgrenzwert $\tau$ hat. Es ist

$$
\mathfrak{M} \mathfrak{T}^{*}\left(\tau_{0}\right)=\mathfrak{M}\left(\tau_{0}\right)-\bigcup_{n=1}^{\infty} \mathfrak{M}\left(\tau_{0}-\frac{1}{n}\right)
$$

und deshalb ist das Mass von $\mathfrak{M} \mathfrak{\}}^{*}\left(\tau_{0}\right)$ gleich dem Masse von $\mathfrak{M}$. 
Satz 2. Es sei $M$ eine unteilbare Menge von Punkten des idealen Randes einer Riemannschen Fläche $R$ und $\mathfrak{M}$ ihr Bild. Ist $\xi(z)$ eine auf $R$ reelle messbare Funktion, so dass $\xi(\varphi(t))$ fast überall auf $|t|=1$ Winkelgrenzwerte besitzt, dann sind diese Winkelgrenzwerte für fast alle $e^{i 0} \in \mathfrak{M}$ gleich einer Konstante.

Der Satz 2 ist immer anwendbar, wenn $\xi(z)=u(z)$ eine beschränkte oder halbbeschränkte harmonische Funktion ist, denn dann besitzt $u(c(t))$ nach dem Satz von Fatou fast überall Winkelgrenzwerte. Er ist auch dann anwendbar, wenn $\xi(z)=u(z)$ der reelle Teil einer analytischen Funktion $w(z)$ ist, welche eine Menge von Werten von positiver Kapazität auslässt. Dann ist nähmlich $w(\varphi(t))$ beschränktartig ([15], Seite 182) und $u(\varphi(t))$ besitzt fast überall auf $|t|=1$ Winkelgrenzwerte ([15], Seite 208). Weiter ist der Satz 2 auch dann anwendbar, wenn $\xi(z)=u(z)$, harmonisch ist und auf $R$ endliches Dirichlet Integral hat, denn es gilt

HilfSSATZ 1. Ist $u(z)$ eine auf $R$ harmonische Funktion, für die

$$
\iint_{R}|\operatorname{grad} u(z)|^{2} g\left(z, z_{0}\right) d x d y<\infty
$$

ist, so hat $u(\varphi(t))$ fast überall auf $|t|=1$ Winkelgrenzwerte.

Es sei $v(z)$ die konjugierte Funktion von $u(z)$ und $w(z)=u(z)+i v(z)$. $w(\varphi(t))$ ist eine in $|t|<1$ eindeutige und analytische Funktion. Es ist

$$
\begin{aligned}
A(r) & =\iint_{|z| \leqq r} \frac{\left|\frac{d w}{d t}\right|^{2} \rho d \rho d \psi}{\left(1+|w(\varphi(t))|^{2}\right)^{2}} \leqq \iint_{|z| \leqq r} \frac{d w^{2}}{d t} \rho d \rho d \psi \\
& =\left.\iint_{R} n(r, z) \frac{d w}{d z}\right|^{2} d x d y=\iint_{R} n(r, z)|\operatorname{grad} u(z)|^{2} d x d y
\end{aligned}
$$

wo $t=\rho e^{i \psi}$ und $n(r, z)$ die Zahl der Punkte $t$ im Kreise $|t| \leqq r$, für welche $\varphi(t)=z$ ist. Ferner ist

$$
\begin{aligned}
T(r, w) & =\int_{0}^{r} \frac{A(r) d r}{r} \leqq \int_{0}^{r} \frac{d r}{r} \iint_{R} n(r, z)|\operatorname{grad} u(z)|^{2} d x d y \\
& =\iint_{R}|\operatorname{grad} u(z)|^{2} d x d y \int_{0}^{r} \frac{n(r, z) d r}{r}
\end{aligned}
$$

Es ist aber

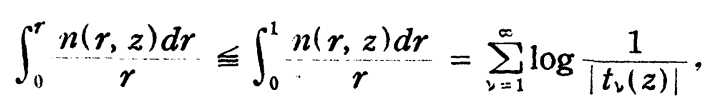


wo $t_{\nu}(z)$ die Stellen sind wo $\varphi(t)=z$ ist. Nun haben wir gesehen, dass

$$
g(\varphi(t), z)=\sum_{\nu=1}^{\infty} \log \frac{1-t \bar{t}_{\nu}(z)}{t-t_{\nu}(z)}
$$

ist, woraus man die Gleichheit

$$
\sum_{\nu=1}^{\infty} \log \frac{1}{\left|t_{\nu}(z)\right|}=g\left(z, z_{0}\right)
$$

folgert. Es ist also

$$
T(r, w) \leqq \iint_{R}|\operatorname{grad} u(z)|^{2} g\left(z, z_{0}\right) d x d y<\infty,
$$

und somit ist $w(\varphi(t))$ beschränktartig. $\quad w(\varphi(t))$ besitzt fast überall auf $|t|=1$ Winkelgrenzwerte und dasselbe gilt offenbar für $u(\varphi(t))$.

Wenn das Dirichlet-Integral von $u(z)$ endlich ist, so ist offenbar auch das Integral

$$
\iint_{R}|\operatorname{grad} u(z)|^{2} g\left(z, z_{0}\right) d x d y
$$

endlich und $u(\varphi(t))$ hat fast überall auf $|t|=1$ Winkelgrenzwerte. Da aber die Umkehrung nicht immer richtig ist, so haben wir das allgemeinere Resultat bewiesen, dass $u(\varphi(t))$ auch dann fast überall auf $|t|=1$ Winkelgrenzwerte hat, wenn das obige Integral endlich ist. Dieses Integral ist gleichzeitig für alle Pole $z_{0}$ endlich oder unendlich, wie man leicht beweisen kann.

6. Es sei $H P$ die Menge der auf $R$ harmonischen und positiven Funktionen; konstante Funktionen sind nicht ausgeschlossen. Eine Funktion $u(z) \in H P$ heisst minimal [10], wenn für jede Funktion $v(z) \in H P$, für die $v(z) \leqq u(z)$ auf $R$ ist, $v(z)=c u(z) \quad(0<c \leqq 1)$ ist. Jeder minimalen Funktion $u(z)$, welche beschränkt ist, entspricht eine unteilbare Menge und umgekehrt.

Es sei $M$ eine unteilbare Menge. Wir behaupten, dass $\omega(z, M, R)$ eine minimale Funktion ist. Denn wenn $v(z) \leqq \omega(z, M, R)$ ist, $v(z) \in H P$, so hat $v(\varphi(t))$ fast überall auf $C \mathfrak{M}$ den Winkelgrenzwert Null. Nach dem Satz 2 hat $v(\varphi(t))$ fast überall auf $\mathfrak{M}$ einen konstanten Winkelgrenzwert $c$. Es ist dann

$$
v(\varphi(t))=c \omega(\varphi(t), M, R),
$$

denn beide Funktionen sind beschränkt und haben fast überall auf $|t|=1$ denselben Winkelgrenzwert. Es ist also $v(z)=c \omega(z, M, R)$ und $\omega(z, M, R)$ ist eine minimale Funktion. 
Umgekerhrt, sei $u(z)$ eine beschränkte minimale Funktion und $\Re_{\varepsilon}$ die Menge der Punkte von $|t|=1$ wo $u(\varphi(t))$ den Winkelgrenzwert grösser als $\varepsilon(\varepsilon>0)$ hat. Wir nehmen $\varepsilon>0$ so klein, dass $\mathfrak{M}_{\varepsilon}$ positives Mass hat. Da $u(\varphi(t))$ beschränkt ist, so kann man sie durch das Poisson-Lebesguesche Integral darstellen:

$$
u(t)=\int_{0}^{2 \pi} u\left(\varphi\left(e^{i}\right)\right) P(\theta, t) d \theta
$$

wo

$$
u\left(\varphi\left(e^{i}\right)\right)=\lim _{t \rightarrow x e^{\theta i}} u(\varphi(t))
$$

ist, wo dieser Grenzwert existiert. Die Funktion

$$
v_{\varepsilon}^{*}(t)=\int_{\mathfrak{M}_{\varepsilon}} \varepsilon P(\theta, t) d \theta
$$

ist nicht grösser als $u(\varphi(t))$, denn es ist fast überall auf $|t|=1$

$$
v_{\varepsilon}^{*}\left(e^{i \theta}\right) \leqq u\left(\varphi\left(e^{i \theta}\right)\right)
$$

Da die Menge $\mathfrak{M}_{\varepsilon}$ automorph in Bezug auf $(T)$ ist, so ist, auch $v_{\varepsilon}^{*}(t)$ automorph in Bezug auf $(T)$. Daraus folgt, dass $v_{\varepsilon}(z)=v_{\varepsilon}^{*}\left(\varphi^{-1}(z)\right)$ eine auf $R$ eindeutige harmonische Funktion ist. Weiter ist $v_{\varepsilon}(z) \in H P$ und $v_{\varepsilon}(z) \leqq u(z)$ Da $u(z)$ eine minimale Funktion ist, so folgt $v_{\varepsilon}(z)=c_{\varepsilon} u(z)$. Fast überall auf $C \mathfrak{M}_{\varepsilon}$ hat also $u(\varphi(t))$ den Winkelgrenzwert Null und fast überall auf $\mathfrak{M}_{\varepsilon}$ den Winkelgrenzwert $\frac{\varepsilon}{c_{\varepsilon}}$. Daraus folgt, dass $u(\varphi(t))$ fast überall nur zwei Winkelgrenzwerte hat, und wir bezeichnen mit $\mathfrak{M}$ die Menge, wo diese Winkelgrenzwerte positiv sind. Es ist

$$
u(z)=c \omega(z, M, R) \quad\left(c=\frac{\varepsilon}{c_{\varepsilon}}\right),
$$

wo $M$ die Menge ist, die $\mathfrak{M}$ als Bild hat. Wir behaupten, dass $M$ eine unteilbare Menge ist. Im entgegengesetzten Falle kann man zwei messbare Mengen $M_{1}$, $M_{2}$ finden, so dass $M=M_{1} \cup M_{2}, M_{1} \cap M_{2}=\phi$ und $\omega\left(z, M_{\nu}, R\right)>0(\nu=1,2)$ ist. $\mathrm{Da}$

$$
\omega\left(z, M_{\nu}, R\right) \leqq \omega(z, M, R)=\frac{1}{c} u(z)
$$

ist, so ist $\omega\left(z, M_{\nu}, R\right)=c_{\nu}^{\prime} \omega(z, M, R)$. $\mathrm{Da} \omega\left(\varphi(t), M_{\nu}, R\right)$ fast überall auf $\mathfrak{M}_{\nu}$ den Winkelgrenzwert 1 hat, so folgt $c_{\nu}^{\prime}=1$ und $\omega\left(z, M_{\nu}, R\right)=\omega(z, M, R)$, was der Gleichheit 


$$
\omega(z, M, R)=\omega\left(z, M_{1}, R\right)+\omega\left(z, M_{2}, R\right)
$$

widerspricht.

SATZ 3. Das harmonische Mass einer unteilbaren Menge ist eine in der Klasse HP minimale beschränkte nicht nulle Funktion und umgekehrt, jede in der Klasse HP minimale beschränkte nicht nulle Funktion ist gleich dem harmonischen Masse einer unteilbaren Menge multipliziert mit einer beliebigen positiven Konstante.

7. Definition 2. Eine Riemannsche Fläche gehört der Klasse $U$ an, wenn sie eine unteilbare Menge auf dem idealen Rande enthält.

HilfsSATz 2. Es sei $w(z)$ eine eindeutige meromorphe Funktion auf einer Riemannschen Fläche $R \in U$. Wenn $w(\varphi(t))$ fast überall auf $|t|=1$ Winkelgrenzwerte besitzt, so ist $w(z)$ konstant.

Es sei $M$ eine unteilbare Menge des idealen Randes von $R$, $\mathfrak{M}$ ihr Bild und $w(z)=u(z)+i v(z)$. Fast überall auf $\mathfrak{M}$, haben $u(\varphi(t))$ und $v(\varphi(t))$, nach dem Satze 2, einen konstanten Winkelgrenzwert. Daraus folgt, dass auch $w(\varphi(t))$ fast überall auf $\mathfrak{M}$ einen konstanten Winkelgrenzwert hat. $\mathrm{Da} \mathfrak{M}$ positives Lebeguesches Mass hat, so folgt aus dem Satz von Priwaloff ([9], Seite 164), dass $w(\varphi(t))$ konstant ist, was $z \mathrm{u}$ beweisen war.

Daraus folgt

SATz 4. Es ist

$$
O_{H B}-O_{G} \subset U \subset O_{A C}-O_{G} \subset O_{A B}-O_{G}
$$

wo $O_{A r}$ div Klasse jener Riemannschen Fläche ist, auf welcher keine nichtkonstante meromorphe Funktion existiert, die eine Menge von Werten von positiver Kapazität auslässt.

Es ist offenbar

$$
O_{H i 3}-O_{G} \subset U \text { und } O_{A C} \subset O_{A B} \text {. }
$$

Die Beziehung $U \subseteq O_{A r}-O_{g}$ folgt unmittelbar aus dem Hilfssatz 2 und aus den Bemerkungen, die dem Satze 2 folgen. Myrberg [14] hat ein Beispiel von einer Riemannschen Fläche $R$ gebildet, die der Klasse $O_{G}$ angehört, so dass

$$
R-K \in O_{A B}
$$


ist, wo $K$ eine Kreisscheibe ist. Man kann beweisen, dass $R-K$ der Klasse $O_{A C}-O_{G}$. angehört, und da $R-K$ offenbar nicht in $U$ enthalten ist, so ist $U \subset O_{A C}-O_{G}{ }^{*}$

SATz 5. Es sei $w(z)$ eine auf $R \in U$ eindeutige meromorphe Funktion, für die das Integral

$$
\iint_{R} \frac{\left|w^{\prime}(z)\right|^{2} g\left(z, z_{0}\right) d x d y}{\left(1+|w(z)|^{2}\right)^{2}}
$$

endlich ist. Dann ist $w(z)$ konstant.

Aus dem Beweis des Hilfssatzes 1 ist $\mathrm{zu}$ ersehen, dass $w(\varphi(t))$ beschränktartig ist. $w(\varphi(t))$ hat somit fast überall auf $|t|=1$ Winkelgrenzwerte und ist laut dem Hilfssatze 2 konstant.

Bemerkung. Es ist uns nicht gelungen festzustellen ob die Klasse $U$ quasikonform invariant ist.

8. Hilfssatz 3. Es sei $R$ eine Riemannsche Fläche, für die das Integral

$$
\iint_{R} g\left(z, z_{0}\right) d \omega
$$

endlich ist; $d \omega$ ist hier der hyperbolische Flächeninhalt. Wenn eine eindeutige meromorphe Funktion $w(z)$ drei Werte nicht annimmt, dann ist das Integral

$$
\iint_{R} \frac{\left|w^{\prime}(z)\right|^{2} g\left(z, z_{0}\right) d x d y}{\left(1+|w(z)|^{2}\right)^{2}}
$$

endlich.

Es seien $w_{\nu}(\nu=1,2,3)$ die von $w(z)$ ausgelassenen Werte, und $G$ das den Punkten $w_{\nu}(\nu=1,2,3)$ auf der $w$-Ebene komplementare Gebiet. Wenn $d_{\sigma_{z}}$ bzw. $d \sigma_{w}$ das hyperbolische Mass der Fläche $R$ bzw. des Gebietes $G$ ist, so folgt nach dem Prinzip des hyperbolischen Masses $d \sigma_{w} \leqq d \sigma_{z}$. Nun ist

$$
\inf _{w \in G} \frac{\left(1+|w|^{2}\right) d \sigma_{w}}{|d w|}=\varepsilon_{G}>0
$$

Denn wäre $\varepsilon_{G}=0$, so könnte man eine Folge von Punkten $w_{n}$ finden, so dass

$$
\lim _{n \rightarrow \infty} \frac{\left(1+\left|w_{n}\right|^{2}\right) d \sigma_{w}}{|d w|}=0
$$

*) A. Mori ([13], Theorem 3) hat die Beziehung $O_{H B}-O_{G} \subseteq O_{A C}$ bewiesen. 
ist. Wir können annehmen, dass $\lim _{n \rightarrow \infty} w_{n}=w_{0}$ ist, und, da der Ausdruck

$$
\frac{\left(1+|w|^{2}\right) d \sigma_{w}}{|d \omega|}
$$

invariant bei Kugeldrehungen ist, so kann man $w_{0}=0$ nehmen. Ist $w_{0}=0 \in G$, so ist

$$
\lim _{n \rightarrow \infty} \frac{\left(1+\left|w_{n}\right|^{2}\right) d \sigma_{w}}{|d w|}=\left(\frac{d \sigma_{w}}{|d w|}\right)_{w=0}>0
$$

Man rechnet aber leicht, dass

$$
\lim _{w \rightarrow w 0,} \frac{\left(1+|w|^{2}\right) d \sigma w}{|d w|}=\infty \quad(\nu=1,2,3)
$$

ist, so dass die Voraussetzung $\varepsilon_{G}=0$ jedenfalls $z u$ einem Widerspruch führt. Daraus folgert man

$$
\frac{|d w|}{1+|\omega|^{2}} \leqq \frac{1}{\varepsilon_{G}} d \sigma_{w} \leqq \frac{1}{\varepsilon_{F}} d \sigma_{z}
$$

und

$$
\iint_{R} \frac{\left|w^{\prime}(z)\right|^{2} g\left(z, z_{0}\right) d x d y}{\left(1+|w(z)|^{2}\right)^{2}} \leqq \frac{1}{\varepsilon_{(;}^{2}} \iint_{R} g\left(z, z_{0}\right) d \omega<\infty
$$

was $\mathrm{zu}$ beweisen war.

Aus dem Hilfssatze 3 und aus dem Satze 5 folgt

Satz 6. Es sei $R \in U$ eine Riemannsche Fläche, für die

$$
\iint_{R} g\left(z, z_{0}\right) d \omega<\infty
$$

ist, wo dw der hyperbolische Flächeninhalt ist. Ist $w(z)$ eine auf $R$ meromorphe Funktion, welche drei Werte auslässt, so ist $w(z)$ konstant.

\section{Extremisierung}

9. Es sei $\Delta$ eine offene Menge auf $R$, deren Rand $\partial \Delta$ aus höchstens abzählbar vielen analytischen Jordanbogen besteht. Wir nehmen an, dass nur endlich viele von ihnen einen nichtleeren Durchschnitt mit einem beliebigen Kompakt haben. In dieser Arbeit werden wir stets einen solchen Rand "analytischen Rand" benennen. Es sei $\left\{R_{n}\right\}$ eine Ausschöpfung der Fläche $R$, wo der Rand $\partial R_{n}$ von $R_{n}$ aus endlich vielen analytischen Jordankurven besteht.

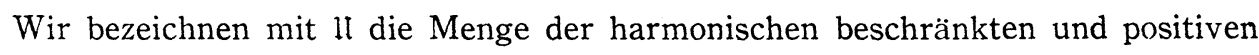


Funktionen auf $R$ und mit $\mathfrak{u}^{\prime}$ die Menge der harmonischen beschränkten und positiven Funktionen auf $\Delta$, stetig auf $\bar{\Delta}$, die auf $\partial \Delta$ verschwinden. Die Mengen $\mathfrak{u}$ und $\mathfrak{u}^{\prime}$ sind Halbmodule. Wir werden zwei natürliche lineare Abbildungen zwischen diese Mengen einführen, die nichts anderes als die für unsere $Z$ wecke passend abgeänderte Extremisation und Inextremisation von Kuramochi sind [5].

Es seien $u \in \mathfrak{U}$ und $u^{\prime} \in \mathfrak{u}^{\prime}$. Wir bezeichnen mit $E_{n} u^{\prime}$ die in $R_{n}$ harmonische und auf $\bar{R}_{n}$ stetige Funktion, für welche

$$
E_{n} u^{\prime}=\left\{\begin{array}{lll}
0 & \text { auf } & \partial R_{n} \cap C \Delta \\
u^{\prime} & \text { auf } & \partial R_{n} \cap \Delta
\end{array}\right.
$$

gilt, und mit $I_{n} u$ die in jeder Komponente von $R_{n} \cap \Delta$ harmonische und auf $\overline{R_{n} \cap \Delta}$ stetige Funktion (mit Ausnahme endlich vieler Punkte), für welche

$$
I_{n} u=\left\{\begin{array}{lll}
0 & \text { auf } & \partial \Delta \cap R_{n} \\
u & \text { auf } & \partial R_{n} \cap \Delta
\end{array}\right.
$$

ist. Die Operatoren $E_{n}$ und $I_{n}$ haben folgende Eingenschaften:

$$
\begin{array}{ll}
E_{n} u^{\prime} \leqq \sup u^{\prime}, & I_{n} u \geqslant 0 \\
E_{n} u^{\prime} \geqq u^{\prime}, & I_{n} u \leqq u
\end{array}
$$

Daraus folgt

$$
E_{n} u^{\prime} \leqq E_{n+1} u{ }^{\prime}, \quad I_{n} u \geqslant I_{n-1} u
$$

Für eine positive Zahl $c$ ist

$$
E_{n} c u^{\prime}=c E_{n} u^{\prime}, \quad I_{n} c u=c I_{n} u
$$

Für $u_{1}^{\prime}, u_{2}^{\prime} \in \mathfrak{u}^{\prime}$ und $u_{1}, u_{2} \in \mathfrak{U}$ ist

$$
E_{n}\left(u_{1}^{\prime}+u_{2}^{\prime}\right)=E_{n} u_{1}^{\prime}+E_{n} u_{2}^{\prime}, \quad I_{n}\left(u_{1}+u_{2}\right)=I_{n} u_{1}+I_{n} u_{2}
$$

Aus $u_{1}^{\prime} \leqq u_{2}^{\prime}$ und $u_{1} \leqq u_{2}$ folgt

$$
E_{n} u_{1}^{\prime} \leqq E_{n} u_{2}^{\prime}, \quad I_{n} u_{1} \leqq I_{n} u_{2}
$$

Da die Folgen $\left\{E_{n} u^{\prime}\right\},\left\{I_{n} u\right\}$ monoton sind, so konvergieren sie nach dem Harnackschen Prinzip gleichmässig gegen harmonische Funktionen

$$
E u^{\prime}=\lim _{n \rightarrow \infty} E_{n} u^{\prime}, \quad I u=\lim _{n \rightarrow \infty} I_{n} u .
$$

Die Funktionen $E u^{\prime}$ und $I u$ sind harmonisch auf $R$ bzw. auf $A$, und haben folgende Eigenschaften 


$$
u^{\prime} \leqq E_{n} u^{\prime} \leqq E u^{\prime} \leqq \sup u^{\prime}, \quad u \geqq I_{n} u \geqq I u \geqq 0 .
$$

Für eine positive Zahl $c$ ist

$$
E c u^{\prime}=c E u^{\prime}, \quad I c u=c I u
$$

Für $u_{1}^{\prime}, u_{2}^{\prime} \in \mathfrak{H}^{\prime}$ und $u_{1}, u_{2} \in \mathfrak{u}$ ist

$$
E\left(u_{1}^{\prime}+u_{2}^{\prime}\right)=E u_{1}^{\prime}+E u_{2}^{\prime}, \quad I\left(u_{1}+u_{2}\right)=I u_{1}+I u_{2}
$$

Aus $u_{1}^{\prime} \leqq u_{2}^{\prime}$ und $u_{1} \leqq u_{2}$ folgt

$$
E u_{1}^{\prime} \leqq E u_{2}^{\prime}, \quad I u_{1} \leqq I u_{2}
$$

Es sei $u^{\prime}, \in \mathfrak{u}^{\prime}$ und $u^{\prime}=\sum_{\nu=1}^{\infty} u_{\nu}^{\prime} \in \mathfrak{u}^{\prime} . \quad$ Dann ist

$$
E_{n} u^{\prime}=\sum_{\nu=1}^{\infty} E_{n} u_{\nu}^{\prime}
$$

und daraus

$$
E u^{\prime} \geq \sum_{\nu=1}^{\infty} E_{n} u_{\nu}^{\prime} \geqslant \sum_{\nu=1}^{\nu} E_{n} u_{\nu}^{\prime}
$$

Für $n \rightarrow \infty$ folgt $E u^{\prime} \geqq \sum_{\nu=1}^{N} E u_{\imath}^{\prime}$ und für $N \rightarrow \infty$ erhalten wir

$$
E u^{\prime} \geqslant \sum_{\nu=1}^{\infty} E u_{\nu}^{\prime}
$$

Es ist aber

$$
\sum_{\nu=1}^{\infty} E u_{\nu}^{\prime} \geq \sum_{\nu=1}^{\infty} E_{n} u_{\nu}^{\prime}=E_{n} u^{\prime}
$$

und also für $n \rightarrow \infty, \sum_{\nu=1}^{\infty} E u_{\nu}^{\prime} \geqslant E u^{\prime}$, woraus

$$
E u^{\prime}=\sum_{\nu=1}^{\infty} E u^{\prime}
$$

folgt. Es sei weiter $u_{\nu} \in \mathfrak{U}$ und $u=\sum_{\nu=1}^{\infty} u_{\nu} \in \mathfrak{u}$. Dann ist

$$
I_{n} u=\sum_{v=1}^{\infty} I_{n} u
$$

Daraus folgt $I_{n} u \geqslant \sum_{\nu=1}^{\infty} I u_{\nu}$ und also ist

$$
I u \geqslant \sum_{\nu=1}^{\infty} I u
$$

Es sei $z \in R_{n}$ und $\nu(\varepsilon, z)$ so gross, dass 


$$
\sum_{\nu=1+\nu(\varepsilon, z)}^{\infty} I_{n} u_{\nu}(z)<\varepsilon
$$

ist. Dann ist

$$
I u(z) \leqq \sum_{\nu=1}^{\nu(\varepsilon, z)} I_{n} u_{i}(z)+\varepsilon .
$$

Für $n \rightarrow \infty$ ist also

$$
I u(z) \leqq \sum_{\nu=1}^{\nu(\xi, z)} I u_{\nu}(z)+\varepsilon \leqq \sum_{\nu=1}^{\infty} I u_{\nu}(z)+\varepsilon
$$

und da $\varepsilon$ beliebig klein war, folgt $I u=\sum_{\nu=1}^{\infty} I u_{\nu}$.

Es ist also

$$
E \sum_{\nu=1}^{\infty} u_{\nu}^{\prime}=\sum_{\nu=1}^{\infty} E u_{\nu}^{\prime}, \quad I \sum_{\nu=1}^{\infty} u_{\nu}=\sum_{\nu=1}^{\infty} I u_{\nu}
$$

Aus der Monotonie der Folgen $E_{n} u^{\prime}, I_{n} u$ folgt, dass $E u^{\prime}$ bzw. Iu unabhängig von der gewählten Ausschöpfung sind und Funktionen aus $\mathfrak{u} b z w$. $\mathfrak{u}^{\prime}$ darstellen. $E u^{\prime}$ und $I u$ sind Linearoperatoren zwischen diesen Halbmodulen $\mathfrak{u}$ und $\mathfrak{l}^{\prime}$. Wir werden die Operationen $E$ bzw. $I$ die Extremisation bzw. Inextrmisation in Bezug auf das Paar $(\Delta, R)$ benennen.

Der Funktion $E u^{\prime}$ kann man den Operator $I$ anwenden:

$$
I E u^{\prime}=\lim _{n \rightarrow \infty} I_{n} E u^{\prime}
$$

Aus $E u^{\prime} \geqq u^{\prime}$ folgt in $R_{n} \cap \Delta$

$$
I_{n} E u^{\prime} \geqslant u^{\prime}
$$

und also $I E u^{\prime} \geqq u^{\prime}$ überall in $\Delta$. Andererseits ist in $R_{n} \cap \Delta$

$$
E_{n} u^{\prime}+I_{n} E u^{\prime} \leqq u^{\prime}+E u^{\prime}
$$

da auf $\partial R_{n} \cap \Delta, E E_{n} u^{\prime}=u^{\prime}$ und $I_{n} E u^{\prime}=E u^{\prime}$ und auf $\partial \Delta \cap R_{n}, I_{n} E u^{\prime}=u^{\prime}=0$ und $E_{n} u^{\prime}$ im allgemeinen nicht grösser als $E u^{\prime}$ ist. Für $n \rightarrow \infty$ ist in $\Delta$

$$
E u^{\prime}+I E u^{\prime} \leqq u^{\prime}+E u^{\prime}
$$

oder $I E u^{\prime} \leqq u^{\prime}$, woraus $I E u^{\prime}=u^{\prime}$ folgt.

Wir werden den Operator $E$ der Funktion $I u$ anwenden:

$$
E I u=\lim _{n \rightarrow \infty} E_{n} I u .
$$

Aus $I u \leqq u$ folgt $E_{n} I u \leqq u$ und also 


\section{$E I u \leqq u$.}

Es seien $u_{1}, u_{2} \in \mathfrak{u}$, Aus $u_{1} \leqq u_{2}$ folgt auf $R_{n} \cap \Delta$

$$
u_{1}-I_{n} u_{1} \leqq u_{2}-I_{n} u_{2},
$$

denn auf $\partial \Delta \cap R_{n}$ ist $I_{n} u_{1}=I_{n} u_{2}=0$ und auf $\partial R_{n} \cap \Delta$ sind beide Seiten der Ungleichung null. Für $n \rightarrow \infty$ folgt auf $\Delta$

$$
u_{1}-I u_{1} \leq u_{2}-I u_{3} \text {. }
$$

Weiter ist, auf $R_{n}, u_{1}-E_{n} I u_{1} \leqq u_{2}-E_{n} I u_{2}$, denn auf $\partial R_{n} \cap C \Delta$ ist $E_{n} I u_{1}=E_{n} I u_{2}$ $=0$ und auf $\partial R_{n} \cap \Delta$ ist

$$
u_{1}-E_{n} I u_{1}=u_{1}-I u_{1} \leqq u_{2}-I u_{2}=u_{2}-E_{n} I u_{2} .
$$

Für $n \rightarrow \infty$ folgt dann auf $R$

$$
u_{1}-E I u_{1} \leqq u_{2}-E I u_{2} .
$$

Falls $\Delta=R-R_{0}$ ist, so ist $u$ - Iu -die normierte Lösung von Nevanlinna ([16], Seite 320) und $I 1$ ist das harmonische Mass des idealen Randes.

10.*) Der Operator $I$ führt die ganze Menge $\mathfrak{U}$ auf die ganze Menge $\mathfrak{u}$ 'über, denn für jedes $u^{\prime} \in \mathfrak{u}^{\prime}$ existiert ein $u \in \mathfrak{u}, u=E u^{\prime}$, für welches $u^{\prime}=I u$ ist. Wir fragen nach dem Kern dieser Abbildung; das ist die Teilmenge $\mathfrak{u}_{0}$ von $\mathfrak{u}$ die durch $I$ ins 0 -Element übergeführt wird. Gleichzeitig untersuchen wir eine andere Teilmenge $\mathfrak{u}_{1}$ von $\mathfrak{u}$, die das Bild von $\mathfrak{u}^{\prime}$ durch $E$ ist. $\mathfrak{u}_{0}$ und $\mathfrak{u}_{1}$ sind auch Halbmodulen. $E$ ist eine isomorphe Abbildung von $\mathfrak{u}^{\prime}$ auf $\mathfrak{u}_{1}$, denn aus $E u_{1}^{\prime}=E u_{2}^{\prime}$ folgt $u_{1}^{\prime}=I E u_{1}^{\prime}=I E u_{2}^{\prime}=u_{2}^{\prime}$. Auch $I$ ist eine isomorphe Abbildung von $\mathfrak{l}_{1}$ auf $\mathfrak{u}^{\prime}$ und $z$ war die Umkehrung von $E$, denn es seien $u_{1}, u_{2}$ zwei Funktionen aus $\mathfrak{u}_{1}$ für die $I u_{1}=I u_{2}$ gilt. Da $\mathfrak{u}_{1}$ das Bild von $\mathfrak{u}^{\prime}$ durch $E$ ist, so kann man zwei Funktionen $u_{1}^{\prime}, u_{2}^{\prime}$ aus $\mathfrak{u}^{\prime}$ finden, für die $u_{2}=E u_{v}^{\prime}(\nu=1,2)$ ist. Es ist aber

$$
u_{1}^{\prime}=I E u_{1}^{\prime}=I u_{1}=I u_{2}=I E u_{2}^{\prime}=u_{2}^{\prime},
$$

woraus $u_{1}=u_{2}$ folgt. Weiter ist für $u \in \mathfrak{u}_{1}$

$$
E I u=u .
$$

Denn es ist $I E I u=I u$, woraus sich die Behauptung ergiht. Für ein beliebiges

*) Inzwischen haben wir die Arbeit von M. Heins [3] gelesen, wo auch die Extremisierung und Inextremisierung eingeführt wurde, und wo der grösste Teil der Ergebnisse dieses Absatzes zu finden sind. 
$u \in \mathfrak{U}$ ist $u_{1}=E I u \in \mathfrak{H}_{1} . \quad$ Da $u_{1} \leqq u$ ist, so ist $u_{0}=u-u_{1} \in \mathfrak{U}$ und da

$$
I u=I u_{0}+I u_{1}
$$

und

$$
I u_{1}=I E I u=I u
$$

ist, so folgt $I u_{0}=0$. Es ist also $u_{0} \in \mathfrak{u}_{0}$. Jede Funktion $u \in \mathfrak{u}$ erhält damit eine Zerlegung $u=u_{0}+u_{1}$, wo

$$
u_{0}=u-E I u \in \mathfrak{U}_{0}, \quad u_{1}=E I u \in \mathfrak{H}_{1}
$$

ist. Es ist klar, dass diese Zerlegung eindeutig durch $u$ bestimmt ist. Denn aus $u=v_{0}+v_{1}\left(v_{0} \in \mathfrak{H}_{0}, v_{1} \in \mathfrak{H}_{1}\right)$ folgt

$$
I u=I u_{1}=I v_{1},
$$

woraus $u_{1}=v_{1}$ folgt. Die Funktionen $u_{0}$ und $u_{1}$ besitzen folgende Eigenschaften

$$
u_{0}=\sup v_{0} \quad \mathfrak{H}_{0} \ni v_{0} \leqq u, \quad u_{1}=\sup v_{1} \quad \mathfrak{H}_{1} \ni v_{1} \leqq u .
$$

Denn aus $\mathfrak{l}_{0} \ni v_{0} \leqq u, \mathfrak{l}_{1} \ni v_{1} \leqq u$. folgt

$$
\begin{aligned}
& v_{0}=v_{0}-E I v_{0} \leqq u-E I u=u_{0}, \\
& v_{1}=E I v_{1} \leqq E I u=u_{1} .
\end{aligned}
$$

Es ist also für $u \leqq v$

$$
u_{0} \leqq v_{0}, \quad u_{1} \leqq v_{1}
$$

Zwischen den Elementen von $\mathfrak{u}_{0}$ und $\mathfrak{u}_{1}$ besteht keine Ordnungsbeziehung: aus $u_{0} \in \mathfrak{u}_{0}, u_{1} \in \mathfrak{H}_{1}$ und $u_{0} \leqq u_{1}$ folgt $u_{0}=0$ und ähnlicherweise, wenn $u_{1} \leqq u_{0}$ ist, so ist auch $u_{1}=0$.

Es entsteht die Frage, unter welchen Bedingungen $\mathfrak{l}$ und $\mathfrak{u}^{\prime}$ isomorph sind; diese Frage ist gleichbedeutend mit der Frage, wann $\mathfrak{U}_{0}$ auf dem 0-Element sich reduziert. Wir werden beweisen, dass

$$
E I 1 \equiv 1
$$

die notwendige und hinreichende Bedingung dafür ist, dass $\mathfrak{l}$ und $\mathfrak{l}^{\prime}$ iscmorph seien. Es ist notwendig und hireichend, dass

$$
u_{0}=u-E I u=0
$$

für jedes $u \in \mathfrak{l}$ ist. Wenn man also die Funktion $u(z) \equiv 1 \in \mathfrak{l}$ nimmt, so ist $E I 1 \equiv 1$ eine notwendige Bebingung. Für ein beliebiges $u \in \mathfrak{l}$ sei $c=\sup u$. 
Dann ist $c-u \in \mathfrak{u}$ und $E I(c-u) \leqq c-u$ oder $c E I 1-E I u \leqq c-u$. Ist $E I 1=1$, so folgt $u \leqq E I u$ und aus der allgemeinen Ungleichung $E I u \leqq u$ folgt $u=E I u$, was $z \mathrm{u}$ beweisen war.

Die Operatoren $E$ und I haben noch eine wichtige Eigenschaft: sie führen minimale Elemente in minimale Elemente über. Es sei $u^{\prime}$ eine minimale Funktion der Menge $\mathfrak{u}^{\prime}$ und $u \in \mathfrak{u}$

$$
u \leqq E u^{\prime}
$$

Daraus folgt, dass in der Zerlegung von $u, u=u_{0}+u_{1}$, die Komponente $u_{0}$ verschwindet und $u=u_{1} \in \mathfrak{u}_{1}$. Ferner ist

$$
I u \leqq I E u^{\prime}=u^{\prime},
$$

woraus $I u=c u^{\prime}$ und $u=E I u=c E u^{\prime}$ folgt. Dass heisst, dass $E u^{\prime}$ minimal ist. Es sei jetzt $u$ eine minimale Funktion in der Klasse $\mathfrak{u}$. Aus $u^{\prime} \in \mathfrak{u}^{\prime}$ und $u^{\prime} \leqq I u$ folgt dann

$$
E u^{\prime} \leqq E I u \leqq u,
$$

und da $u$ minimal ist, ist $E u^{\prime}=c u$ und $u^{\prime}=I E u^{\prime}=c I u$. I $u$ ist somit minimal.

11. Die Funktion $I 1$ besitzt eine Extremaleigenschaft: ist $\mathfrak{u}^{\prime} \in \mathfrak{u}^{\prime}$ kleiner als eins, so ist $u^{\prime} \leqq I 1$. Denn in $R_{n} \cap \Delta$ ist $u^{\prime} \leqq I_{n} 1$ und für $n \rightarrow \infty$ ist $u^{\prime} \leqq I 1$.

Die Funktion $E I 1=E I 1(z)$ ist harmonisch auf $R$ lind nicht grösser als 1 . Wir werden mit $\mathfrak{M}_{0}$, bzw. $\mathfrak{N}_{\varepsilon}$, bzw. $\mathfrak{M}_{1}$ die Menge der Punkte $e^{i 0}$ aus $\tilde{\jmath}$ bezeichnen, wo $\operatorname{EI} 1\left(\varphi\left(e^{i \theta}\right)\right)=0$ bzw. $\varepsilon \leqq E I 1\left(\varphi\left(e^{i \theta}\right)\right) \leqq 1-\varepsilon$ bzw. $E I 1\left(\varphi\left(e^{\theta i}\right)\right)=1$ ist, und mit $M_{0}$, bzw. $N_{\varepsilon}$, bzw. $M_{1}$ die Menge der Punkte des idealen Randes, deren Bild $\mathfrak{M}_{9}$, bzw. $\mathfrak{R}_{s}$, bzw. $\mathfrak{M}_{1}$ ist. Es ist $\omega\left(z, N_{s}, R\right) \leqq 1$. Daraus folgt $E I \omega\left(z, N_{\varepsilon}, R\right) \leqq E I 1(z)$ und

$$
E I \omega\left(\varphi\left(e^{0 i}\right), N_{\varepsilon}, R\right) \leqq E I 1\left(\varphi\left(e^{i \theta}\right)\right) .
$$

Für fast alle $e^{i 0} \in \mathfrak{N}_{\varepsilon}$ ist also

$$
\operatorname{EI\omega }\left(\varphi\left(e^{i \theta}\right), N_{s}, R\right) \leqq 1-\varepsilon
$$

und für fast alle $e^{i \theta} \in C \mathfrak{N}_{\varepsilon}$ ist

$$
E I \omega\left(\varphi\left(e^{i \theta}\right), N_{s}, R\right) \leqq \omega\left(\varphi\left(e^{i \theta}\right), N_{\varepsilon}, R\right)=0 .
$$

Daher folgt

$$
E I \omega\left(z, N_{\varepsilon}, R\right) \leqq(1-\varepsilon) \omega\left(z, N_{\varepsilon}, R\right)
$$


und

$$
E I \omega\left(z, N_{\S}, R\right)=\operatorname{EIEI\omega }\left(z, N_{\varepsilon}, R\right) \leqq(1-\varepsilon) E I \omega\left(z, N_{\S}, R\right)
$$

was als Folge $E I \omega\left(z, N_{s}, R\right)=0$ und $\omega\left(z, N_{s}, R\right) \in \mathfrak{H}_{0}$ hat. Es ist aber

$$
\varepsilon \omega\left(z, N_{\S}, R\right) \leqq E I 1(z)
$$

und da $E I 1(z) \in \mathfrak{U}_{1}$, so folgt nach 10 ., $\omega\left(z, N_{s}, R\right)=0$. Die Menge $\eta_{s}$ hat also nulles Lebesguesches Mass und, da das für jedes $\varepsilon>0$ gilt, so folgt, dass

$$
m\left(\mathfrak{M}_{0}\right)+m\left(\mathfrak{M}_{1}\right)=2 \pi
$$

ist. Ausserhalb der Menge $\mathfrak{M}_{1}$ hat $E I 1(\varphi(t))$ fast überall den Winkelgrenzwert Null und

$$
\begin{aligned}
& E I 1(z)=\omega\left(z, M_{1}, R\right) \in \mathfrak{U}_{1}, \\
& 1-E I 1(z)=\omega\left(z, M_{0}, R\right) \in \mathfrak{H}_{0} .
\end{aligned}
$$

Die Beziehung

$$
1=\omega\left(z, M_{0}, R\right)+\omega\left(z, M_{1}, R\right)
$$

stellt gerade für die Funktion $u(z) \equiv 1$ die Zerlegung dar, die wir in 10 . eingeführt haben. Die notwendige und hinreichende Bedingung dafür, dass II und $\mathfrak{u}^{\prime}$ isomorph sein sollen ist also, dass

$$
\omega\left(z, M_{0}, R\right)=0
$$

sein soll.

Wir werden jetzt den Ausdruck der Komponenten $u_{0}$ und $u_{1}$ geben die in der Zerlegung der Funktion $u \in \mathfrak{u}$ eingetreten sind. Aus $u \leqq c=\sup u$ folgt

$$
u_{0} \leqq c \omega\left(z, M_{0}, R\right), \quad u_{1} \leqq c \omega\left(z, M_{1}, R\right) .
$$

Also hat $u_{0}(\varphi(t))$ bzw. $u_{1}(\varphi(t))$ überall auf $\mathfrak{M}_{1}$ bzw. $\mathfrak{M}_{0}$ nulle Winkelgrenzwerte und aus $u=u_{0}+u_{1}$ folgt

$$
\begin{aligned}
& u_{0}(\varphi(t))=\int_{\mathfrak{M}_{0}} u\left(\varphi\left(e^{i 0}\right)\right) P(\theta, t) d \theta \\
& u_{1}(\varphi(t))=\int_{\mathfrak{M}_{1}} u\left(\varphi\left(e^{i 0}\right)\right) P(\theta, t) d \theta
\end{aligned}
$$

Also haben EIu(

Es sei $R^{\prime}$ eine Komponente der offenen Menge 4 . Sie ist selbst eine Riemannsche Fläche und wir können, genau wie für $R$, die Punkte des idealen Randes von $R^{\prime}$ einführen. Sei $z=\varphi^{\prime}\left(t^{\prime}\right)$ die eineindeutige und konforme 
Abbildung des Kreises $\left|t^{\prime}\right|<1$ auf die universelle Überlagerungsfläche von $R^{\prime}$ und $\hat{\gamma}^{\prime}$ sei die Menge der Punkte $e^{i 0^{\prime}}$, wo $g^{\prime}\left(\varphi^{\prime}\left(t^{\prime}\right), z_{0}\right)$ den Winkelgrenzwert Null hat, wo $g^{\prime}\left(z, z_{0}\right)$ die Greensche Funktion von $R^{\prime}$ ist. Die Punkte des idealen Randes von $R^{\prime}$ werden die Äquivalenzklassen von $\hat{F}^{\prime}$ in Bezug auf die Deckbewegungsgruppe $\left(T^{\prime}\right)$ sein. Wir werden mit $\mathfrak{D}_{0}^{\prime}$ die Menge jener Punkte $e^{i \theta^{\prime}}$ bezeichnen, die dem relativen Rande $\partial R^{\prime}$ von $R^{\prime}$ entspricht; das ist gleichbedeutend mit der Existenz des Limes $\lim _{t^{\prime} \rightarrow e^{i \theta}} \varphi^{\prime}\left(t^{\prime}\right)$. $\mathfrak{W}_{0}^{\prime}$ und $\widetilde{F}^{\prime}-\mathfrak{W}_{0}^{\prime}=\mathfrak{W}_{1}^{\prime}$ sind automorph in Bezug auf $\left(T^{\prime}\right)$. Deshalb entsprechen ihnen zwei Mengen von Punkten des idealen Randes von $R^{\prime}, M_{0}^{\prime}$ und $M_{1}^{\prime}$. Das harmonische Mass der Menge $M_{1}^{\prime}, \omega\left(z, M_{1}^{\prime}, R^{\prime}\right)$ gehört der Menge $\mathfrak{u}^{\prime}$ an und ist kleiner als 1 . Wegen der obenerwähnten Extremaleigenschaft der Funktion $I 1(z)$ ist auf $R^{\prime}$

$$
\omega\left(z, M_{1}^{\prime}, R^{\prime}\right) \leqq I 1(z) \leqq 1 .
$$

Daraus folgt, dass $I 1\left(\varphi^{\prime}\left(t^{\prime}\right)\right)$ fast überall auf $\mathfrak{M}_{1}^{\prime}$ den Winkelgrenzwert 1 hat: da $I 1\left(\varphi^{\prime}\left(e^{i \theta^{\prime}}\right)\right)=0$ für $e^{i \theta^{\prime}} \in \mathbb{M}_{0}^{\prime}$ ist, so folgt auf $R^{\prime}$

$$
\omega\left(z, M_{1}^{\prime}, R^{\prime}\right)=I 1(z) \text {. }
$$

Es sei jetzt $\Delta=\bigcup_{\nu} R_{\nu}^{\prime}$, wo $\left\{R_{\nu}^{\prime}\right\}$ die Komponente der offenen Menge $\Delta$ sind. Für $u \in \mathfrak{U}$ werden wir mit $I_{(\nu)} u$ die Funktionen

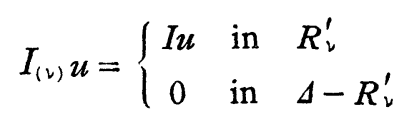

bezeichnen. Der Operator $I_{(\nu)} u$ ist nichts anderes als die Inextremisation von $u$ in Bezug auf das Paar $\left(R_{\nu}^{\prime}, R\right)$. Die Funktion $E I_{(v)} 1$ hat fast dieselbe Eigenschaften wie $E I 1$. Insbesondere hat $E I_{(\nu)} 1(\varphi(t))$ überall auf einer Menge $\mathfrak{R}_{(\nu) 1}$ den Winkelgrenzwert 1 und fast überall auf dem Komplement von $\mathfrak{M}_{(v) 1}$ den Winkelgrenzwert 0 . Wenn $M_{(v) 1}$ die Menge, deren Bild $\mathfrak{M}_{(v) 1}$ ist, bezeichnet, so ist

$$
E I_{(\nu)} 1(z)=\omega\left(z, M_{(\nu ; 1}, R\right) .
$$

Aus $I 1=\sum_{\nu} I_{(\nu)} 1$ folgt (siehe 9)

$$
\omega\left(z, M_{1}, R\right)=E I 1(z)=\sum_{\nu} E I_{(\nu)} 1(z)=\sum_{\nu} \omega\left(z, M_{(\nu) 1}, R\right),
$$

woraus $\mathrm{zu}$ ersehen ist, dass die Mengen $M_{(\llcorner) 1}$ paarweise punktfremd sind. Es ist also 


$$
\omega\left(z, M_{1}, R\right)=\sum \omega\left(z, M_{(\nu) 1}, R\right)=\omega\left(z, \cup M_{(v) 1}, R\right) .
$$

Weiter folgt

$$
\cup M_{(\cdot) 1} \cong M_{1}
$$

und, dass das harmonische Mass der Menge

$$
M_{1}-\cup M_{(\llcorner) 1}
$$

null ist.

Es sei jetzt $u \in \mathfrak{U}$ und $c=\sup u$. Auf $R_{n} \cap \Delta$ ist $c I_{n} 1+u \leqq I_{n} u+c$ und daher ist

$$
c I 1+u \leqq I u+c .
$$

Wenn $R^{\prime}$ eine Komponente von $\Delta$ ist, so folgt aus dieser Ungleichung und aus $I u \leqq u$ dass $I u\left(\varphi^{\prime}\left(t^{\prime}\right)\right)$ und $u\left(\varphi^{\prime}\left(t^{\prime}\right)\right)$ fast überall auf $\mathfrak{M}_{1}^{\prime}$ dieselben Winkelgrenzwerte haben. Aus $I E I u=I u$ folgt, dass $I u\left(\varphi^{\prime}\left(t^{\prime}\right)\right)$ und $E I u\left(\varphi^{\prime}\left(t^{\prime}\right)\right)$ fast überall auf $\mathfrak{M}_{1}^{\prime}$ dieselben Winkelgrenzwerte haben. Also haben $u\left(\varphi^{\prime}\left(t^{\prime}\right)\right), I u\left(\varphi^{\prime}\left(t^{\prime}\right)\right)$ und $\operatorname{EIu}\left(\varphi^{\prime}\left(t^{\prime}\right)\right)$ fast überall auf $\mathfrak{M}_{1}^{\prime}$ dieselben Winkelgrenzwerte und es ist

$$
\operatorname{Iu}\left(\varphi^{\prime}\left(t^{\prime}\right)\right)=\int_{\mathfrak{M}_{1}^{\prime}} u\left(\varphi^{\prime}\left(e^{i \theta^{\prime}}\right)\right) P\left(\theta^{\prime}, t^{\prime}\right) d \theta^{\prime}
$$

12. In diesem Absatz nehmen wir an, dass $\Delta=R^{\prime}$ zusammenhängend ist. Es sei $M$ eine messbare Menge des idealen Randes von $R$. Wir bezeichnen mit $\mathfrak{M}^{\prime}$ bzw. $\mathfrak{M}_{\S}^{\prime}$ die Menge jener $e^{i 0^{\prime}} \in \widetilde{\mho}^{\prime}$, für die

$$
I \omega\left(\varphi^{\prime}\left(e^{i \theta^{\prime}}\right), M, R\right)=1 \quad \text { bzw. } \quad \varepsilon \leqq I \omega\left(\varphi^{\prime}\left(e^{i \theta^{\prime}}\right), M, R\right) \leqq 1-\varepsilon
$$

ist, und $M^{\prime}$ bzw. $M_{\varepsilon}^{\prime}$ sei die Menge von Punkten des idealen Randes der Fläche $R^{\prime}$, welche $\mathfrak{M}^{\prime}$ bzw. $\mathfrak{M}_{\varepsilon}^{\prime}$ als Bild hat. Es ist offenbar

$$
\begin{aligned}
& \varepsilon \omega\left(z, M_{\varepsilon}^{\prime}, R^{\prime}\right) \leqq I \omega(z, M, R), \\
& I \omega(z, M, R)+\varepsilon \omega\left(z, M_{\varepsilon}^{\prime}, R^{\prime}\right) \leqq I 1(z) .
\end{aligned}
$$

Da $I \omega(z, M, R)$ Null auf $\partial R^{\prime}$ ist, so ist auch $\omega\left(z, M_{\varepsilon}^{\prime}, R^{\prime}\right)$ Null auf $\partial R^{\prime}$ und gehört somit der Klasse $\mathfrak{u}^{\prime}$ an. Daraus folgt

$$
\begin{aligned}
& \varepsilon E \omega\left(z, M_{\varepsilon}^{\prime}, R^{\prime}\right) \leqq E I \omega(z, M, R), \\
& E I \omega(z, M, R)+\varepsilon E \omega\left(z, M_{\varepsilon}^{\prime}, R^{\prime}\right) \leqq E I 1(z) .
\end{aligned}
$$

Nun sieht man aus der Darstellung des Operators $E I$ durch das PoissonLebesguesche Integral, dass $E I \omega(z, M, R)=\omega\left(z, M \cap M_{1}, R\right)$ ist. Aus der 
ersten Ungleichung folgt, dass $E \omega\left(\varphi(t), M_{\S}^{\prime}, R^{\prime}\right)$ fast überall auf $C\left(\mathfrak{M} \cap \mathfrak{R}_{1}\right)$ den Winkelgrenzwert Null hat und aus der zweiten, dass sie auch auf $\mathfrak{M} \cap \mathfrak{R}_{1}$ fast überall den Winkelgrenzwert Null hat. Das heisst $E \omega\left(z, M_{\varepsilon}^{\prime}, R^{\prime}\right)=0$ oder $\omega\left(z, M_{\varepsilon}^{\prime}, R^{\prime}\right)=I E \omega\left(z, M_{\varepsilon}^{\prime}, R^{\prime}\right)=0$ und, da $\varepsilon$ beliebig war, so folgert man, dass $I \omega\left(\iota^{\prime}\left(t^{\prime}\right), M, R\right)$ fast überall auf $C \mathfrak{P}^{\prime}$ den Winkelgrenzwert Null hat. Wir werden

$$
M^{\prime}=I^{*} M
$$

stellen. Es ist $I \omega(z, M, R)=\omega\left(z, I^{*} M, R\right)$.

Die Abbildung $I^{*}$ führt die messbaren Mengen des idealen Randes von $R$ in messbare Mengen des idealen Randes von $R^{\prime}$ über und zwar in Teilmengen der Menge $M_{1}^{\prime}$. Das harmonische Mass der Menge $I^{*} M, \omega\left(z, I^{*} M, R^{\prime}\right)$, ist dann und nur dann Null, wenn das harmonische Mass der Menge $M \cap M_{1}$ Null ist. Aus der Gleichheit

$$
E \omega\left(z, I^{*} M, R^{\prime}\right)=E I \omega(z, M, R)=\omega\left(z, M \cap M_{1}, R\right)
$$

sieht mann nähmlich, dass aus

und

$$
\omega\left(z, M \cap M_{1}, R\right)=0, \quad E \omega\left(z, I^{*} M, R^{\prime}\right)=0
$$

$$
\omega\left(z, I^{*} M, R^{\prime}\right)=\operatorname{IE\omega }\left(z, I^{*} M, R^{\prime}\right)=0
$$

folgt. Umgekehrt, aus $\omega\left(z, I^{*} M, R^{\prime}\right)=0$ folgt

$$
E \omega\left(z, I^{*} M, R^{\prime}\right)=0 \quad \text { und } \quad \omega\left(z, M \cap M_{1}, R\right)=0 .
$$

Es sei $M^{\prime}$ eine beliebige messbare Menge $M^{\prime} \cong M_{1}^{\prime}$. Dann ist $\omega\left(z, M^{\prime}, R^{\prime}\right)$ Null auf $\partial R^{\prime}$ und deshalb ist $\omega\left(z, M^{\prime}, R^{\prime}\right) \in \mathfrak{u}^{\prime}$. Wir bezeichnen mit $\mathfrak{R}$ bzw. $\mathfrak{M}_{\varepsilon}$ die Menge jener Punkte $e^{i 0} \in \widetilde{\mho}$ für die $E \omega\left(\varphi\left(e^{i 0}\right), M^{\prime}, R^{\prime}\right)=1$ bzw. $\varepsilon \leqq$ $E \omega\left(\varphi\left(e^{i 0}\right), M^{\prime}, R^{\prime}\right) \leqq 1-\varepsilon$ ist, und $M$ bzw. $M_{\varepsilon}$ sei die Menge der Punkte des idealen Randes der Fläche $R$ welche $\mathfrak{M}$ (bzw. $\mathfrak{M}_{\varepsilon}$ ) als Bild hat. Es ist

$$
\begin{aligned}
& \varepsilon \omega\left(z, M_{\varepsilon}, R\right) \leqq E \omega\left(z, M^{\prime}, R^{\prime}\right), \\
& E \omega\left(z, M^{\prime}, R^{\prime}\right)+\varepsilon \omega\left(z, M_{\varepsilon}, R\right) \leqq 1
\end{aligned}
$$

Daraus folgt

$$
\begin{aligned}
& \varepsilon I \omega\left(z, M_{\varepsilon}, R\right) \leqq I E \omega\left(z, M^{\prime}, R^{\prime}\right), \\
& I E \omega\left(z, M^{\prime}, R^{\prime}\right)+\varepsilon I \omega\left(z, M_{\varepsilon}, R\right) \leqq 1 .
\end{aligned}
$$

Wenn man beachtet, dass $\operatorname{IE} \omega\left(z, M^{\prime}, R^{\prime}\right)=\omega\left(z, M^{\prime}, R^{\prime}\right)$ ist, so folgt aus der ersten Ungleichung, dass $I \omega\left(\varsigma^{\prime}\left(t^{\prime}\right), M_{\varepsilon}, R\right)$ fast überall auf $C^{\prime} \mathfrak{l}^{\prime}$ einen nullen 
Winkelgrenzwert hat und aus der zweiten, dass $I \omega\left(\varphi^{\prime}\left(t^{\prime}\right), M_{\Sigma}, R\right)$ auch auf $\mathfrak{X}^{\prime}$ fast überall den Winkelgrenzwert Null hat. Es ist also $I \omega\left(z, M_{\S}, R\right)=0$. Da aber $M_{\Im} \subseteq M_{1}$ ist, so folgt

$$
\omega\left(z, M_{\varepsilon}, R\right)=\omega\left(z, M_{\varepsilon} \cap M_{1}, R\right)=E I \omega\left(z, M_{\varepsilon}, R\right)=0
$$

für jedes $\varepsilon$. Daraus kann man ersehen, dass $E \omega\left(\varphi(t), M^{\prime}, R^{\prime}\right)$ fast überall auf $\mathfrak{M}$ den Winkelgrenzwert 1 und fast überall auf $C \mathfrak{M}$ den Winkelgrenzwert 0 hat. Wir werden

$$
M=E^{*} M^{\prime}
$$

stellen und es ist $E \omega\left(z, M^{\prime}, R^{\prime}\right)=\omega\left(z, E^{*} M^{\prime}, R\right)$. Die Abbildung $E^{*}$ führt die messbaren Teilmengen der Menge $M_{1}^{\prime}$ in messbare Teilmengen der Menge $M_{1}$ über. Sie ist in einem gewissen Sinne die Umkehrung der Abbildung $I^{*}$, denn es ist

$$
\omega\left(z, I^{*} E^{*} M^{\prime}, R^{\prime}\right)=I \omega\left(z, E^{*} M^{\prime}, R\right)=I E \omega\left(z, M^{\prime}, R^{\prime}\right)=\omega\left(z, M^{\prime}, R\right),
$$

woraus

$$
I^{*} E^{*} M^{\prime}=M^{\prime}
$$

folgt, mit Ausnahme einer Menge vom Masse Null. Weiter ist

$$
\omega\left(z, E^{*} I^{*} M, R\right)=E \omega\left(z, I^{*} M, R^{\prime}\right)=E I \omega(z, M, R)=\omega\left(z, M \cap M_{1}, R\right)
$$

und also $E^{*} I^{*} M=M \cap M_{1}$ mit Ausnahme einer Menge vom Masse Null.

Für zwei punktfremde messbare Mengen $N_{1}, N_{2}$ des idealen Randes von $R$ ist

$$
\begin{gathered}
\omega\left(z, I^{*}\left(N_{1} \cup N_{2}\right), R^{\prime}\right)=I \omega\left(z, N_{1} \cup N_{2}, R\right)=I \omega\left(z, N_{1}, R\right) \\
+I \omega\left(z, N_{2}, R\right)=\omega\left(z, I^{*} N_{1}, R^{\prime}\right)+\omega\left(z, I^{*} N_{*}, R^{\prime}\right),
\end{gathered}
$$

woraus

$$
\begin{aligned}
& I^{*} N_{1} \cap I^{*} N_{2}=\phi, \\
& I^{*}\left(N_{1} \cup N_{2}\right)=I^{*} N_{1} \cup I^{*} N_{2} \cup \quad \text { Menge vom Masse Null }
\end{aligned}
$$

folgt. Ähnlicherweise ist für zwei punktfremde messbare Mengen $N_{1}^{\prime}, N_{2}^{\prime}$ des idealen Randes von $R^{\prime}$

$$
\begin{aligned}
& \omega\left(z, E^{*}\left(N_{1}^{\prime} \cup N_{2}^{\prime}\right), R\right)=E \omega\left(z, N_{1}^{\prime} \cup N_{2}^{\prime}, R^{\prime}\right)=E \omega\left(z, N_{1}^{\prime}, R^{\prime}\right) \\
& \quad+E \omega\left(z, N_{2}^{\prime}, R^{\prime}\right)=\omega\left(z, E^{*} N_{1}^{\prime}, R\right)+\omega\left(z, E^{*} N_{2}^{\prime}, R\right)
\end{aligned}
$$

woraus

$$
E^{*} N_{1}^{\prime} \cap E^{*} N_{2}^{\prime}=\phi
$$




$$
E^{*}\left(N_{1}^{\prime} \cup N_{2}^{\prime}\right)=E^{*} N_{1}^{\prime} \cup E^{*} N_{2}^{\prime} \cup \quad \text { Menge vom Masse Null }
$$

folgt.

Ist $M \subseteq M_{1}$ eine unteilbare Menge, so ist es auch $I^{*} M$. Erstens ist das harmonische Mass von $I^{*} M$ offenbar positiv. Zweitens ist

$$
\omega\left(z, I^{*} M, R^{\prime}\right)=I \omega(z, M, R)
$$

nach 10. eine minimale Funktion und da sie vom Null verschieden ist, so ist $I^{*} M$ nach Satz 3 eine unteilbare Menge. Umgekehrt, ist $M^{\prime} \cong M_{1}^{\prime}$ eine unteilbare Menge, so ist $E^{*} M^{\prime}$ auch unteilbar. Erstens ist das harmonische Mass von $E^{*} M^{\prime}$ offenbar positiv. Zweitens ist

$$
\omega\left(z, E^{*} M^{\prime}, R\right)=E \omega\left(z, M^{\prime}, R^{\prime}\right)
$$

nach 10. eine minimale Funktion und nach Satz 3 ist $E^{*} M^{\prime}$ eine unteilbare Menge.

Es sei $u^{\prime} \in \mathfrak{U}^{\prime}$ und $\mathfrak{M}^{\prime}(s)$ die Menge jener $e^{i \theta^{\prime}} \in \mathfrak{M}_{1}^{\prime}$, für welche $u^{\prime}\left(\varphi^{\prime}\left(e^{i \theta^{\prime}}\right)\right) \leqq s$ ist; $M^{\prime}(s)$ sei die Menge des idealen Randes von $R^{\prime}$, die $\mathfrak{M}^{\prime}(s)$ als Bild hat. Dann ist $\omega\left(z, \mathfrak{M}^{\prime}(s), R^{\prime}\right)$ eine in Bezug auf $s$ nichtabnähmende Funktion und

$$
u^{\prime}(z)=\int_{0}^{\infty} s d \omega\left(z, \mathfrak{M}^{\prime}(s), R^{\prime}\right)
$$

Daraus folgt

$$
E u^{\prime}(z)=\int_{0}^{\infty} s d \omega\left(z, E^{*} \mathfrak{M}^{\prime}(s), R\right)
$$

Ähnlicherweise, wenn wir für ein $u \in \mathfrak{U}$ mit $\mathfrak{M}(s)$ die Menge jener $e^{i \theta} \in \mathfrak{F}$, für welche $u\left(\varphi\left(e^{i \theta}\right)\right) \leqq s$ ist bezeichnen, und $M(s)$ die Menge des idealen Randes von $R$ ist, die $\mathfrak{R}(s)$ als Bild hat, so ist $\omega(z, M(s), R)$ eine in Bezug auf $s$ nichtabnähmende Funktion und

$$
u(z)=\int_{0}^{\infty} s d \omega(z, M(s), R) .
$$

Daraus folgt

$$
I u(z)=\int_{0}^{\infty} s d w\left(z, I^{*} M(s), R^{\prime}\right)
$$

13. Für einige Anwendungen, die wir im letzten Abschnitt geben wollen, ist es nützlich die geometrische Struktur der Menge $\mathfrak{M}_{1}$ näher zu untersuchen. Wir werden mit $\xi(z)$ die Funktion bezeichnen, welche auf $\Delta$ gleich 1 und auf $R-\Delta$ gleich 0 ist, und $\mathfrak{M}_{1}^{*}$ (bzw. $\mathfrak{M}_{0}^{*}$ ) sei die Menge der Punkte $e^{i \theta}$, für welche 
$\xi(\varphi(t))$ den Winkelgrenzwert 1 (bzw. 0) hat. $M_{1}^{*}$ (bzw. $M_{0}^{*}$ ) sei die Menge der Punkte des idealen Randes, die $\mathfrak{M}_{1}^{*}$ (bzw. $\mathfrak{M}_{0}^{*}$ ) als Bild hat. Wir wollen mittels der Konstruktion von Lusin und Priwaloff [9] zeigen, dass $M_{1}^{*} \cong M_{1}$ (bzw. $M_{0}^{*} \cong M_{0}$ ) ist, mit der Ausnahme einer Menge vom Masse Null.

Es ist zu zeigen dass $\mathfrak{M}_{1}^{*} \cap \mathfrak{M}_{0}$ (bzw. $\mathfrak{M}_{0}^{*} \cap \mathfrak{M}_{1}$ ) vom Masse Null ist. Wir nehmen an, dass diese Menge positives Mass hat. Es sei $\Delta\left(e^{i \theta}, r\right), r>\frac{\sqrt{2}}{2}$, die Menge

$$
r<|t|<1, \quad \mid \arg \frac{e^{i 0}-t}{e^{i 0}}<\frac{\pi}{4}
$$

und $\mathfrak{M}_{1 r}$ sei die Menge jener Punkte $e^{i 0} \in \mathfrak{R}_{1}^{*} \cap \mathfrak{M}_{0}$, für welche

$$
\Delta\left(e^{i \theta}, r\right) \subseteq \varphi^{-1}(\Delta)
$$

was gleichbedeutend mit $\xi(\varphi(t))=1$ für jedes $t \in \Delta\left(e^{i 0}, r\right)$ ist. $\mathrm{Da}$

$$
\mathfrak{M}_{1}^{*} \cap \mathfrak{M}_{0} \subseteq \underset{V}{\bigcup_{2 / 2<r<1}} \mathfrak{M}_{1 r}
$$

ist, kann man ein $r$ finden, $\frac{\sqrt{2}}{2}<r<1$ so dass $\mathfrak{M}_{1 r}$ positives Mass hat. Es sei $\mathfrak{R}$ eine perfekte Menge $\mathfrak{N} \subseteq \mathfrak{M}_{1 r}$, welche positives Mass hat und $G$ sei die offene Menge

$$
G=\bigcup_{e^{i \theta \in \mathbb{R}}} \Delta\left(e^{i \theta}, r\right)
$$

Sie besteht, wie leicht $z u$ ersehen ist, aus endlich vielen Jordanschen Gebieten mit rektifizierbarem Rande

$$
G=\bigcup_{j=1}^{k} G_{j}, \quad G_{j}=\bigcup_{e^{i \theta} \in \mathfrak{N}_{j}} \Delta\left(e^{i \theta}, r\right),
$$

wo

$$
\mathfrak{R}=\bigcup_{j=1}^{k} \mathfrak{N}_{j}
$$

ist. Daraus ist zu ersehen, dass ein $\Re_{j}$ positives Mass haben muss. Wir bilden die harmonische Funktion $\omega\left(t, \Re_{j}, G_{j}\right)$, welche nicht Null ist. Die Funktion $u^{* *}(t)$ gleich $\omega\left(t, \Re_{j}, G_{j}\right)$ in $G_{j}$ und gleich Null in $\varphi^{-1}(\Delta)-G_{j}$ ist subharmonisch in $\varphi^{-1}(\Delta)$ und dasselbe gilt für

$$
u^{*}(t)=\sup _{t \in\left(T^{\prime}\right)} u^{* *}(T(t))
$$

Die Funktion $u(z)=u^{*}\left(\varphi^{-1}(z)\right)$ ist, wie man leicht sieht, eindeutig und sub- 
harmonisch auf $\Delta$, Null auf $\partial \Delta$ und kleiner als 1 . Aus der Extremaleigenschaften der Funktion $I 1$ folgt

$$
u(z) \leqq I 1(z) \leqq E I 1(z)
$$

und

$$
\omega\left(t, \Re_{j}, G_{j}\right) \leqq u^{*}(t)=u(\varphi(t)) \leqq E I 1(\varphi(t)) .
$$

Da $\omega\left(t, \Re_{j}, G_{j}\right)$ fast überall auf $\mathfrak{N}_{j}$ den Winkelgrenzwert in Bezug auf $G_{j}, 1$ hat, so ist

$$
\lim _{r \rightarrow 1} \operatorname{EI}\left(\varphi\left(r e^{i \theta}\right)\right)=1
$$

für fast alle $e^{\imath \theta} \in \mathfrak{R}_{j}$, was der Beziehung $\mathfrak{N}_{j} \subseteq \mathfrak{M}_{0}$ widerspricht. Ähnlicherweise beweist man, dass $\mathfrak{M}_{0}^{*} \cap \mathfrak{M}_{1}$ das Mass Null hat.

\section{Andere Arten von Unteilbarkeiten}

14. Die unteilbaren Mengen haben uns erlaubt verschiedene Eigenschaften der Riemannschen Flächen aus der Klasse $O_{I I B}-O_{G} \mathrm{zu}$ erörtern und zu verallgemeinern. Der Parallelismus, der zwischen den Klassen $O_{H B}$ und $O_{H D}$ existiert, flösst uns die Idee ein, eine neue Unteilbarkeit einzuführen, welche uns erlaubt für die Klasse $H D$ dieselben Schritte wie für die Klasse $H B$ zu unternehmen.

Es sei $R \notin O_{G}$ eine Riemannsche Fläche und $\subseteq$ eine Menge von Funktionen auf $R$. Wir nehmen an, dass für jedes $u(z) \in \Subset, u(\varphi(t))$ fast überall auf $|t|=1$ Winkelgrenzwerte besitzt.

Definition 3. Eine Menge von Punkten des idealen Randes $M$ heisst $\mathfrak{\subseteq}$ unteilbar, wenn sie positives Mass hat und wenn für jedes $u(z) \in \Xi, u(\varphi(t))$ fast überall auf dem Bild $\mathfrak{M}$ von $M$ denselben Winkelgrenzwert hat.

Ist eine Menge $M$ unteilbar, so ist sie für jedes $\Subset, \subseteq$-unteilbar. Aus dem

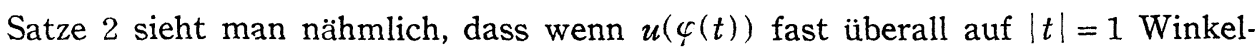
grenzwerte hat, so sind diese Winkelgrenzwerte fast überall auf $\mathfrak{M}$ konstant. Wir werden uns beschränken für $\varsigma$ die Mengen $H B, H D$ und $H B D$ zu betrachten. Wir werden erst beweisen, dass Unteilbarkeit und $H B$-Unteilbarkeit zusammenfallen. Ist eine Menge $M$ nicht unteilbar, so kann man zwei messbare und vom positiven Masse Mengen $\mathrm{M}_{1}, \mathrm{M}_{2}$ finden, so dass $M=M_{1} \cup M_{2}, M_{1} \cap M_{2}$ $=\phi$ ist. Das harmonische Mass $\omega\left(z, M_{1}, R\right)$ gehört der Menge $H B$ an und $\omega\left(c(t), M_{1}, R\right)$ hat fast überall auf $\mathfrak{M}_{1}$ den Winkelgrenzwert 1 und fast überall auf $\mathfrak{M}_{2}$ den Winkelgrenzwert $0 . \quad M$ ist somit nicht $H B$-unteilbar. Ist $M$ 
unteilbar, so ist sie, wie man gesehen hat, auch $H B$-unteilbar. Eine unteilbare Menge ist eine HB-unteilbare Menge und umgekehrt.

Jede $H B$ (bzw. HD)-unteilbare Menge liegt auf einer bestimmten idealen Randkomponente mit Ausnahme einer Menge von harmonischem Mass Null. Der Beweis von 5. gilt nähmlich auch für $H D$-unteilbare Mengen, denn die harmonischen Masse der Portionen haben endliches Dirichlet-Integral.

Definition 4. Eine Riemannsche Fläche gehört der Klasse $U_{\Im}$ an, u'enn ihr idealer Rand eine $\subseteq$-unteilbare Menge enthält.

Es ist offenbar

$$
U=U_{H B} \subseteq U \Subset
$$

für jedes $\Subset$. Ähnlich wie im 7 . beweist man folgenden

SATZ 7. Es ist

$$
\begin{aligned}
& O_{H B}-O_{G} \subset U_{H B} \subset O_{A B}-O_{G} \\
& O_{H D}-O_{G} \subset U_{H D} \subset O_{A D}-O_{r}
\end{aligned}
$$

15. T. Kuroda ([7] sieke auch [1]), hat folgende Definition eingeführt: Das Gebiet $R^{\prime}$ mit analytischem Rande gehört der Klasse $S_{X},(X=H, A$; $Y=B, D, B D)$ an, wenn jede Funktion $u(z) \in X Y$, die auf $\partial R^{\prime}$ Null ist, auf $R^{\prime}$ identisch verschwindet. Durch $u(z) \in A$ verstehen wir, dass $u(z)=\operatorname{Re} w(z)$, wo $w(z)$ eine eindeutige analytische Funktion ist.

Notwendig und hinreichend damit $R^{\prime} \in S O_{H B}$ ist, ist, dass

$$
I 1(z) \equiv \omega\left(z, M_{1}^{\prime}, R^{\prime}\right) \equiv 0,
$$

wo $I$ in Bezug auf das Paar $\left(R^{\prime}, R\right)$ definiert ist. Denn ist $I 1(z) \neq 0$, so hat man schon eine beschränkte harmonische Funktion auf $R^{\prime}$, die auf $\partial R^{\prime}$ verschwindet und $R^{\prime} \notin S O_{H B}$. Aus der Extremaleigenschaft der Funktion $I 1(z)$ folgt $|u(z)| \leqq c I 1(z)$, wo $c=\sup |u(z)|$ ist, für jede beschränkte harmonische Funktion auf $R^{\prime}$, die auf $\partial R^{\prime}$ verschwindet. Aus $I 1(z) \equiv 0$ folgt dann $u(z) \equiv 0$ und $R^{\prime} \in S O_{I I B}$.

Weiter bezeichnet T. Kuroda [8] mit $O_{X Y}^{0}$ die Klasse jener Riemannschen Flächen $R$ für welche jedes Gebiet $R^{\prime}$ mit analytischen Rande der Klasse $S O_{X}$ angehört. T. Kuroda hat bewiesen, dass die Riemannschen Flächen aus der Klasse $O_{A B}^{0}$ eine wichtige Eigenschaft besitzen: ist $w^{(}(z)$ eine eindeutige meromorphe Funktion auf $R \in O_{A B}^{0}$, so hat die inverse Funktion $z=z(w)$ von $w=w(z)$ 
die Eigenschaft von Iversen [21].

Hilfssatz 4. [1], [12]. Es ist

$$
S O_{H D}=S O_{I H B n} \supseteqq S O_{H B}
$$

Die Beweismethode ahmt die Methode nach, die für die Beziehung $O_{H B}-O_{G}$ $\leqq O_{\mu n}$ benutzt wird ([16], Seite $\left.365-367,[24]\right)$. Es sei $R$ eine beliebige Riemannsche Fläche, $R^{\prime}$ ein Gebiet auf $R$ mit analytischem Rande, $\left\{R_{n}\right\}$ eine Ausschöpfung von $R$ und $R_{n}^{\prime}$ die Komponente von $R_{n} \cap R^{\prime}$, die einen bestimmten Punkt $z_{0}$ enthält. Mit $\gamma_{n}\left(z, z_{0}\right)$ werden wir die in $R_{n}^{\prime}-\left\{z_{0}\right\}$ harmonische Funktion bezeichnen, welche in $z_{0}$ die Entwicklung

$$
r\left(z, z_{0}\right)=\log \frac{1}{\left|z-z_{0}\right|}+\text { harmonische Funktion }
$$

hat und so, dass

$$
\frac{\partial \gamma_{n}}{\partial_{n}}=0 \text { auf } \partial R_{n}^{\prime} \cap R^{\prime}, \cdot \quad r_{n}=0 \text { auf } \partial R_{n}^{\prime} \cap R_{n}
$$

ist. Es sei

$$
2 \pi v_{n}\left(z, z_{0}\right)=\gamma_{n}\left(z, z_{\jmath}\right)-g_{n}\left(z, z_{0}\right),
$$

wo $g_{n}\left(z, z_{n}\right)$ die Greensche Funktion des Gebietes $R_{n}^{\prime}$ ist. Die Funktion $v_{n}\left(z, z_{0}\right)$ ist auf $R_{n}^{\prime}$ harmonisch und verschwindet auf $\partial R_{n}^{\prime} \cap R_{n}$. Es sei $u(z)$ eine harmonische Funktion auf $\bar{R}_{n}^{\prime}$, welche auf $\partial R_{n}^{\prime} \cap R_{n}$ verschwindet. Aus der Greenschen Transformationsformell (man kann immer die Ausschöpfung $\left\{R_{n}\right\}$ so nehmen, dass $v_{n}\left(z, z_{0}\right)$ auf $\bar{R}_{n}^{\prime}$ harmonisch ist) ergibt sich

$$
\begin{aligned}
& D_{R_{n^{\prime}}}\left(u, v_{n}\right) \equiv \iint_{R_{u^{\prime}}}\left(\frac{\partial u}{\partial x} \frac{\partial v_{n}}{\partial x}+\frac{\partial u}{\partial y} \frac{\partial v_{n}}{\partial y}\right) d x d y \\
& \quad=\int_{\partial R_{n^{\prime} \cap R^{\prime}}} u \frac{\partial v_{n}}{\partial n} d s:=-\frac{1}{2 \pi} \int_{\partial R^{\prime} n \cap R^{\prime}} u(\zeta) \frac{\partial g_{n}\left(\zeta, z_{0}\right)}{\partial n}|d \zeta|=u\left(z_{0}\right) .
\end{aligned}
$$

Insbesondere ist für $u=v_{n}$

$$
D_{R_{n^{\prime}}}\left(v_{n}\right) \equiv \iint_{R_{n^{\prime}}}\left(\left(\frac{\partial v_{n}}{\partial x}\right)^{2}+\left(\frac{\partial v_{n}}{\partial y}\right)^{2}\right) d x d y=v_{n}\left(z_{0}, z_{0}\right)>0
$$

und für $u=v_{m}, m>n$,

$$
D_{R_{n^{\prime}}}\left(v_{n}, v_{m}\right)=v_{m}\left(z_{0}, z_{0}\right)
$$

Daraus folgt 


$$
\begin{aligned}
0 & <D_{R_{n^{\prime}}}\left(v_{m}-v_{n}\right)=D_{R_{n^{\prime}}}\left(v_{m}\right)+D_{R_{n^{\prime}}}\left(v_{n}\right)-2 D_{R_{n^{\prime}}}\left(v_{n}, v_{m}\right) \\
& \leqq v_{m}\left(z_{0}, z_{0}\right)+v_{n}\left(z_{0}, z_{0}\right)-2 v_{m}\left(z_{0}, z_{0}\right)=v_{n}\left(z_{0}, z_{0}\right)-v_{m}\left(z_{0}, z_{0}\right) .
\end{aligned}
$$

Die Zahlenfolge $\left\{v_{n}\left(z_{0}, z_{0}\right)\right\}$ nimmt also mit wachsendem $n$ monoton ab; es existiert daher der Grenzwert

$$
\lim _{n \rightarrow \infty} v_{n}\left(z_{0}, z_{0}\right) \geq 0
$$

Weiter folgt, dass die Folge $v_{n}\left(z, z_{0}\right)$ in Norm konvergiert, und deshalb konvergiert sie auch im gewöhnlichem Sinne ([16], Seite 291). Die Grenzfunktion

$$
v\left(z, z_{0}\right)=\lim _{n \rightarrow \infty} v_{n}\left(z, z_{0}\right)
$$

ist harmonisch in $R^{\prime}$ und verschwindet auf $\partial R^{\prime}$. Forner folgt, dass

$$
\lim _{n \rightarrow \infty} \gamma_{n}\left(z, z_{0}\right)=\gamma\left(z, z_{0}\right)=2 \pi v\left(z, z_{0}\right)+g\left(z, z_{0}\right)
$$

existiert, wo $g\left(z, z_{0}\right)$ die Greensche Funktion von $R^{\prime}$ ist. Es sei $G$ ein Jordansches Gebiet, das den Punkt $z_{0}$ enthält, $\bar{G} \subset R^{\prime}$. In $R^{\prime}-\bar{G}$ ist $g\left(z, z_{0}\right)$ beschränkt ; auch $\gamma\left(z, z_{0}\right)$ ist hier beschränkt, denn es ist

$$
\sup _{z \in R_{n^{\prime}}-\sigma_{i}} \gamma_{n}\left(z, z_{0}\right)=\sup _{z \in A_{i}} \gamma_{n}\left(z, z_{0}\right)
$$

und daher ist

$$
\sup _{z \in R^{\prime}-G_{i}} \gamma\left(z, z_{0}\right)=\sup _{z \in \partial G} \gamma\left(z, z_{\cap}\right)
$$

Daraus folgt $v(z) \in H B$. Da $v_{n}(z) \in H D$ und gegen $v(z)$ in Norm konvergieren, so ist $v(z) \in H D$. Es sei $u(z)$ harmonisch in $R^{\prime}$ und Null auf $\partial R^{\prime}$. Es ist offenbar

$$
D_{R^{\prime}}(u, v)=u\left(z_{0}\right) .
$$

Gehört $R^{\prime}$ der Klasse $S O_{H D}$ nicht an, so existiert ein $u(z)$ mit $u\left(z_{0}\right) \neq 0$. Daraus folgt $v(z) \neq 0$ und das Gebiet $R^{\prime}$ gehört somit der Klasse $S O_{m \text { nn }}$ nicht an. Es ist also

$$
S O_{H D}=S O_{I H B N} \supseteqq S O_{H I}
$$

was $\mathrm{zu}$ beweisen war.

HilfsSATz 5. Es sei u(z) eine harmonische Funktion mit endlichem DirichletIntegral auf einer Riemannschen Fläche R. Wenn fast ïberall auf $|t|=1$ die Winkelgrenzwerte von $u(\varphi(t))$ kleiner als $N$ sind, so ist $u(z)<N$. 
Es sei $\Delta$ die offene Menge, wo $u(z)>N$ ist und $\xi(z)$ die Funktion gleich 1 auf $\Delta$ und gleich 0 auf $R-\Delta$. Überall, wo $u(c(t))$ den Winkelgrenzwert kleiner als $N$ hat, hat $\xi(\varphi(t))$ den Winkelgrenzwert Null. Mit den Bezeichnungen, die wir in 13. eingeführt haben, ist das Lebesguesche Mass von $\mathfrak{M}_{0}^{*}$ gleich $2 \pi$. Aus

$$
\mathfrak{M}_{0}^{*} \subseteq \mathfrak{M}_{0}
$$

folgt, dass auch $\mathfrak{M}_{0}$ das Lebesguesche Mass $2 \pi$ hat und $\mathfrak{M}_{1}$ ist somit eine Menge vom Masse Null. Daraus folgt $E I 1 \equiv 0$ und $I 1 \equiv 0$. Das ist aber eine hinreichende Bedingung, dass jede Komponente $R^{\prime}$ aus $\Delta$ der Klasse $S O_{H B}$ angehört. Aus

$$
S O_{H B} \subseteq S O_{H D}
$$

folgt, dass $R^{\prime} \in S O_{H D}$ im Widerspruch mit der Existenz der Funktion $u(z)-N$, Null auf dem Rande von $R^{\prime}$ und mit beschränktem Dirichlet-Integral. Die Menge $\Delta$ ist somit leer und $u(z)<N$, was $z$ u beweisen war.

Hat $u(\varphi(t))$ fast überall den Winkëlgrenzwert Null, so folgt sofort $u(z) \equiv 0$.

16. SATz 8. Jede harmonische Funktion mit endlichem Dirichlet-Integral ist als Differenz von zwei positiven harmonischen Funktionen mit endlichem Dirichlet-Integral darstellbar.

Es sei $\left\{R_{n}\right\}$ eine Ausschöpfung der Fläche $R$ und $\underline{\omega}_{n}(z)$ die auf $\overline{R_{n}}-R_{0}$ harmonische Funktion welche auf $\partial R_{0}$ gleich 0 und auf $\partial R_{n}$ gleich 1 ist. Es sei $u(z)$ eine harmonische Funktion mit endlichem Dirichlet-Integral und $R^{+}$die offene Menge, wo $u(z)>0$ ist. Wir nehmen an, dass $R_{0} \cap R^{+}=\phi$ ist und bezeichnen mit $u_{n}(z)$ die auf $R_{n}$ harmonische Funktion, welche auf $\bar{R}_{n}$ stetig ist und für welche

$$
u_{n}(z)=\left\{\begin{array}{cll}
u(z) & \text { für } & z \in \partial R_{n} \cap R^{+} \\
0 & \text { für } & z \in \partial R_{n} \cap\left(R-R^{+}\right)
\end{array}\right.
$$

ist. Auf $R_{n}$ ist $u(z) \leqq u_{n}(z)$ und daher ist $u_{n}(z) \leqq u_{n+1}(z)$. Nach dem Harnackschen Prinzip konvergiert die Folge $u_{n}(z)$ entweder gegen eine harmonische Funktion $u^{+}(z)$ oder gegen unendlich. Wir wollen zeigen, dass hier der erste Fall zutritt.

Aus der Greenschen Transformationsformel folgt

$$
\int_{\partial R_{0}} u_{n} \frac{\partial \underline{\omega}_{n}}{\partial n} d s=\int_{\partial R_{n}} u_{n} \frac{\partial \underline{\omega}_{n}}{\partial n} d s
$$


Weiter ist

$$
\int_{\partial R_{n}} u_{n} \frac{\partial \underline{\omega}_{n}}{\partial n} d s=\int_{R^{+} \cap \partial R_{n}} u \frac{\partial \underline{\omega}_{n}}{\partial n} d s=D_{R_{u} \subset R^{+}}\left(u, \underline{\omega}_{n}\right)
$$

Nach der Schwarzschen Ungleichung ist

$$
\left|D_{R_{n} \wedge R^{-}}\left(u, \underline{\omega}_{n}\right)\right|^{2} \leqq D_{R_{n} \cap R^{+}}(u) \cdot D_{R_{n} \cap R^{+}}\left(\underline{\omega}_{n}\right) \leqq D_{l_{i}}(u) \cdot D_{R_{u}-\bar{H}_{u}}\left(\underline{\omega}_{n}\right) .
$$

Daraus folgt

$$
\int_{\partial R_{0}} u_{n} \frac{\partial \underline{\underline{\omega}}{ }_{n}}{\partial n} d s \leqq \sqrt{D_{R}(u) \cdot D_{R_{n}-\bar{R}_{0}}\left(\underline{\omega}_{n}\right)}
$$

und

$$
\inf _{z \in \partial R_{0}} u_{n}(z) \cdot D_{R_{n}-\bar{R}_{0}}\left(\underline{\omega}_{n}\right)=\inf _{z \in \partial R_{0}} u_{n}(z) \int \frac{\partial \underline{\omega}_{n}}{\partial n} d s \leqq \vee D_{R}(u) \cdot \bar{D}_{R_{n}-\bar{R}_{0}}\left(\omega_{n}\right)
$$

oder

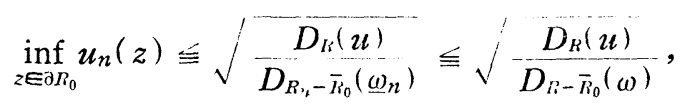

wo

$$
\underline{\omega}(z)=\lim _{n \rightarrow \infty} \underline{\omega}_{n}(z) \neq 1
$$

ist. Daraus folgt, dass $u_{n}(z)$ nicht gegen unendlich konvergieren kann und es ist

$$
\lim _{n \rightarrow \infty} u_{n}(z)=u^{+}(z)
$$

Die Funktion $u^{+}(z)$ hat endliches Dirichlet-Integral. Es ist

$$
D_{R}\left(u^{+}\right)=\lim _{m \rightarrow \infty} D_{R_{m}}\left(u^{+}\right)=\lim _{m \rightarrow \infty} \lim _{u \rightarrow \infty} D_{R_{m}}\left(u_{n}\right) \leqq \lim _{n \rightarrow \infty} D_{R_{n}}\left(u_{n}\right) .
$$

Nach dem Dirichletschen Prinzip ist

$$
D_{R_{n}}\left(u_{n}\right) \leqq D_{R_{n} \cap R^{+}}(u) \leqq D_{R}(u),
$$

woraus $D_{R}\left(u^{+}\right) \leqq D_{R}(u)$ folgt. Die Funktion $u_{n}(z)-u(z)$ ist nichtnegativ und konvergiert gegen eine nichtnegative Funktion

$$
u^{-}(z)=\lim _{n \rightarrow \infty}\left(u_{n}(z)-u(z)\right)=u^{+}(z)-u(z)
$$

welche auch endliches Dirichlet-Integral hat. Es ist also $u(z)=u^{+}(z)-u^{-}(z)$, was $\mathrm{zu}$ beweisen war.

Bemerkung. Es sei $u(z)$ eine harmonische Funktion auf $R$ mit endlichem Dirichlet-Integral und $u\left(c ̧\left(e^{i \theta}\right)\right)$ der Winkelgrenzwert von $u(c ̧(t))$ im Punkte 
$e^{i 0}$, falls dieser existiert (er existiert für fast alle $\left.e^{i n}\right)$. Dann ist $u\left(\varphi\left(e^{i 0}\right)\right.$ ) eine summierbare Funktion.

$u(\varphi(t))$ ist als Differenz von zwei positiven harmonischen Funktionen durch das Poisson-Stieltjessche Integral darstellbar:

$$
u(\varphi(t))=\int_{0}^{2 \pi} P(\theta, t) d \mu(\theta)
$$

Es ist fast überall $u\left(\varphi\left(e^{i 0}\right)\right)=\mu^{\prime}(\theta)$, woraus die Behauptung folgt.

17. SATz 9. [17]. Es sei u(z) eine harmonische Funktion mit endlichem Dirichlet-Integral. $u(\varphi(t))$ ist durch ein Poisson-Lebesguesche Integral darstellbar:

$$
u(\varphi(t))=\int_{0}^{2 \pi} u\left(\varphi\left(e^{i)}\right)\right) P(\theta, t) d \theta
$$

Weiter ist $u(z)$ Grenzfunktion einer Folge von harmonischen, beschränkten Funktionen mit beschränktem Dirichlet-Integral.

Nach dem Satz 8 genügt es den Satz für positive Funktionen zu beweisen. Wir bezeichnen :

$$
\begin{aligned}
& u_{N}^{*}\left(e^{i \theta}\right)=\left\{\begin{array}{ccc}
u\left(\varphi\left(e^{i \theta}\right)\right) & \text { für } & u\left(\varphi\left(e^{i 0}\right)\right) \leqq N \\
N & \text { für } & u\left(\varphi\left(e^{i \theta}\right)\right) \leqq N,
\end{array}\right. \\
& u_{N}^{*}(t)=\int_{0}^{2 \pi} u_{N}^{*}\left(e^{i 0}\right) P(\theta, t) d \theta, \quad u_{N}(z)=u_{N}^{*}\left(\varphi^{-1}(z)\right) \text {. }
\end{aligned}
$$

Aus $u_{*}^{*}(t) \leqq u(\varphi(t))$ und $u_{N}^{*}(t) \leqq N$ folgt $u_{N}(z) \leqq u(z), u_{N}(z) \leqq N$. Wir werden mit $\Delta_{x}$ die offene Menge, wo $u(z)<N$ ist, bezeichnen und mit $u_{v n}(z)$ die in $R_{n}$ beschränkte und harmonische Funktion, die auf $\bar{R}_{n}$ stetig ist und für die

$$
u_{s n}(z)=\left\{\begin{array}{cll}
u(z) & \text { für } & z \in \partial R_{n} \cap \Delta_{n} \\
N & \text { für } & z \in \partial R_{n} \cap\left(R-\Delta_{N}\right)
\end{array}\right.
$$

ist. In $R_{n}$ ist offenbar

$$
u_{v}(z) \leqq u_{\text {vnn }}(z) \leqq u(z), \quad u_{\text {s } n}(z) \leqq N
$$

und daraus folgt in $R_{n}$

$$
u_{v, n+1}(z) \leqq u_{s, n}(z) \text {. }
$$

Die Folge $\left\{u_{s i n}(z)\right\}$ konvergiert nach dem Harnackschen Prinzip gegen eine harmonische Funktion $\bar{u}_{x}(z)$, für die $u_{v}(z) \leqq \bar{u}_{N}(z) \leqq u(z), \quad \bar{u}_{\mathrm{N}}(z) \leqq N$ gilt. 
Wenn $\xi_{x n}(z)$ in $R_{n} \cap \Delta_{v}$ gleich $u(z)$ und in $R_{n} \cap\left(R-\Delta_{*}\right)$ gleich $N$ ist, so folgt aus dem. Dirichletschen Prinzip

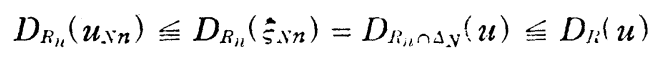

und weiter ist

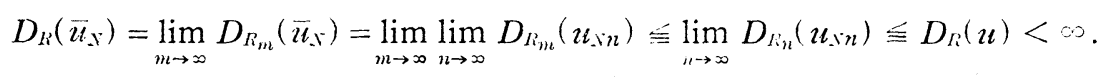

$\bar{u}_{x}(\varphi(t))$ hat somit fast überall auf $|t|=1$ Winkelgrenzwerte und aus

$$
\begin{aligned}
& \left.u_{N}^{+}(t)=u_{x}(c(t)) \leqq \bar{u}_{N}(c)(t)\right) \leqq u(c(t)), \\
& \bar{u}_{x}(\varphi(t)) \leqq N
\end{aligned}
$$

folgt dass $\bar{u}_{N}(\varphi(t))$ und $u_{N}(\varphi(t))$ fast überall dieselben Winkelgrenzwerten haben. Es ist also $\bar{u}_{X}(\varphi(t))=u_{S}(\varphi(t)), \bar{u}_{X}(z)=u_{X}(z)$ und

$$
D_{K_{i}}\left(u_{-}\right) \leqq D_{R}(u)<\infty .
$$

Wir stellen

$$
u_{\infty}^{*}(t)=\int_{0}^{2 \pi} u\left(c\left(e^{i \theta}\right)\right) P(\theta, t) d \theta, \quad u_{z}(z)=u_{\infty}^{*}\left(c_{1}^{-1}(z)\right)
$$

Es ist offenbar

$$
u_{i \infty}^{*}(t)=\lim _{i \rightarrow \infty} u_{i}^{*}(t), \quad u_{\infty}(z)=\lim _{i \rightarrow \infty} u_{N}(z) .
$$

Daher folgert man

$$
D_{R}\left(u_{\infty}\right)=\lim _{n \rightarrow \infty} D_{R_{u}}\left(u_{\infty}\right)=\lim _{n \rightarrow \infty} \lim _{X \rightarrow \infty} D_{R_{u}}\left(u_{n}\right) \leqq \lim _{Y \rightarrow \infty} D_{f_{i}}\left(u_{\Lambda}\right) \leqq D_{R_{i}}(u)<\infty .
$$

Die Funktion $u_{0}(z)=u(z)-u_{\infty}(z)$ ist harmonisch mit beschränktem DirichletIntegral und $u_{0}(c(t))$ hat fast überall auf $\mid t !=1$ den Winkelgrenzwert Null. Aus dem Hilfssatz 5 folgt dann $u_{0}(z) \equiv 0$ und $u(z)=u_{a}(z)$, was alle Behauptungen des Satzes bestätigt.

Aus diesem Satz folgt unmittelbar der

Folgesatz 1. Jede HD-unteilbare Menge ist eine HBD-unteilbare Menge und umgekehrt.

18. Wir haben eine eineindeutige Beziehung zwischen den $H B$-unteilbaren Mengen und den minimalen Funktionen in der Klasse $H B$ festgestellt. Eine ähnliche Beziehung besteht aber nicht mehr, wenn man $H B$ mit $H D$ ersetzt. 
Um dennoch eine ähnliche Beziehung festzustellen, müssen wir uns erstens nur auf die maximalen $H D$-unteilbaren Mengen beschränken. Das sind solche $H D$. unteilbaren Mengen $M$, für die man keine $H D$-unteilbare Menge $\bar{M}$ finden kann, so dass $\bar{M} \supseteqq M$ und

$$
\omega(2, \bar{M}-M, R)>0
$$

ist. Zweitens werden wir die Klasse $H D$ ein wenig erweitern müssen. Anstatt der positiven Funktionen aus der Klasse $H D$, betrachten wir die positiven Funktionen aus der Klasse $H D$. Diese Klasse wird folgenderweise definiert: $u(z) \in H D$, wenn

$$
u(z)=\lim _{n \rightarrow \infty} u_{n}(z), \quad u_{n}(z) \geq u_{n+1}(z) \geq 0, \quad u_{n}(z) \in H D
$$

ist. Wir bezeichnen

$$
\begin{gathered}
u^{*}\left(e^{i \theta}\right)=\lim _{u \rightarrow \infty} u_{n}\left(\varphi\left(e^{i 0}\right)\right), \\
u^{*}(t)=\int_{0}^{2 \pi} u^{*}\left(e^{i 0}\right) P(\theta, t) d \theta=\lim _{n \rightarrow \infty} \int_{0}^{2 \pi} u_{n}\left(\varphi\left(e^{i \theta}\right)\right) P(\theta, t) d \theta=u(\varphi(t))
\end{gathered}
$$

Daraus folgt, dass $u(\varphi(t))$ durch das Poisson-Lebesguesche Integral darstellbar ist und, dass

$$
u\left(\varphi\left(e^{i \theta}\right)\right)=u^{*}\left(e^{i \theta}\right)=\lim _{n \rightarrow \infty} u_{n}\left(\varphi\left(e^{i \theta}\right)\right)
$$

für fast alle $e^{i \theta}$ gilt.

Es seien $u_{\nu}(z) \quad(\nu=1,2, \ldots, k)$ harmonische Funktionen auf $R$, so dass $u_{*}(\varphi(t))$ fast überall auf $|t|=1$ Winkelgrenzwerte besitzt und

$$
u_{2}(\varphi(t))=\int_{0}^{2 \pi} u_{2}\left(\varphi\left(e^{i \theta}\right)\right) P(\theta, t) d \theta
$$

ist. Es sei

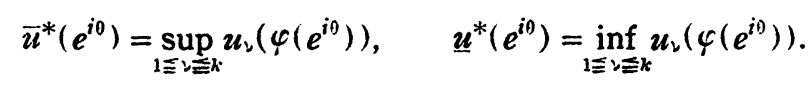

Beide sind summierbare Funktionen und automorph in Bezug auf $(T)$. Wir stellen

$$
\begin{aligned}
& \bar{u}^{*}(t)=\int_{0}^{2 \pi} \bar{u}^{*}\left(e^{i n}\right) P(\theta, t) d \theta, \\
& \underline{u}^{*}(t)=\int_{0}^{2 \pi} \underline{u}^{*}\left(e^{i n}\right) P(\theta, t) d \theta ; \\
& \bar{u}(z)=\bar{u}^{*}\left(\varphi^{-1}(z)\right), \quad \underline{u}(z)=\underline{u}^{*}\left(\varphi^{-1}(z)\right) .
\end{aligned}
$$


Wir nennen $\bar{u}(z)$ bzw. $\underline{u}(z)$ die obere bzw. untere Hülle von $\left\{u_{,}\right\}$und werden bezeichnen

$$
\bar{u}=\bigcup_{\nu=1}^{k} u_{\nu}, \quad \underline{u}=\bigcap_{\nu=1}^{k} u_{\nu} .
$$

Es ist $\underline{u}(z) \leqq u_{\nu}(z) \leqq \bar{u}(z) \quad(\nu=1,2, \ldots, k)$. Ist $u(z)$ so, dass $u(c,(t))$ durch das Poisson-Lebesguesche Integral darstellbar ist und $u(z) \geqq u_{i}(z)$ bzw. $u(z)$ $\leqq u_{\nu}(z),(\nu=1,2, \ldots, k)$, so ist

$$
u(z) \geqq \bar{u}(z) \text { bzw. } u(z) \leqq \underline{u}(z) .
$$

HilfsSATZ 6. Ist $u_{1}, u_{2}, \ldots, u_{k} \in H D(b z w . ~ H D)$ so ist auch

$$
\begin{array}{ll}
\bar{u}=\bigcup_{\nu=1}^{k} u_{\nu} \in H D & \text { (bzw. } H D) \\
\underline{u}=\bigcap_{\nu=1}^{k} u_{\nu} \in H D & \text { (bzw. HD). }
\end{array}
$$

Wir nehmen zuerst an, dass $u, \in H D(\nu=1,2, \ldots, k)$ und bezeichnen mit $\bar{u}_{n}(z)$ bzw. $\underline{u}_{n}(z)$ die in $R_{n}$ harmonische auf $\bar{R}_{n}$ stetige Funktion, für welche

$$
\begin{aligned}
& \bar{u}_{n}(z)=\sup _{1 \leqq \nu: k} u_{i}(z) \text { für } z \in \partial R_{n} \\
& \text { bzw. } \underline{u}_{n}(z)=\inf _{1 \leqq \unrhd \equiv k} u_{\downarrow}(z) \text { für } z \in \partial R_{n}
\end{aligned}
$$

ist. In $R_{n}$ ist $\underline{u}(z) \leqq \underline{u}_{n}(z) \leqq u_{\nu}(z) \leqq \bar{u}_{n}(z) \leqq \bar{u}(z) \quad(\nu=1,2, \ldots, k)$. Daraus folgt $\bar{u}_{n+1}(z) \geqq \bar{u}_{n}(z), \underline{u}_{n+1}(z) \leqq \underline{u}_{n}(z)$ und die Folgen $\left\{\bar{u}_{n}(z)\right\},\left\{\underline{u}_{n}(z)\right\}$ sind konvergent:

$$
\bar{u}_{\infty}(z)=\lim _{n \rightarrow \infty} \bar{u}_{n}(z), \quad \underline{u}_{\infty}(z)=\lim \underline{u}_{n}(z)
$$

Es sei

$$
\bar{\xi}(z)=\sup _{1 \leqq \nu \equiv k} u \cdot(z), \quad \underline{\xi}(z)=\inf _{1 \triangleq \nu \leqq k} u_{\nu}(z) \quad \text { für } \quad z \in R .
$$

Nach dem Dirichletschen Prinzip ist

$$
\begin{aligned}
& D_{l_{i_{n}}}\left(\bar{u}_{n}\right) \leqq D_{R_{n}}(\bar{\xi}) \leqq D_{R}(\bar{\xi}) \leqq \sum_{\nu=1}^{k} D_{R}\left(u_{\nu}\right), \\
& D_{R_{n}}\left(\underline{u}_{n}\right) \leqq D_{R_{n}}(\underline{\xi}) \leqq D_{R}(\underline{\xi}) \leqq \sum_{\nu=1}^{k} D_{R}\left(u_{\nu}\right) .
\end{aligned}
$$

Weiter ist 


$$
\begin{aligned}
& D_{R}\left(\bar{u}_{\infty}\right) \leqq \lim _{u \rightarrow \infty} D_{R_{n}}\left(\bar{u}_{n}\right) \leqq \sum_{i=1}^{k} D_{R}\left(u_{\nu}\right)<\infty, \\
& D_{R}\left(\underline{u}_{\infty}\right) \leqq \lim _{n \rightarrow \infty} D_{R_{n}}\left(\underline{u}_{n}\right) \leqq \sum_{\nu=1}^{k} D_{R}\left(u_{v}\right)<\infty .
\end{aligned}
$$

Die Funktion $\bar{u}_{\infty}(\varphi(t))$ bzw. $\underline{u}_{\infty}(\varphi(t))$ hat also fast überall auf $|t|=1$ Winkelgrenzwerte und aus

$$
\underline{u}(\varphi(t)) \leqq \underline{u}_{\infty}(\varphi(t)) \leqq u_{\nu}(\varphi(t)) \leqq \bar{u}_{\infty}(\varphi(t)) \leqq \bar{u}(\varphi(t)) \quad(\nu=1,2, \ldots, k)
$$

folgt

$$
\bar{u}\left(\varphi\left(e^{i 0}\right)\right)=\bar{u}_{\infty}\left(\varphi\left(e^{i 0}\right)\right), \quad \underline{u}\left(\varphi\left(e^{i 0}\right)\right)=\underline{u}_{\infty}\left(\varphi\left(e^{i 0}\right)\right)
$$

für fast alle $e^{i \theta}$. Da alle diese Funktionen durch das Poisson Lebesguesche Integral darstellbar sind, so ist

$$
\bar{u}(z)=\bar{u}_{\infty}(z) \in H D, \quad \underline{u}(z)=\underline{u}_{\infty}(z) \in H D
$$

Wir nehmen jetzt an, dass $u_{\nu}(z) \in \underline{H D}$ und es sei

$$
u_{\nu}(z)=\lim _{\mu \rightarrow \infty} v_{\nu \mu}(z), \quad v_{\nu \mu}(z) \geq v_{\nu, \mu+1}(z) \geqslant 0, \quad v_{\nu \mu}(z) \in H D .
$$

Wir stellen

$$
\bar{v}_{\mu}=\bigcup_{\nu=1}^{k} v_{\nu \mu} \in H D, \quad \underline{v}_{\mu}=\bigcap_{\nu=1}^{k} v_{\nu \mu} \in H D
$$

Es ist offenbar $\bar{v}_{\mu} \geqslant \bar{v}_{\mu+1} \geqslant 0, \underline{v}_{\mu} \geqslant \underline{v}_{\mu+1} \geqslant 0$. Es sei

$$
\bar{v}_{\infty}=\lim _{\mu \rightarrow \infty} \bar{v}_{\mu} \in H D, \quad \underline{v}_{\infty}=\lim _{\mu \rightarrow \infty} \underline{v}_{\mu} \in H D .
$$

Wir wollen zeigen, dass

$$
\bar{v}_{\infty}=\bigcup_{\nu=1}^{k} u_{\nu}, \quad \underline{v}_{\infty}=\bigcup_{\nu=1}^{k} u_{\nu}
$$

was gleichbedeutend $\mathrm{mit}$

$$
\begin{aligned}
& \bar{v}_{\infty}\left(\varphi\left(e^{i \theta}\right)\right)=\sup _{1 \leqq v \equiv k} u_{\nu}\left(\varphi\left(e^{i 0}\right)\right), \\
& \underline{v}_{\infty}\left(\varphi\left(e^{i \theta}\right)\right)=\inf _{1 \leqq \nu \equiv k} u_{\nu}\left(\varphi\left(e^{i \theta}\right)\right)
\end{aligned}
$$

für fast alle $e^{i 0}$ ist. Es sei ce die Menge jener $e^{i 0}$ für welche

$$
\begin{aligned}
& u_{\nu}\left(\varphi\left(\mathrm{e}^{i 0}\right)\right)=\lim _{\mu \rightarrow \infty} v_{\nu \mu}\left(\varphi\left(e^{i \theta}\right)\right), \quad(\nu=1,2, \ldots, k), \\
& \tilde{v}_{\infty}\left(\varphi\left(e^{i \theta}\right)\right)=\lim _{\mu \rightarrow \infty} \bar{v}_{\mu}\left(\varphi\left(e^{i \theta}\right)\right),
\end{aligned}
$$




$$
\begin{aligned}
& \underline{v}_{\infty}\left(\varphi\left(e^{i 0}\right)^{j}\right)=\lim _{\mu \rightarrow \infty} \underline{v}_{\mu}\left(\varphi\left(e^{i \theta}\right)\right), \\
& \bar{v}_{\mu}\left(\varphi\left(e^{i 0}\right)\right)=\sup _{1 \equiv \nu \geqq k} v_{\nu \mu}\left(\varphi\left(e^{i 0}\right)\right), \quad(\mu=1,2, \ldots) \\
& \underline{v}_{\mu}\left(\varphi\left(e^{i 0}\right)\right)=\inf _{1 \leqq \nu \leqq k} v_{\nu \mu}\left(\varphi\left(e^{i \theta}\right)\right),
\end{aligned}
$$

ist. Sie hat das Mass $2 \pi$. Da die Zahlenfolge $v_{\nu \mu}\left(\varphi\left(e^{i 0}\right)\right)$ mit $/$ abnimmt, so ist für $e^{i \theta} \in(\dot{E}$

$$
\begin{aligned}
& \bar{v}_{\infty}\left(\varphi\left(e^{i \theta}\right)\right)=\lim _{\mu \rightarrow \infty} \bar{v}_{\mu}\left(\varphi\left(e^{i 0}\right)\right)=\lim _{\mu \rightarrow \infty} \sup _{1 \leqq \nu \leqq k} v_{\nu \mu}\left(\varphi\left(e^{i \theta}\right)\right) \geqq \sup _{1 \leqq \nu u_{\nu}} u_{\nu}\left(\varphi\left(e^{i \theta}\right)\right) \\
& \underline{v}_{\infty}\left(\varphi\left(e^{i \theta}\right)\right)=\lim _{\mu \rightarrow \infty} \underline{v}_{\mu}\left(\varphi\left(e^{i \theta}\right)\right)=\lim _{\mu \rightarrow \infty} \inf _{1 \leqq \nu \leqq k} v_{\nu \mu}\left(\varphi\left(e^{i \theta}\right)\right) \geqq \inf _{1 \leqq \nu \leqq k} u_{\nu}\left(\varphi\left(e^{i \theta}\right)\right) .
\end{aligned}
$$

Weiter ist für jedes $\nu_{0}, 1 \leqq \nu_{0} \leqq k$, und $e^{i \theta} \in \mathbb{F}$

$$
\begin{aligned}
& \underline{v}_{\mu}\left(\varphi\left(e^{i \theta}\right)\right)=\inf _{1 \leqq \nu \leqq k} v_{\nu \mu}\left(\varphi\left(e^{i \theta}\right)\right) \leqq v_{\nu_{0} \mu}\left(\varphi\left(e^{i \theta}\right)\right), \\
& \underline{v}_{\infty}\left(\varphi\left(e^{i \theta}\right)\right)=\lim _{\mu \rightarrow \infty} \underline{v}_{\mu}\left(\varphi\left(e^{i \theta}\right)\right) \leqq \lim _{\mu \rightarrow \infty} v_{\nu_{0} \mu}\left(\varphi\left(e^{i \theta}\right)\right)=u_{\nu_{0}}\left(\varphi\left(e^{i \theta}\right)\right)
\end{aligned}
$$

und also

$$
\underline{v}_{\infty}\left(\varphi\left(e^{i \theta}\right)\right) \leqq \inf _{1 \leqq \nu_{0} \leqq k} u_{v_{0}}\left(\varphi\left(e^{i \theta}\right)\right) .
$$

Für $e^{i 0} \in(F$ ist also

$$
\underline{v}_{\infty}\left(\varphi\left(e^{i \theta}\right)\right)=\inf _{1 \leqq} u \cdot\left(\varphi\left(e^{i \theta}\right)\right) .
$$

Es sei $\nu_{\mu}$ jenes $\nu, 1 \leqq \nu_{\mu} \leqq k$, für welches

$$
v_{\nu \mu \mu}\left(\varphi\left(e^{i \theta}\right)\right)=\sup _{1 \leqq \vdots \triangleq k} v_{\nu \mu}\left(\varphi\left(e^{i \theta}\right)\right)
$$

ist. $\mathrm{Da} \nu_{k}$ nur endlich viele Werte annehmen kann, so ist für unendlich viele $\mu, \mu \in J, \nu_{\mu}=\nu_{0}$ und es ist

$$
u_{\nu_{0}}\left(\varphi\left(e^{i \theta}\right)\right)=\lim _{J \exists \mu \rightarrow \infty} v_{\nu_{0} \mu}\left(\varphi\left(e^{i \theta}\right)\right)=\lim _{J \exists: \lambda \rightarrow \infty} \sup _{1 \leqq \nu \leqq k} v_{\nu \mu}\left(\varphi\left(e^{i \theta}\right)\right)=\lim _{\mu \rightarrow \infty} \bar{v}_{\mu}\left(\varphi\left(e^{i \theta}\right)\right)=\bar{v}_{\infty}\left(\varphi\left(e^{i \theta}\right)\right)
$$

woraus

$$
\bar{v}_{\infty}\left(\varphi\left(e^{i \vartheta}\right)\right) \leqq \sup _{1 \leqq \nu \equiv k} u_{\nu}\left(\varphi\left(e^{i \vartheta}\right)\right)
$$

und

$$
\bar{v}_{\infty}\left(\varphi\left(e^{i 0}\right)\right)=\sup _{1 \leqq \nu \triangleq k} u_{\nu}\left(\varphi\left(e^{i \theta}\right)\right)
$$

für $e^{i 0} \in(\xi$ folgt. Damit ist der Beweis ausgeführt.

19. Satz 10. Wenn $M$ eine maximale HD-unteilbare Menge ist, so ist $\omega(z, M, R)$ eine in der Klasse HD minimale Funktion. Ist $u(z)$ eine minimale 
Funktion in der Klasse HD (oder $H D)$, so ist $u(z)=c \omega(z, M, R)$ und $M$ ist eine maximale HD.unteilbare Menge.

Es sei $u(z)$ eine minimale Funktion in der Klasse $H D$ (oder $H D$ ) und sei $u_{N}(z)$ die untere Hülle von $u(z)$ und $N$. Nach dem Hilfssatz 6 ist $u_{x}(z) \in H D$ (oder $H D$ ), und da $u_{v}(z) \leqq u(z)$ ist, so folgt $u_{v}(z)=c_{*} u(z)$. Aus

$$
u(\varphi(t))=\int_{0}^{2 \pi} u\left(\varphi\left(e^{i 0}\right)\right) P(\theta, t) d \theta
$$

sieht man, dass $u\left(\varphi\left(e^{i n}\right)\right)$ nicht fast überall Null ist. Daraus folgt, dass ein $N$ existiert, so dass $u_{x}(z) \neq 0$ und $c_{x} \neq 0$ ist. $u(z)$ ist also beschränkt.

Es sei

$$
c=\sup _{z \in R} u(z)
$$

und $\bar{u}(z)$ die obere Hülle von $u(z)$ und $c^{\prime}, c^{\prime}<c$ :

$$
\left.\bar{u}(\varphi(t))=\int_{0}^{2 \pi} \sup \left(u\left(\varphi\left(e^{i \theta}\right)\right), c^{\prime}\right) P(\theta, t) d \theta \in \underline{H D} \quad \text { (oder } H D\right)
$$

Wir stellen

$$
\bar{u}_{0}(z)=c \frac{\bar{u}(z)-c^{\prime}}{c-c^{\prime}} \in H D \quad(\text { oder } H D)
$$

Es ist $\bar{u}_{0}\left(\varphi\left(e^{i \theta}\right)\right)=0$ fast überall wo $u\left(\varphi\left(e^{i \theta}\right)\right) \leqq c^{\prime}$, und $\bar{u}_{0}\left(\varphi\left(e^{i \theta}\right)\right) \leqq u\left(\varphi\left(e^{i \theta}\right)\right)$ fast überall wo $u\left(\varphi\left(e^{i \theta}\right)\right) \geqslant c^{\prime}$ ist. Es ist also $\bar{u}_{0}(z) \leqq u(z)$ und, da $u(z)$ minimal ist, ist $\bar{u}_{0}(z)=c_{0} u(z)$. Es ist also $u\left(\varphi\left(e^{i 0}\right)\right)=0$ fast überall wo $u\left(\varphi\left(e^{i 0}\right)\right) \leqq c^{\prime}$ ist und, da $c^{\prime}$ beliebig war, $c^{\prime}<c$, so ist $u\left(\varphi\left(e^{i \theta}\right)\right)$ fast überall entweder Null oder gleich $c$ und $u(z)=c \omega(z, M, R)$.

Nun bleibt $z \mathrm{u}$ zeigen, dass $M$ eine maximale $H D$-unteilbare Menge ist. Nach dem Folgesatz 1 genügt es zu zeigen, dass sie eine $H B D$-unteilbare Menge ist. Es sei $0 \leqq v(z) \in H B D$ und

$$
c_{0}=\sup _{z \in R} v(z) .
$$

Wir werden die untere Hülle $\underline{v}(z)$ von $v(z)$ und $2 c_{0} \omega(z, M, R)$ betrachten. Fast überall auf $\mathfrak{M}$ ist $\underline{v}\left(\varphi\left(e^{i \theta}\right)\right)$ gleich $v\left(\varphi\left(e^{i \theta}\right)\right)$. Da

$$
\underline{v}(z) \leqq 2 c_{0} \omega(z, M, R), \quad \underline{v}(z) \in \underline{H D} \quad(\operatorname{oder} H D)
$$

ist und da $\omega(z, M, R)$ minimal in der Klasse $H D$ (oder $H D$ ) ist, so folgt $\underline{v}(z)=\underline{c} \omega(z, M, R)$. Auf $\mathfrak{M}$ ist also fast überall 


$$
v\left(\varphi\left(e^{i n}\right)\right)=\underline{v}\left(\varphi\left(e^{i 0}\right)\right)=\underline{c}
$$

und $M$ ist eine $H D$-unteilbare Menge.

$\mathrm{Da} \omega(z, M, R) \in \underline{H D}$, so gibt es eine Folge $\left\{u_{\nu}(z)\right\}$ für die

$$
\begin{aligned}
& \omega(z, M, R)=\lim _{\nu \rightarrow \infty} u_{\nu}(z), \\
& u_{\nu}(z) \geqslant u_{\nu+1}(z) \geq 0, \quad u_{\nu}(z) \in H D
\end{aligned}
$$

ist. Wäre $\bar{M}$ eine $H D$-unteilbare Menge $\bar{M} \supseteqq M$ und

$$
\omega(z, \bar{M}-M, R)>0,
$$

so würde $\imath_{\imath}\left(\varphi\left(e^{i}\right)\right)=c_{\nu} \geqslant 1$ für fast alle $e^{i \theta} \in \overrightarrow{\mathfrak{M}}$ sein. Daraus folgt

$$
\omega\left(\varphi\left(e^{i \theta}\right), M, R\right)=\lim _{\nu \rightarrow \infty} c_{\nu}=1
$$

für fast alle $e^{i \theta} \in \overline{\mathfrak{M}}$ im Widerspruch zu der Definition von $\omega(z, M, R) . \quad M$ ist also eine maximale $H D$-unteilbare Menge und der zweite Teil des Satzes ist bewiesen.

Wir beweisen jetzt den ersten Teil. Es sei $M$ eine maximale $H D$-unteilbare Menge. Wir bezeichnen mit $\mathfrak{u}_{u}$ die Menge jener Funktionen $u(z) \in H D$

$$
0<u(z) \leqq 1,
$$

für welche $u\left(\varphi\left(e^{i \theta}\right)\right)=1$ fast überall auf $\mathfrak{M}$ ist, und es sei

$$
v_{0}(z)=\inf _{u \in \mathfrak{U} M} u(z) .
$$

Es sei $v_{n}(z) \in \mathfrak{U}_{M}$ so, dass

$$
\lim _{n \rightarrow \infty} v_{n}\left(z_{0}\right)=v_{0}\left(z_{0}\right)
$$

Wenn wir

$$
\underline{v}_{n}=\bigcap_{\nu=1}^{n} v_{\nu} \in H D
$$

nehmen, so ist

$$
\begin{aligned}
& \underline{v}_{n}(z) \geq \underline{v}_{n+1}(z) \geq 0, \\
& \underline{v}_{0}(z)=\lim _{n \rightarrow \infty} \underline{v}_{n}(z) \in H D, \quad \underline{v}_{0}\left(z_{0}\right)=v_{0}\left(z_{0}\right) .
\end{aligned}
$$

Für einen zweiten Punkt $z_{1}$ sei $v_{n}^{\prime} \in \mathfrak{U}_{M}$ so, dass

$$
\lim _{n \rightarrow \infty} v_{n}^{\prime}\left(z_{1}\right)=v_{0}\left(z_{1}\right) \text { und } \underline{v}_{n}^{\prime}=\underline{v}_{n} \cap\left(\bigcap_{\nu=1}^{n} v_{\nu}^{\prime}\right) \text {. }
$$


Dann ist

$$
\begin{aligned}
& \underline{v}_{n}^{\prime}(z) \leqq \underline{v}_{n}(z), \quad \underline{v}_{n}^{\prime}(z) \geqq v_{n+1}^{\prime}(z), \\
& \underline{v}_{0}^{\prime}(z)=\lim _{n \rightarrow \infty} \underline{v}_{n}^{\prime}(z) \leqq \lim _{n \rightarrow \infty} \underline{v}_{n}(z)=\underline{v}_{0}(z), \\
& \underline{v}_{0}^{\prime}\left(z_{1}\right)=v_{v}\left(z_{1}\right) .
\end{aligned}
$$

Aus $v_{0}\left(z_{0}\right) \leqq \underline{v}_{0}^{\prime}\left(z_{0}\right) \leqq \underline{v}_{0}\left(z_{0}\right)=v_{0}\left(z_{0}\right)$ folgt $\underline{v}_{0}^{\prime}\left(z_{0}\right)=\underline{v}_{0}\left(z_{0}\right)$ und daher, aus dem Maximumprinzip für harmonische Funktionen $\underline{v}_{0}^{\prime}(z) \equiv \underline{v}_{0}(z)$ und $v_{0}\left(z_{1}\right)=\underline{v}_{0}\left(z_{1}\right)$. $\mathrm{Da} z_{1}$ beliebig war, so ist

$$
v_{0}(z) \equiv \underline{v}_{0}(z) \in \underline{H D} .
$$

Fast überall auf $\mathfrak{M}$ ist $v_{0}\left(\varphi\left(e^{i 0}\right)\right)$ gleich 1 . Es sei $V_{1}(z)$ die obere Hülle von $v_{0}(z)$ und $C_{1}<1$. Dann ist

$$
V_{2}(z)=\frac{V_{1}(z)-C_{1}}{1-C_{1}} \in \underline{H D}
$$

und man sieht, genau wie oben, dass $V_{2}\left(\varphi\left(e^{i \theta}\right)\right)=0$ fast überall wo $v_{0}\left(\varphi\left(e^{i \theta}\right)\right) \leqq C_{1}$ ist, und dass $V_{2}(z) \leqq v_{0}(z)$ ist. $\mathrm{Da} V_{2}\left(\varphi\left(e^{i \theta}\right)\right)=1$ fast überall auf $\mathfrak{M}$ ist und, da sie als Grenzfunktion einer abnähmenden Folge von Funktionen aus $H D$ dargestellt werden kann, so sieht man leicht aus dar Definition von $v_{0}(z)$, dass $v_{0}(z) \leqq V_{2}(z)$ ist. Daraus ergibt sich $v_{0}(z)=V_{2}(z)$ und $v_{0}\left(\varphi\left(e^{i 0}\right)\right)$ ist gleich Null fast überall wo $v_{0}\left(\varphi\left(e^{i 0}\right)\right)$ kleiner als $C_{1}$ ist. Da $C_{1}<1$ beliebig war, so folgt, dass $v_{0}(\varphi(t))$ fast überall auf einer Menge $\overline{\mathfrak{M}} \supseteqq \mathfrak{M}_{i}$ den Winkelgrenzwert 1 hat, und fast überall auf $C \bar{M}$ den Winkelgrenzwert 0 hat.

Wir nehmen an, dass $\overline{\mathfrak{M}}-\mathfrak{M}$ positives, Lebesguesches Mass hat. Da $M$ eine maximale $H D$-unteilbare Menge war, so kann man eine Funktion $V_{3}(z) \in H B D$ finden, für die $V_{3}\left(\varphi\left(e^{i \theta}\right)\right)=C_{3}$ fast überall auf $\mathfrak{M}$ und $V_{3}\left(\varphi\left(e^{i \theta}\right)\right) \neq C_{3}$ fast überall auf einer Menge $\overline{\mathfrak{M}} \leqq \overline{\mathfrak{M}}-\mathfrak{M}$ von positiven Mass ist. Wir bezeichnen mit $\bar{V}_{3}(z)$ bzw. $V_{3}(z)$ die obere bzw. untere Hülle von $V_{3}(z)$ und $C_{3}$. Die Funktion $V_{4}(z)$ $=\bar{V}_{3}(z)-\underline{V}_{3}(z)$ gehört der Klasse $H B D$ und ist nichtnegativ. Fast überall auf $\mathfrak{N}$ ist $V_{\downarrow}\left(\varphi\left(e^{i \theta}\right)\right)=0$ und fast überall auf $\overline{\mathfrak{M}}$ ist $V_{\mathfrak{\downarrow}}\left(\varphi\left(\mathrm{e}^{i 0}\right)\right)>0$. Es sei

$$
C_{4}=\sup _{z \in R} V_{4}(z)
$$

und

$$
V_{5}(z)=\frac{C_{4}-V_{1}(z)}{C_{4}}
$$

Wir haben $V_{5}(z) \in H D, 0<V_{5}(z) \leqq 1$ und fast überall auf $\mathfrak{M}$ ist $V\left(\varphi\left(e^{i 0}\right)\right)=1$ : 
Daraus folgt $V_{5}(z) \in \mathfrak{U}_{M}$ und also $v_{0}(z) \leqq V_{5}(z)$. Auf $\overline{\mathfrak{M}}$ ist $V_{5}\left(\varphi\left(e^{i 0}\right)\right)$ fast überall kleiner als $1 \mathrm{im}$ Widerspruch mit der Tatsache, dass $v_{0}\left(\varphi\left(e^{i 0}\right)\right)$ fast überall gleich 1 ist. Daraus folgt, dass $\overline{\mathfrak{M}}-\mathfrak{M}$ nulles Mass hat und es ist

$$
v_{0}(z)=\omega(z, M, R) \in H D .
$$

Es ist noch zu zeigen, dass $\omega(z, M, R)$ minimal in der Klasse $H D$ ist, Dafür sei $V_{6}(z) \in H D$ und $0 \leqq V_{6}(z) \leqq \omega(z, M, R)$. Dann ist $V_{6}\left(\varphi\left(e^{i \theta}\right)\right)=0$ fast überall auf $\left.C^{\jmath}\right\}$. $\mathrm{Da} V_{6}(z) \in \underline{H D}$, so gibt es eine Folge $\left\{V_{6 v}(z)\right\}$ für die

$$
\begin{aligned}
& V_{6}(z)=\lim _{\nu \rightarrow \infty} V_{6 \nu}(z), \\
& V_{6 \nu}(z) \geqq V_{6, \nu+1}(z) \geqq 0, \quad V_{6 \nu}(z) \in H D
\end{aligned}
$$

ist. Da $M$ eine $H D$-unteilbare Menge ist, so ist $V_{6 v}\left(\varphi\left(e^{i 0}\right)\right)=C_{6}$, fast überall auf $\mathfrak{M}$. Ferner ist

$$
V_{6}\left(\varphi\left(e^{i 0}\right)\right)=\lim _{\nu \rightarrow \infty} V_{6 \nu}\left(\varphi\left(e^{i 0}\right)\right)=\lim _{\nu \rightarrow \infty} C_{6 \nu}=C_{6}
$$

fast überall auf $\mathfrak{M}$. Daraus ergibt sich $V_{6}(z)=C_{6} \omega(z, M, R)$ und $\omega(z, M, R)$ ist eine minimale Funktion in der Klasse $H D$. Damit ist der Beweis des Satzes $10 \mathrm{zu}$ Ende geführt.

Der zwischen $H B$ und $H D$ existierende Parallelismus ist durch den Satz 10 nicht verletzt. Nur scheint bei $H B$ die Beziehung zwischen den minimalen Funktionen und den $H B$-unteilbaren Mengen einfacher zu sein, weil $H B$ mit $H B$ zusammenfällt; deshalb sind die in der Klasse $H B$ minimalen Funktion in der Klasse $H B$ enthalten.

Folgesatz 2. Jede in der Klasse HD (bzw. HD) minimale Funktion ist beschränkt.

20. Hilfssatz 7. Es sei $R$ eine Riemannsche Fläche und $R^{\prime}$ ein Gebiet auf $R$ mit analytischem Rande. Ist $M^{\prime}$ eine HD-unteilbare Menge auf dem idealen Rande von $R^{\prime}$, so ist $M=E^{*} M^{\prime}$ eine HD-unteilbare Menge auf dem idealen Rande von $R$.

$\mathrm{Da}$ jede $H B D$-unteilbare Menge eine $H D$-unteilbare Menge ist, so genügt zu beweisen, dass für jede Funktion $u(z) \in H D$ auf $R, 0 \leqq u(z) \leqq 1, u(\zeta(t))$ fast überall auf $\mathfrak{M}$ einen konstanten Winkelgrenzwert besitzt. Da $u(z)$ auch auf $R^{\prime}$ endliches Dirichlet-Integral hat, so hat $u\left(c^{\prime}\left(t^{\prime}\right)\right)$ fast überall auf $\mathfrak{D}^{\prime}$ einen konstanten Winkelgrenzwert: $u\left(\varphi^{\prime}\left(e^{i 0^{\prime}}\right)\right)=c$ für fast alle $e^{i 0^{\prime}} \in \mathfrak{M} \mathfrak{i}^{\prime}$. Aus 11 . 
folgt dann $\operatorname{Iu}\left(c^{\prime}\left(e^{i 0^{\prime}}\right)\right)=c$ für fast alle $e^{i 0^{\prime}} \equiv \mathfrak{N}^{\prime}$. Auf $R^{\prime}$ ist offenbar

$$
c \omega\left(z, M^{\prime}, R^{\prime}\right) \leqq I u(z) \leqq c \omega\left(z, M^{\prime}, R^{\prime}\right)+\omega\left(z, M_{1}^{\prime}-M^{\prime}, R^{\prime}\right)
$$

und daher

$$
\begin{aligned}
& c E_{\omega}\left(z, M^{\prime}, R^{\prime}\right) \leqq E I u(z) \leqq c E \omega\left(z, M^{\prime}, R^{\prime}\right)+E \omega\left(z, M_{1}^{\prime}-M^{\prime}, R^{\prime}\right), \\
& c \omega(\varphi(t), M, R) \leqq E I u(z) \leqq c \omega(\varphi(t), M, R)+\omega\left(\varphi(t), M_{1}-M, R\right),
\end{aligned}
$$

Nun sieht man aus der letzten Ungleichung, dass

$$
\operatorname{EIu}\left(\varphi\left(e^{i \theta}\right)\right)=c
$$

fast überall auf $\mathfrak{M}$ und, da auf $\mathfrak{M}, u\left(\varphi\left(e^{i 0}\right)\right)$ und $E I u\left(\varphi\left(e^{i 0}\right)\right)$ für fast alle $e^{i 0}$ denselben Winkelgrenzwert haben, so ist $u\left(c\left(e^{i \theta}\right)\right)=c$ für fast alle $e^{i 0} \in \mathfrak{M}$, was $\mathrm{zu}$ beweisen war.

Wir sagen, dass eine Menge $M^{\prime}$ auf dem idealen Rande von $R^{\prime}$ und von positivem Masse an den relativen Rand $\partial R^{\prime}$ von $R^{\prime}$ gebunden ist, wenn für jede Funktion $u(z) \in H D$, die auf $\partial R^{\prime}$ verschwindet, $u\left(\varphi^{\prime}\left(t^{\prime}\right)\right)$ fast überall auf $\mathfrak{M}^{\prime}$ den Winkelgrenzwert Null hat. Im entegengesetzten Falle sagen wir, dass die Menge $M^{\prime}$ frei in Bezug auf $\partial R^{\prime}$ ist. Ist z.B. $R^{\prime}=R-K$, wo $K$ eine Kompakte Menge ist, so ist jedes $M^{\prime}=I^{*} M$ frei.

Es sei $M$ eine $H D$-unteilbare Menge auf dem Rande von $R$ und $R^{\prime}$ ein Gebiet mit analytischem Rande auf $R$. Hat $u^{\prime}(z) \in \mathfrak{u}^{\prime}$ auf $R^{\prime}$ ein endliches Dirichlet-Integral, so hat auch $E u^{\prime}(z)$ auf $R$ ein endliches Dirichlet-Integral, denn es ist

$$
D_{R}\left(E u^{\prime}\right) \leqq \lim _{n \rightarrow \infty} D_{R_{\mu}}\left(E_{n} u^{\prime}\right) \leqq \lim _{n \rightarrow \infty} D_{R_{u} \cap R^{\prime}}\left(u^{\prime}\right)=D_{R^{\prime}}\left(u^{\prime}\right)<\infty
$$

Auf $\mathfrak{M}$ hat somit $E u^{\prime}(\varphi(t))$ fast überall einen konstanten Winkelgrenzwert $c$, und daher ist auf $R$

$$
c \omega(z, M, R) \leqq E u^{\prime}(z) \leqq c \omega(z, M, R)+c^{\prime} \omega\left(z, M_{1}-M, R\right)
$$

wo

$$
c^{\prime}=\sup _{z \in R^{\prime}} u^{\prime}(z)
$$

ist. Daraus folgt

$$
c \omega\left(z, M^{\prime}, R^{\prime}\right) \leqq u^{\prime}(z) \leqq c \omega\left(z, M^{\prime}, R^{\prime}\right)+c^{\prime} \omega\left(z, M_{1}^{\prime}-M^{\prime}, R^{\prime}\right)
$$

wo $M^{\prime}=I^{*} M$ ist ; daraus kann man sehen, dass $\iota^{\prime}\left(\varphi^{\prime}\left(e^{i \theta^{\prime}}\right)\right)=c$ fast überall auf $\mathfrak{M}^{\prime}$ ist. Also nehmen alle Funktionen aus $\mathfrak{U}^{\prime}$, welche ein endliches Dirichlet- 
Integral auf $R^{\prime}$ haben, fast überall auf $\mathfrak{M}^{\prime}$ einen konstanten Winkelgrenzuert.

Wir nehmen jetzt an, dass $M^{\prime}$ frei in Bezug auf $\partial R^{\prime}$ ist und zeigen, dass sie eine $H D$-unteilbare Menge ist. Sei $u(z)$ eine positive und beschränkte $H D$ Funktion auf $R^{\prime}$. Da $M^{\prime}$ frei ist, so existiert eine $H D$-Funktion $u_{0}(z)$ auf $R^{\prime}$, welche auf $\partial R^{\prime}$ verchwindet und so, dass $u_{j}\left(c^{\prime}\left(e^{i \eta^{\prime}}\right)\right)$ nicht gleich Null für fast alle $e^{i 0^{\prime}} \in \mathfrak{M}^{\prime}$ ist. Nach Obigem ist $u_{0}\left(\varphi^{\prime}\left(e^{i 0^{\prime}}\right)\right)=c_{0}>0$ für fast alle $e^{i 0^{\prime}} \in \mathfrak{M}^{\prime}$. Es sei

$$
c=\sup _{z \in R^{\prime}} u(z)
$$

und $\underline{u}(z)$ die untere Hülle von $u(z)$ und $\frac{2 c}{c_{0}} u_{0}(z)$. $\underline{u}(z)$ verschwindet auf $\partial R^{\prime}$ und es ist $\underline{u}\left(\varphi^{\prime}\left(e^{i \theta^{\prime}}\right)\right)=u\left(\varphi^{\prime}\left(e^{i 0^{\prime}}\right)\right)$ für fast alle $e^{i \theta^{\prime}} \in \mathfrak{M}^{\prime}$. Da $\underline{u}(z) \in H D$ und auf $\partial R^{\prime}$ verschwindet, so folgt, dass fast überall auf $\mathfrak{M}^{\prime} \underline{u}\left(\varphi^{\prime}\left(e^{i 0^{\prime}}\right)\right)=c^{\prime}$ ist und dasselbe gilt für $u\left(c^{\prime}\left(e^{i \emptyset^{\prime}}\right)\right) . M^{\prime}$ ist somit $H D$-unteilbar.

Hilfssatz 8. Es sei $R$ eine Riemannsche Fläche und $R^{\prime}$ ein Gebiet init analytischem Rande auf $R$. Ist $M$ eine HD-unteilbare Menge in Rande von $R$ und ist $I^{*} M=M^{\prime}$ frei in Bezug auf $\partial R^{\prime}$, so ist $M^{\prime}$ eine HD-unteilbare Menge.

Es ist uns nicht gelungen $\mathrm{zu}$ beweisen, dass $M^{\prime}$ immer eine $H D$-unteilbare Menge ist. Auch konnten wir kein entsprechendes Gegenbeispiel bilden.

Es sei $u(z)$ eine beschränkte harmonische Funktion mit endlichem DirichletIntegral auf $R$ und $M$ sei die $H D$-unteilbare Menge im Rande von $R . \quad$ Es ist $u\left(\varphi\left(e^{i \theta}\right)\right)=c$ für fast alle $e^{i 0} \in \mathfrak{M}$. Falls

$$
\sup _{z \in d R^{\prime}} u(z)=c^{\prime}<c
$$

ist, so ist die Menge $M^{\prime}=I^{*} M$ frei in Bezug auf $\partial R^{\prime}$, wenn sie positives Mass hat. Denn $u(z)$ gehört der Klasse $H D$ auf $R^{\prime}$ an und ebenso die obere Hülle $\bar{u}(z)$ von $u(z)$ und $c^{\prime}$. $\bar{u}(z)-c^{\prime}$ ist gleich Null auf $\partial R^{\prime}$ und es ist

$$
\bar{u}\left(\varphi^{\prime}\left(e^{i \theta^{\prime}}\right)\right)-c^{\prime}=c-c^{\prime}>0
$$

für fast alle $e^{i \theta^{\prime}} \in \mathfrak{M} \ell^{\prime}$, wie man leicht sehen kann, woraus die Behauptung entspringt.

Ist $M$ eine $H D$-unteilbare Menge und sind $M \cap M_{0}, \quad M \cap M_{1}$ von positivem Masse, so ist $M^{\prime}=I^{*} M$ an den Rand $\partial R^{\prime}$ von $R^{\prime}$ gebunden. Denn sei $u^{\prime}(z) \in \mathfrak{u}^{\prime}$ mit beschränktem Dirichlet-Integral; dann hat auch $E u^{\prime}(z)$ ein beschränktes Dirichlet-Integral und es ist $E \mathfrak{u}^{\prime}\left(\varphi\left(e^{i \theta}\right)\right)=0$ fast uiberall auf $\mathfrak{M}^{\prime} \cap \mathfrak{M}_{0}$. Da $M$ 
eine $H D$-unteilbare Menge ist, so ist $E u^{\prime}\left(\varphi\left(e^{i 0}\right)\right)=0$ fast überall auf $\mathfrak{M}$ und daraus folgt $u^{\prime}\left(c^{\prime}\left(e^{i 0^{\prime}}\right)\right)=0$ fast überall auf $\mathfrak{M} \mathfrak{X}^{\prime} . M^{\prime}$ ist somit gebunden.

\section{Einige Anwendungen und Beispiele}

21. SATZ 11. Jede Fortsetzung (eigentliche oder uneigentliche [16] Seite 245) einer Riemannschen Fläche aus der Klasse $U_{H B}$ (bzw. $\left.U_{H D}\right)$ gehört auch dev Klasse $U_{H B}\left(b z w . U_{H D}\right)$ an.

Es sei $\underline{R}$ die Fortsetzung von $R \in U_{H Y}(Y=B, D)$ und $M$ sei die $H Y$ unteilbare Menge auf dem idealen Rande von $R$. Wir bezeichnen mit $\Delta$ die offene Menge von $R$, wo $\omega(z, M, R)>\varepsilon>0$ ist. Der Rand von $\Delta$ ist analytisch und wir bezeichnen mit $E, I$ die Operatoren in Bezug auf das Paar $(\Delta, R)$. Es sei $\xi(z)$ gleich 1 in $\Delta$ und gleich 0 in $R-\Delta$ und $e^{i 0}$ ein Punkt, wo $\omega\left(\varphi\left(e^{i \theta}\right), M, R\right)$ $=1$ ist. Auf jeder Kurve $\lambda$, die in $e^{i 0}$. einen Winkeleingang hat, konvergiert $\omega(\varphi(t), M, R)$ gegen 1 und deshalb konvergiert auch $\xi(\varphi(t))$ auf $\lambda$ gegen 1 . $\xi(\varphi(t))$ hat somit in $e^{i 0}$ den Winkelgrenzwert 1 . Ähnlicherweise beweist man, dass $\xi(\varphi(t))$ in $e^{i \theta}$ den Winkelgrenzwert 0 hat, wenn $\omega\left(\varphi\left(e^{i \theta}\right), M, R\right)=0$ ist. Daraus folgt, dass $\xi\left(\varphi\left(e^{i 0}\right)\right)$ gleich 1 fast überall auf $\mathfrak{M}$ und gleich 0 fast überall auf $C \mathfrak{M}$ ist. Mit den Bezeichnungen von 13. ist also

$$
\mathfrak{M}=\mathfrak{M}_{1}^{*}, \quad C \mathfrak{M}=\mathfrak{M}_{0}^{*},
$$

woraus $M=M_{1}$ folgt. Es ist also $\operatorname{EI} 1(z)=\omega(z, M, R)$.

$\Delta$ ist zu gleicher Zeit eine offene Menge auf $\underline{R}$ und sein relativer Rand besteht aus dem relativen Rande von $\Delta$ in Bezug auf $R, \partial_{R} \Delta$, und aus Punkten des relativen Randes von $R$ in Bezug auf $\underline{R}, \partial_{R} R$. Die lezten zerfallen in zwei Teile, je nachdem sie Häufungspunkte von $\partial_{R} \Delta$ sind oder nicht. Der zweite Teil $K_{0}$ ist eine Menge von der Kapazität Null (d.h. ihr Durchschnitt mit jeder Parameterumgebung ist von der Kapazität null). Im entgegengesetzten Falle kann man $K_{0}$ in zwei Mengen $K_{1}, K_{2}$ von positiver Kapazität teilen, und sei $u_{\imath}^{\prime}(z)$ das harmonische Mass von $K_{\nu}$ in Bezug auf $\Delta$. Es ist offenbar

$$
\begin{aligned}
& \sup _{z \in \Delta} u_{:}^{\prime}(z)=1, \quad 0<u_{1}^{\prime}(z)+u_{2}^{\prime}(z) \leqq I 1(z), \\
& u_{\nu}^{\prime}(z) \in \mathfrak{U}^{\prime}, \quad D_{\Delta}\left(u_{\nu}^{\prime}\right)<\infty
\end{aligned}
$$

Daraus folgt 


$$
\begin{aligned}
& \sup _{z \in R} E u_{\nu}^{\prime}(z)=1, \\
& E u_{1}^{\prime}(z)+E u_{2}^{\prime}(z) \leqq E I 1(z)=\omega(z, M, R), \\
& D_{R}\left(E u_{\nu}^{\prime}\right)<\infty .
\end{aligned}
$$

Da nach dem Satz 3 (bzw. Satz 10) $\omega(z, M, R$ ) minimal in der Klasse $H B$ (bzw. HD) ist, so ist

$$
E u_{\nu}^{\prime}(z)=c_{\nu} \omega(z, M, R)
$$

Es ist aber

$$
1=\sup _{z \in R} E u_{z}^{\prime}(z)=c_{\nu} \sup _{z \in R} \omega(z, M, R)=c_{\nu}
$$

und

$$
c_{1} \omega(z, M, R)+c_{2} \omega(z, M, R)=E u_{1}^{\prime}(z)+E u_{2}^{\prime}(z) \leqq \omega(z, M, R),
$$

was widersinnig ist. Da $\varepsilon>0$ beliebig ist, so besteht der ganze Rand von $\Delta$ in Bezug auf $R$, $\partial_{R} \Delta$, aus $\partial_{R} \Delta$ und einer Menge von der Kapazität Null. Deshalb kann man auch in Bezug auf das $\operatorname{Paar}(\Delta, \underline{R})$ die Operatoren $\underline{E}$ und $I$ bilden.

Wir wollen erst beweisen, dass $\underline{R} \notin O_{G}$. Deshalb sei $K$ eine Kreisscheibe auf $R-\Delta$, und $\left\{\underline{R}_{n}\right\}$ eine Ausschöpfung von $\underline{R}$, so dass $\partial \underline{R}_{n}$ aus endlich vielen analytischen Jordankurven besteht. Wir bezeichnen mit $\omega_{n}(v)$ die harmonische Funktion in $\underline{R}_{n}-\bar{K}$, welche auf $\underline{R}_{n}-K$ stetig ist, auf $\partial K$ gleich 0 und auf $\partial \underline{R}_{n}$ gleich 1 ist. Der Rand von $\underline{R}_{n} \cap \Delta$ besteht aus $\partial_{R} \Delta \cap \underline{R}_{n}, \partial \underline{R}_{n} \cap \Delta$ und einer Menge von der Kapazität Null. $\omega_{n}(z)$ ist in $R_{n} \cap \Delta$ harmonisch und auf $\partial_{R} A \cap \underline{R}_{n}$ und $\partial \underline{R}_{n} \cap \Delta$ ist $\omega(z, M, R)-\varepsilon \leqq \omega_{n}(z)$. Deshalb besteht dieselbe Ungleichung in $\underline{R}_{n} \cap A$, und für $n \rightarrow \infty$ ist

$$
0<\omega(z, M, R)-\varepsilon \leqq \lim _{n \rightarrow \infty} \underline{\omega}_{n}(z)
$$

Daraus folgt, dass $\underline{R}$ eine positiv berandete Riemannsche Fläche ist.

Es sei jetzt $u(z)$ eine positive beschränkte harmonische Funktion (und im Falle $Y=D$ auch mit beschränktem Dirichlet-Integral) auf $R . \quad u(z)$ besitzt zu gleicher Zeit dieselben Eigenschaften auf $R$ und, da $M$ eine $H Y$ unteilbare Menge ist, so hat $u(\varphi(t))$ fast überall auf $\mathfrak{M}$ einen konstanten Winkelgrenzwert $c^{\prime}$. Wenn

$$
c=\sup _{z \in R} u(z)
$$

ist, so ist

$$
c^{\prime} \omega(z, M, R) \leqq u(z) \leqq c^{\prime} \omega(z, M, R)+c \omega(z, F-M, R)
$$


Da aber $M=M_{1}$ ist, so ist $I \omega(z, F-M, R)=0$

und daraus folgt $I \boldsymbol{u}(z)=c^{\prime} I \omega(z, M, R)$.

Es ist aber

$$
I u(z)=\underline{I} u(z), \quad I \omega(z, M, R)=I 1(z)=\underline{I} 1(z)
$$

und also $\underline{I} u(z)=c^{\prime} \underline{I} 1(z)$.

Daher ergibt sich

$$
\underline{E} \underline{I} u(z)=c^{\prime} \underline{E} \underline{I} 1(z)=c^{\prime} \omega\left(z, \underline{M}_{1}, \underline{R}\right) .
$$

Es sei $\underline{\underline{c}}(\underline{t})$ die eineindeutige und konforme Abbildung des Kreises $|\underline{t}|<1$ auf die universelle Überlagerungsfläche von $\underline{R}$ und $\underline{\Re}_{1}$ das Bild der Menge $\underline{M}_{1}$. Nach 11. haben $u(\underline{\varphi}(\underline{t}))$ und $\underline{E} \underline{I} u(\underline{\varphi}(\underline{t}))$ fast überall auf $\underline{\mathfrak{j}}_{1}$ dieselben Winkelgrenzwerte. Daraus folgt, dass $u(\underline{\varphi}(\underline{t}))$ fast überall auf $\mathfrak{M}_{1}$ den konstanten Winkelgrenzwert $c^{\prime}$ hat und $\underline{M}_{1}$ ist eine $H Y$-unteilbare Menge. Die Riemannsche Fläche $\underline{R}$ gehört somit der Klasse $U_{H Y}$ an, was zu beweisen war.

Wir sagen von einer abgeschlossenen (nicht unbedingt kompakten) Menge $K \subset R$, dass sie dïnn ist, wenn man eine abgeschlossene Menge $K^{*} \supseteqq K$ finden kann, so dass die offene Menge $\Delta=R-K^{*}$ einen analytischen Rand hat dun $E I 1=1$ ist, wo die Operatoren $E$ und $I$ in Bezug auf das Paar $(\triangle, R)$ zu nehmen sind.

So zum Beispiel ist jede Kompakte Menge dünn, denn es gilt das allgemeine Kriterium : Ist

$$
\inf _{z \in K} g\left(z, z_{0}\right)=\varepsilon_{K}>0,
$$

so ist die Menge $K$ dünn. Es sei $K^{*}$ die Menge $g\left(z, z_{0}\right) \geqq \varepsilon_{K}$. Dann ist $K \leqq K^{*}$ und $K^{*}$ hat analytischen Rand. Wir stellen $\xi(z)=1$ auf $\Delta=R-K^{*}$ und $\xi(z)=0$ auf $K^{*}$. Es sei $e^{i 0}$ ein Punkt der Menge $F$ und $\lambda$ eine Kurve im Kreise $|t|>1$, die in $e^{i \theta}$ einen Winkeleingang hat. Auf $\lambda$ konvergiert $g\left(\varphi(t), z_{0}\right)$ gegen Null, woraus $z$ u ersehen ist, dass $\xi(\varphi(t))$ auf $\lambda$ gegen 1 konvergiert. Da $\lambda$ beliebig war, so folgt, dass $\xi(\varphi(t))$ in $e^{i \theta}$ den Winkelgrenzwert 1 hat. Es ist also $e^{i 0} \in \mathfrak{M}_{1}^{*}$, für jedes $e^{i 0} \in \mathcal{F}$. Daraus folgt $F \cong M_{1}^{*} \cong M_{1} \subseteq F$ oder $M_{1}^{*}=F$, was gleichbedeutend mit $E I 1=1$ ist.

Wir geben noch ein Kriterium: Es sei $\left\{z_{n}\right\}$ eine Folge von Punkten auf $R$, für welche die Summe 


$$
\sum_{n=1}^{\infty} g\left(z, z_{n}\right)
$$

konvergent ist und $S\left(z_{n}, \rho\right)$ sei der hyperbolische Kreis mit dem Zentrum in $z_{n}$ und mit dem hyperbolischen Radius $\rho$. Jede abgeschlossene Menge $K$ welche in $\bigcup_{n=1}^{\infty} S\left(z_{n}, \rho\right)$ enthalten ist, ist eine dünne Menge. Wir nehmen als Menge $K^{*}$ die Menge $\bigcup_{n=1}^{\infty} S\left(z_{n}, \rho\right)$ und sei $\xi(z)$ gleich 1 auf $R-K^{*}$ und gleich 0 auf $K^{*}$. Es seien $\left\{t_{\nu}\right\}$ die Punkte, für welche $\varphi\left(t_{\nu}\right)=z_{n}(n=1,2, \ldots)$ ist. Man sieht leicht, dass

$$
\sum_{\nu=1}^{\infty} \log \frac{1}{\left|t_{\nu}\right|}=\sum_{n=1}^{\infty} g\left(\varphi(0), z_{n}\right)<\infty
$$

ist, woraus die Konvergenz des Blaschke-Produktes

$$
\pi(t)=\prod_{\nu=1}^{\infty} \frac{t-t_{\nu}}{t \bar{t}_{\nu}-1} \frac{\bar{t}_{\nu}}{\left|t_{\nu}\right|}
$$

folgt. Es sei $e^{i 0} \in \widetilde{F}$ ein Punkt, wo $|\pi(t)|$ den Winkelgrenzwert 1 hat. Aus dem Prinzip des hyperbolischen Masses folgt $\mid \pi(t) ! \leqq$ th $\rho<1$ für jedes $t \in K^{*}$, denn es ist $\pi\left(t_{*}\right)=0$. Daraus folgt

$$
\xi\left(\varphi\left(e^{i \theta}\right)\right)=1
$$

und $e^{i 0} \in \mathfrak{M}_{1}^{*}$. Da der Betrag des Blaschke-Produktes fast überall den Winkelgrenzwert 1 hat, so ergibt sich, das $\mathfrak{M}_{1}^{*}$ das Mass $2 \pi$ hat und aus $\mathfrak{M}_{1}^{*} \cong \mathfrak{R}_{1} \subseteq \pi$ folgt $M_{1}=F$ mit Ausnahme einer Menge vom Masse Null. Das ist aber gleich. bedeutend mit $E I 1=1$ und die Menge $K$ ist dünn.

SATz 12. Es sei $R \in U$ und $K$ eine dünne Menge auf $R$. Wenigstens eine Komponente der offenen Menge $R-K$ gehört der Klasse $U$ an.

Es sei $K^{*} \supseteqq K$, so dass $\Delta=R-K^{*}$ analytischen Rand hat und $E I 1=1$ ist. Es sei

$$
\Delta=\bigcup_{i}^{\prime}
$$

wo $R_{\nu}^{\prime}$ die Komponente der Menge $\Delta$ sind. Betrachten wir die in 11. eingeführten Mengen $M_{(v) 1}$, so folgt aus

$$
\begin{aligned}
& M_{1}=\bigcup_{\nu} M_{(\nu) 1}, \\
& F=\bigcup_{\nu} M_{(\nu) 1},
\end{aligned}
$$

wo die Gleichheiten mit Aushahme einer Menge vom Masse Null anzunehmen 
sind. Es sei $M \leqq F$ eine unteilbare Menge. Dann gibt es ein $\nu$, so dass $M \subseteq M_{(\nu) 1}$ mit Ausnahme einer Menge vom Masse Null. Wenn wir $R_{\nu}^{\prime}=R^{\prime}$ stellen, so ist $I^{*} M$ nach 12 . eine unteilbare Menge auf dem Rande der Fläche $R^{\prime}$ und $R^{\prime} \in U$. Die Komponente von $R-K$ welche $R^{\prime}$ enthält ist eine Fortsetzung von $R^{\prime}$ und nach dem Satze 11 folgt, dass sie auch der Klasse $U$ angehört, was $\mathrm{zu}$ beweisen war.

SAtz 12'. Es sei $R \in U_{H D}$ und $K$ eine abgeschlossene Menge auf $R$, für die

$$
\inf _{z \in K} g\left(z, z_{0}\right)=\varepsilon_{K}>0
$$

ist. Wenigstens eine Komponente der offenen Menge $R-K$ gehört der Klasse $U_{I I I)}$ an.

Es sei $\Delta$ die offene Menge $g\left(z, z_{0}\right)<\varepsilon$ und $M$ die $H D$-unteilbare Menge auf dem Rande von $R$. Es ist zu ersehen, genau wie oben, dass $\Delta$ eine Komponente $R^{\prime}$, enthält, so dass $M \cap M_{(i) 1}$ positivès Mass hat. Weiter folgt, dass $I^{*} M$ positives Mass hat, wo $I$ in Bezug auf das Paar $\left(R_{y}^{\prime}, R\right)$ zu nehmen ist. Nun ist $M^{\prime}=I^{*} M$ eine freie Menge in Bezug auf $\partial R_{!}^{\prime}$, denn $u(z)=\varepsilon-g\left(z, z_{0}\right)$ ist eine harmonische Funktion mit endlichem Dirichlet-Integral auf $R_{\nu}^{\prime}$, Null auf $\partial R_{\nu}^{\prime}$ und $u\left(\varphi^{\prime}\left(t^{\prime}\right)\right)$ hat fast überall auf $\mathfrak{l}_{\mathfrak{l}^{\prime}}$ den Winkelgrenzwert $\varepsilon$. Nach dem Hilfssatz 8 folgt, dass $M^{\prime}$ eine $H D$-unteilbare Menge ist und $R_{\nu}^{\prime} \in U_{H D}$. Nun ist $R_{\nu}^{\prime}$ in einer Komponente der Menge $R-K$ enthalten und nach dem Satze 11 folgt, dass diese Komponente auch der Klasse $U_{H D}$ angehört, was zu beweisen war.

Aus den Sätzen $7,12,12^{\prime}$ folgt unmittelbar der Satz von Kuramochi ([6], Theorem 1, [4], [2]) welcher lautet: Es sei $R^{\prime}=R-K$, wo $K$ eine kompakte Menge von $R$ ist. Ist $R \in O_{H B}-O_{G}$ (bzw. $R \in O_{H D}-O_{G}$ ), so ist $R^{\prime} \in O_{A B}$ (bzw. $\left.R^{\prime} \in O_{A D}\right)$.

22. Definition 5. Die Riemannsche Fläche $R$ gehört der Klasse $O_{H B_{n}}$ (bzw. $\left.O_{I I n_{n}}\right)(1 \leqq n \leqq \infty)$ an, wenn der ganze ideale Rand von $R$ aus höchstens $n H B$ unteilbaren (bzw. maximalen HD-unteilbaren) Mengen besteht.

Eine solche Riemannsche Fläche enthält auf dem idealen Rande keine Menge $M$, die mit der Gesamtheit ihrer Teilmengen nicht $H B$ (bzw. $H \dot{D}$ )unteilbare Mengen sind.

SATZ 13. Es ist 


$$
\begin{aligned}
& O_{H B}-O_{G}=O_{H B_{1}} ; O_{H D}-O_{G}=O_{H D_{1}} \\
& O_{H B_{n}} \cong O_{H B_{n+1}} \subseteq O_{H B_{\infty}} \subseteq U_{H B} ; O_{H D_{n}} \subseteq O_{H D_{n+1}} \subseteq O_{H D_{\infty}} \subseteq U_{I I D} \\
& O_{H B_{n}} \subseteq O_{H D_{n}} \quad(1 \leqq n \leqq \infty) .
\end{aligned}
$$

Alle diese Beziehungen sind evident, mit Ausnahme von

$$
O_{H D}-O_{G}=O_{H n_{1}}
$$

welche aus dem Hilfssatze 5 oder aus dem Satze 9 folgt.

Enthält der ideale Rand von $R$ eine Menge $M$, für welche sämtliche Teilmengen nicht HY-unteilbar sind $(Y=B, D)$, so werden wir zeigen, dass die Dimension des linearen Raumes $H Y$ unendlich ist.

Erstens kann man eine Funktion $u_{1}(z) \in H Y$ finden, so dass $u_{i}(c(t))$ nicht fast überall auf $\mathfrak{M}$ einen konstanten Winkelgrenzwert besitzt. Dann gibt es zwei reelle Zahlen $c^{\prime}, c^{\prime \prime}, c^{\prime}<c^{\prime \prime}$, so dass

$$
\begin{array}{lll}
u_{1}\left(\varphi\left(e^{i \theta}\right)\right)<c^{\prime} & \text { für } & e^{i \theta} \in \mathfrak{M}_{1}^{\prime} \subset \mathfrak{X}, \\
u_{1}\left(\varphi\left(e^{i \theta}\right)\right)>c^{\prime \prime} & \text { für } & e^{i 0} \in \mathfrak{M}_{1} \subset \mathfrak{M}
\end{array}
$$

ist, wo beide Mengen $\mathfrak{M}_{1}^{\prime}, \mathfrak{M}_{1}$ positives Mass haben. Es sei $\bar{u}_{1}(z)$ die obere Hülle von $u_{1}(z)$ und $c^{\prime}$ und $\underline{u}_{1}(z)$ die untere Hülle von $\bar{u}_{1}(z)$ und $c^{\prime \prime}$. Wir stellen

$$
v_{1}(z)=\frac{\underline{u}_{1}(z)-c^{\prime}}{c^{\prime \prime}-c^{\prime}} \in H Y
$$

und es ist $v_{1}\left(\varphi\left(e^{i \theta}\right)\right)=1$ für fast alle $e^{i 0} \in \mathfrak{M}_{1}$ und $v_{1}\left(\varphi\left(e^{i 0}\right)\right)=0$ für fast alle $e^{i \theta} \in \mathfrak{M}_{1}^{\prime}$. Da nach der Vorausstezung $M_{1}$ nicht $H Y$-unteilbar ist, so kann man eine Funktion $u_{2}(z) \in H Y$ finden $0<u_{2}(z)<1$, so dass $u_{2}(\varphi(t))$ nicht fast überall auf $\mathfrak{M}_{1}$ einen konstanten Winkelgrenzwert hat. Es sei $u_{2}^{\prime}(z)$ die untere Hülle von $u_{2}(z)$ und $v_{1}(z)$. Es ist $u_{2}^{\prime}(z) \in H Y$ und $u_{2}^{\prime}\left(\varphi\left(e^{i \theta}\right)\right)=0$ fast überall auf $\mathfrak{M}_{1}^{\prime}$ und $u_{2}^{\prime}\left(\varphi\left(e^{i 0}\right)\right)=u_{2}\left(\varphi\left(e^{i 0}\right)\right)$ fast überall auf $\mathfrak{M}_{1}$. Genau wie oben bilden wir jetzt eine Funktion $v_{2}(z) \in H Y$, für die $v_{2}\left(\varphi\left(e^{i \theta}\right)\right)=0$ fast überall auf $\mathfrak{R}_{1}^{\prime} \cup \mathfrak{M}_{2}^{\prime}$ und $v_{2}\left(\varphi\left(e^{i 0}\right)\right)=1$ fast überall auf $\mathfrak{M}_{2}$ gilt, wo $\mathfrak{M}_{1} \supseteqq \mathfrak{M}_{2} \cup \mathfrak{M}_{2}^{\prime}$ ist und $\mathfrak{M}_{2}, \mathfrak{M}_{2}^{\prime}$ positives Mass haben. Die Funktionen $v_{1}(z), v_{2}(z)$ sind offenbar linear unabhängig. Man führt dieses Verfahren fort und erhält somit eine Folge $\left\{v_{n}(z)\right\}$ von linear unabhängigen Funktionen aus der Klasse $H Y$, womit man bewiesen hat, dass die Dimension des linearen Raumes $H Y$ unendlich ist.

Enthält der ideale Rand von R keine Menge $M$, für welche sämtliche Teilmengen nicht $H Y$ unteilbar sind, so ist die Dimension des Raumes $H Y$ gleich der 
Zahl der maximalen HY-unteilbaren Mengen.

Es sei ersterıs die Zahl der maximalen $H Y$-unteilbaren Mengen gleich $n<\infty$ und seien $M_{\nu}(\nu=1,2, \ldots, n)$ diese Mengen. Ist $u(z) \in H Y$, so hat $u(\varphi(t))$

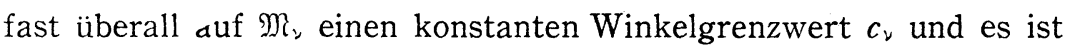

$$
u(z)=\sum_{\nu=1}^{n} c_{\nu} \omega\left(z, M_{\nu}, R\right)
$$

Wir werde zeigen, dass $\omega\left(z, M_{\nu}, R\right) \in H Y$. Für $Y=B$ ist das einleuchtend. Für $Y=D$ ist nach Satz $10 \omega\left(z, M_{\nu}, R\right) \in H D$. Man kann somit eine Folge von Funktionen $\left\{u_{k}(z)\right\}$ finden, so dass

$$
\begin{aligned}
& \omega\left(z, M_{\nu}, R\right)=\lim _{k \rightarrow \infty} u_{k}(z), \\
& u_{k}(z) \geqslant u_{k+1}(z) \geqslant 0, \quad \boldsymbol{u}_{k}(z) \in H D
\end{aligned}
$$

ist. Es ist

$$
u_{k}(z)=\sum_{\mu=1}^{n} c_{k \mu} \omega\left(z, M_{i}, R\right)
$$

wo $c_{k} \geqslant 1$ und $\lim _{k \rightarrow \infty} c_{k \mu}=0$ für $\mu \neq \nu$ ist. Für $k>N$ ist $c_{k, \mu}<\frac{1}{2}$ für $\mu \neq \nu$, und wenn wir die obere Hülle $\bar{u}_{k}(z)$ von $u_{k}(z)$ und $\frac{1}{2}$ bilden, so ist

$$
\vec{u}_{k}(z)=\frac{1}{2}+\left(c_{k \nu}-\begin{array}{l}
1 \\
2
\end{array}\right) \omega\left(z, M_{\nu}, R\right) \in H D .
$$

Daraus folgt

$$
\omega\left(z, M_{\nu}, R\right)=\frac{\bar{u}_{k}(z)-\frac{1}{2}}{c_{k \nu}-\frac{1}{2}} \in H D
$$

Die Funktionen $\omega\left(z, M_{\nu}, R\right)$ bilden somit eine Basis für den Raum $H Y$ und es ist $\operatorname{dim} H Y=n$.

Es sei jetzt die Zahl der maximalen $H Y$-unteilbaren Mengen unendlich und seien $M_{\nu}(\nu=1,2, \ldots, n) n$ solche Mengen. Im Falle $Y=B$ ist $\omega\left(z, M_{\nu}, R\right)$ $\in H B$ und da diese offenbar linear unabhängig sind, so ist $\operatorname{dim} H B \geqslant n$. Im Falle $Y=D$, ist $\omega\left(z, M_{v}, R\right) \in H D$ und wie oben haben wir

$$
\begin{aligned}
& \omega(z, M, R)=\lim _{k \rightarrow \infty} u_{k}(z), \\
& u_{k}(z) \geqslant u_{k+1}(z) \geqq 0, \quad \boldsymbol{u}_{k}(z) \in H D .
\end{aligned}
$$


Es ist

$$
u_{k}(z)=\sum_{\mu=1}^{n} c_{k \mu} \omega\left(z, M_{\mu}, R\right)+v_{k}(z)
$$

wo $v_{k}\left(\varphi\left(e^{i 0}\right)\right)=0$ für fast alle $e^{i 0} \in \bigcup_{\mu=1}^{n} \mathfrak{M}_{\mu}$ und

$$
c_{k \nu} \geqq 1, \quad \lim _{k \rightarrow \infty} c_{k \mu}=0 \quad(\mu \neq \nu, 1 \leqq \mu \leqq n)
$$

ist. Wenn man weiter den oberen Beweis verfolgt, so erhalten wir eine Funktion

$$
u_{\nu}^{\prime}(z)=\omega\left(z, M_{\nu}, R\right)+v_{\nu}^{\prime}(z) \in H D
$$

so dass $v_{\nu}^{\prime}\left(\varphi\left(e^{i \theta}\right)\right)=0$ für fast alle $e^{i \theta} \in \bigcup_{\mu=1}^{n} \mathfrak{M}_{\mu}$ ist. Daher folgt, dass $u_{\nu}^{\prime}(z)$ linear unabhängig sind und wir haben $\operatorname{dim} H D \geq n$. Wenn man $Y$ für $B$ oder $D$ schreibt und beachtet dass $n$ beliebig war, so folgt $\operatorname{dim} H Y=\infty$, was zu beweisen war.

Es seien $R_{\nu}^{\prime}(\nu=1,2, \ldots, n+1)$ punktfremcie Gebiete auf $R$ mit analytischem Rande welche der Klasse $S_{I I Y}(Y=B, D)$ nicht angehören. Dann gehört $R$ nicht der Klasse $O_{H Y_{n}}$.

Es seien $u_{\nu}(z) \in H Y$ auf $R_{\nu}^{\prime}, u_{\nu}(z) \geqslant 0$ in $R_{\nu}^{\prime}$ und $u_{\nu}(z)=0$ auf $\partial R_{\nu}^{\prime}$. Wir bezeichnen

$$
\Delta=\bigcup_{\nu=1}^{n+1} R^{\prime}
$$

und sei $u_{\nu}^{\prime}(z)$ gleich $u_{\nu}(z)$ in $R_{\downarrow}^{\prime}$ und gleich 0 in $\Delta-R_{\nu}^{\prime}$. Dann gehören $E u_{,}^{\prime}$, der Klasse $H Y$ auf $R$ an, wo die Operatoren $E, I$ in Bezug auf das Paar $(\Delta, R)$ sind. Ist

$$
\sum_{\nu=1}^{n+1} c_{\nu} E u_{\nu}^{\prime}=0
$$

oder

$$
\sum_{\nu=1}^{k} c_{\nu} E u_{\nu}^{\prime}=\sum_{\nu=k+1}^{n+1}\left(-c_{\nu}\right) E u_{\nu}^{\prime}
$$

wo $c_{\nu} \geqslant 0(1 \leqq \nu \leqq \bar{R}), c_{\nu} \leqq 0(k+1 \leqq \nu \leqq n+1)$ ist, so folgert man

$$
\begin{aligned}
& \sum_{\nu=1}^{k} c_{\nu} I E u_{\nu}^{\prime}=\sum_{\nu=k+1}^{n+1}\left(-c_{\nu}\right) I E u_{\nu}^{\prime}, \\
& \sum_{\nu=1}^{n+1} c_{\nu} u_{\nu}^{\prime}=0
\end{aligned}
$$


und $c_{2}=0(1 \leqq \nu \leqq n+1)$.

Die Funktionen Eu', sind also linear unabhängig und $\operatorname{dim} H Y \geq n+1$,

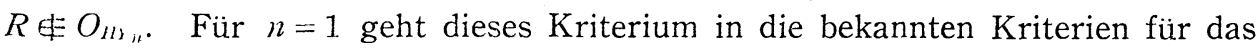
Nichtenthaltensein einer Riemannschen Fläche in der Klasse $O_{H B}$ bzw. $O_{I I D}$ über.

SATz 14. Wenn zwei Riemannsche Flächen $R^{\prime}$ bzw. $R^{\prime \prime}$ zwei kompakte Mengen $K^{\prime}$ bzu. $K^{\prime \prime}$ enthalten, so dass $R^{\prime}-K^{\prime}$ mit $R^{\prime \prime}-K^{\prime \prime}$ konform äquivalent ist, so gehören sio gleichzeitig der Klasse $O_{I I{ }_{n}}$ an $(Y=B, D)(1 \leqq n \leqq \infty)$.

Es sei $K_{0}^{\prime}$ bzw. $K_{0}^{\prime \prime}$ eine kompakte Menge auf $R^{\prime}$ bzw. $R^{\prime \prime}$, so dass $K_{0}^{\prime} \supseteqq K^{\prime}$ bzw. $K_{0}^{\prime \prime} \supseteqq K^{\prime \prime}$ und, dass $\Delta^{\prime}=R^{\prime}-K_{0}^{\prime}$ bzw. $\Delta^{\prime \prime}=R^{\prime \prime}-K_{0}^{\prime \prime}$ analytischen Rand hat. Wir können $K_{0}^{\prime}$ und $K_{0}^{\prime \prime}$ so nehmen, dass $\Delta^{\prime}$ und $\Delta^{\prime \prime}$ konform äquivalent sind. Wenn wir mit $I, E$ die Operatoren in Bezug auf das Paar $\left(\Delta^{\prime}, R^{\prime}\right)$ bezeichnen, so sind, wie bewiesen wurde, $M$ und $I^{*} M$ (bzw. $M^{\prime}$ und $E^{*} M^{\prime}$ ) gleichzeitig $H Y$-unteilbare Mengen. Daraus folgt, dass $R^{\prime} \in O_{H Y_{u}}$ genau dann, wenn $M_{1}^{\prime}$ aus $n$ maximalen $H Y$-unteilbaren Mengen besteht, woraus man unmittelbar den Satz folgert.

Dieser Satz enthält für $n=1$ den Satz von Mori [12], Royden [18] und Sario [20], dass das Enthaltensein einer Riemannschen Fläche in der Klasse $O_{i Y Y}$ eine "Randeigenschaft" ist.

SATZ 15. Es ist

$$
O_{H B_{\infty}} \subseteq O_{A B}^{0} ; O_{H D_{\infty}} \subseteq O_{A D}^{0}
$$

Es sei $R^{\prime}$ ein Gebiet mit analytischem Rande auf $R \in O_{H Y_{\infty}}$. Ist $I 1 \equiv 0$, wo $I$ in Bezug auf das Paar $\left(R^{\prime}, R\right)$ bestimmt ist, so ist nach $15 . R^{\prime} \in S O_{H B} \subseteq S O_{H \prime}$ und offenbar $R^{\prime} \in \mathrm{SO}_{A Y}$. Ist $I 1 \neq 0$ so hat die Menge $M_{1}$ positives Mass. Dann gibt es mindestens eine $H Y$-unteilbare Menge $M \cong M_{1}$ und $I^{*} M$ hat ein positives Mass. Für $Y=B$ folgt unmittelbar, dass $I^{*} M, H B$-unteilbar ist, und das Gebiet $R^{\prime}$ enthält überhaupt keine beschränkte analytische Funktion: $R^{\prime} \in S O_{A B}$. Für $Y=D$ sei $w(z)=u(z)+i v(z)$ eine eindeutige analytische Funktion mit endlichem Dirichlet-Integral auf $R^{\prime}$ und $u(z)=0$ auf $\partial R^{\prime}$. Ist $I^{*} M$ frei in Bezug auf $\partial R^{\prime}$, so folgt aus dem Hilfssatz 8, dass $I^{*} M$ eine $H D$-unteilbare $M$ enge auf dem idealen Rande von $R^{\prime}$ ist. Daraus folgt $R^{\prime} € U_{H D} \subseteq O_{A D}-O_{G}$ und $w(z)$ ist konstant. Sind alle Mengen $I^{*} M$ gebunden am relativen Rande $\partial R^{\prime}$ von $R^{\prime}$ so hat $u\left(\varphi^{\prime}\left(t^{\prime}\right)\right)$ fast überall auf $\left|t^{\prime}\right|=1$ den 'Winkelgrenzwèrt Null, woraus $u(z) \equiv 0$ und $w(z)$ $\equiv$ konstant folgt. Es ist also $R^{\prime} \in S O_{A D}$, was $z$ u beweisen war. 
Dieser Satz enthält den Satz von Kuroda [8], laut welches $O_{I I B} \cong O_{{ }^{\prime} B}$ ist. Für $O_{i / B_{\infty}}$ haben wir sogar mehr bewiesen. Es sei $w(z)$ eine eindeutige meromorphe Funktion auf $R \in O_{\| B_{\infty}}$ und $R^{\prime}$ eine Komponente der offenen Menge $w(z)-w_{0} \mid<\rho$, wo $w_{0}$ und $\rho$ beliebig sind (für $w_{0}=0$ nehmen wir $|w(z)|>\rho$ ). Dann ist zweifellos $R^{\prime} \in S O_{I i R}$, denn im entgegengesetzten Falle ist $R^{\prime} \in O_{A B}$ im Widerspruch mit der Existenz von $w(z)$. Daraus folgt, wie Tsuji gezeigt hat ([23], Theorem 2, [11]), dass $w(z), z \in R^{\prime}$, alle Punkte des Kreises $\left|w-w_{0}\right|<\rho$ mit Ausnahme einer Menge von der Kapazität Null überdeckt. Daraus folgert man sofort, dass wenn eine Riemannsche Fläche $R \in O_{\|_{i i_{3}}}$ eine beliebige Riemannsche Fläche $R_{1}$ überlagert, so ist die Menge der nicht überdeckten Punkte von der Kapazität Null.

23. Es sei $\widetilde{R}$ eine Riemannsche Fläche welche die Riemannsche Fläche $R$ überlagert und sei $z=f(\widetilde{z})$ die Spurabbildung. Mann sagt, dass die Überlagerung vollständig ist ([22], Seite 125, recouvrement Riemannien total), wenn für jede Folge $\left\{\widetilde{z}_{n}\right\}, \widetilde{z}_{n} \in \widetilde{R}$, welche gegen den idealen Rand der Fläche $\widetilde{R}$ konvergiert, die Folge $\left\{z_{n}=f\left(\widetilde{z}_{n}\right)\right\}$ der Überlagerten Punkte gegen den idealen Rand der Fläche $R$ konvergiert. Es ist bewiesen worden ([22], Seite 126), dass es eine ganze Zahl $g$ gibt, so dass jeder Punkt $z \in R$ von genau $g$ Punkten aus $\tilde{R}$ überlagert wird. Man nennt die Zahl $g$ den Grad der Überlagerung. Wenn die überlagerung von $R$ durch $\widetilde{R}$ vollständig ist, so gibt es zwischen den Eigenschaften der Flächen $R$ und $\widetilde{R}$ einen engen Zusammenhang.

HilfsSATZ 9. Es sei $z=f(\widetilde{z})$ eine vollständige Überlagerung vom Grade $g$ der Riemannschen Fläche $R$ durch eine Riemannsche Fläche $\widetilde{R} . \quad$ Ist $u(z)$ eine minimale Funktion auf $R$ in der Klasse $H B$ (bzu. $\underline{H D}$ ) so gibt es $k(k \leqq g)$ Funktionen auf $\widetilde{R}, \widetilde{u}_{,}(\widetilde{z}),\{\nu=1,2, \ldots, k \leqq g\}$ u'elche minimal in der Klasse $H B$ (bzw. HD) auf $\widetilde{R}$ sind, und für welche

$$
\widetilde{u}_{2}(\tilde{z}) \leqq \tilde{u}(\tilde{z})=u(f(\tilde{z}))
$$

ist. Für diese Funktionen ist

$$
\sum_{v=1}^{k} \tilde{u}_{u}(\widetilde{z})=\tilde{u}(\tilde{z})
$$

Da $u(z)$ eine in der Klasse $H B$ (bzw. $H D$ ) minimale Funktion ist, so gibt es eine $H B$ (bzw. maximale $H D$ ) unteilbare Menge $M$, so dass $u(z)=c \omega(z, M, R)$ ist. Mann sieht leicht, dass wenn $u(z) \in H B$ (bzw. $H D$ ) ist, so ist $\tilde{u}(\bar{z}) \in H B$ 
(bzw. $H D$ ). Es sei $\tilde{\zeta}(\tilde{t})$ die eineindeutige und konforme Abbildung des Kreises $|\tilde{t}|<1$ auf die universelle Überlagerungsfläche von $\widetilde{R}$ und $\widetilde{\mathfrak{X}}$ die Menge der Punkte $e^{i \tilde{\hat{n}}}$, wo $\widetilde{u}(\widetilde{\zeta}(\hat{t}))$ den Winkelgrenzwert $c$ hat. Es sei $c^{\prime}<c$ und $\overline{\bar{u}}(\tilde{z})$ die obere Hülle von $\tilde{u}(\tilde{z})$ und $c^{\prime} ; \overline{\bar{u}}(\tilde{z}) \in H B$ (bzw. $\underline{H D}$ ). Es ist ersichtilich, dass $\tilde{\tilde{u}}(\widetilde{z})$ denselben Wert annimmt in zwei Punkten welche denselbem Spurpunkt haben, denn es genügt, bei der Konstruktion von $\tilde{u}(\widetilde{z})$, eine Ausschöpfung der Fläche $R$ zu wählen, und sie in eine Ausschöpfung der Fläche $\widetilde{R}$ durchzudrücken. Deshalb ist die Funktion $\bar{u}(z)=\overline{\tilde{u}}\left(f^{-1}(z)\right)$ eine eindeutige Funktion. Es ist offenbar

$$
c \frac{\overline{\bar{u}}(\tilde{z})-c^{\prime}}{c-c^{\prime}} \leqq \tilde{u}(\tilde{z})
$$

und daraus folgt

$$
c \frac{\bar{u}(z)-c^{\prime}}{c-c^{\prime}} \leqq u(z)
$$

und, da

$$
\left.c \frac{\bar{u}(z)-c^{\prime}}{c-c^{\prime}} \in H B \quad \text { (bzw. } \underline{H D}\right),
$$

so ist

$$
c \frac{\bar{u}(z)-c^{\prime}}{c-c^{\prime}}=c^{\prime \prime} u(z)
$$

oder

$$
c \frac{\overline{\tilde{u}}(\tilde{\varphi}(\tilde{t}))-c^{\prime}}{c-c^{\prime}}=c^{\prime \prime} \tilde{u}(\widetilde{\varphi}(\tilde{t})) .
$$

Daraus sieht man, dass $\widetilde{u}(\widetilde{\varphi}(\tilde{t}))$ fast überall den Winkelgrenzwert 0 hat wo der Winkelgrenzwert kleiner als $c^{\prime}$ ist. Da $c^{\prime}<c$ beliebig war, so ergibt sich $\tilde{u}(\widetilde{z})$ $=c \omega(\widetilde{z}, \widetilde{M}, \widetilde{R})$. Die Menge $\tilde{M}$ enthält keine Teilmenge $\tilde{M}^{\prime}$ welche gemeinsam mit allen ihren Teilmengen nicht $H B$ (bzw. $H D$ )-unteilbar ist. Denn würde $\tilde{M}^{\prime} \subseteq \tilde{M}$ diese Eigenschaft besitzen, so könnte man, wie leicht zu beweisen ist, eine Teilmenge $\widetilde{M}^{\prime \prime}$ von $\tilde{M}^{\prime}$ finden, für die $\omega\left(\widetilde{z}, \widetilde{M}^{\prime \prime}, \widetilde{R}\right) \in H B$ (bzw. $\underline{H D}$ ) und

$$
\omega\left(\widetilde{z}_{0 \nu}, \widetilde{M}^{\prime \prime}, \widetilde{R}\right)<\frac{1}{g} \omega\left(\widetilde{z}_{0 \nu}, \tilde{M}, \widetilde{R}\right)
$$

in allen Punkten $\tilde{z}_{0}$, ist die sich in einem bestimmten Punkt $z_{0}$ projizieren. Es sei

$$
\widetilde{v}(\widetilde{z})=\sum_{\nu=1}^{g} \widetilde{\omega}\left(\widetilde{z}_{\nu}, \tilde{M}_{*}^{\prime \prime}, \widetilde{R}\right)
$$

wo die Summe sich über alle $\widetilde{z}_{\downarrow}$ erstreckt für welche $f\left(\widetilde{z}_{\downarrow}\right)=f(\widetilde{z})$ ist. Da $\widetilde{v}(\widetilde{z})$ 
offenbar in zwei Punkte mit demselben Spurpunkt, denselben Wert hat, so ist $v(z)=\widetilde{v}\left(f^{-1}(z)\right)$ eine eindeutige harmonische Funktion. Da $\widetilde{v}(\widetilde{z})$ der Klasse $H B$ (bzw. $H D$ ) angehört, so gehört auch $v(z)$ dieser Klasse an. Es ist

$$
v\left(z_{0}\right)=\widetilde{v}\left(f^{-1}\left(z_{0}\right)\right)=\sum_{\nu=1}^{y} \omega\left(\widetilde{z}_{0 \nu}, \widetilde{M}^{\prime \prime}, \widetilde{R}\right)<\frac{1}{g} \sum_{\nu=1}^{g} \omega\left(\widetilde{z}_{0_{\nu}}, \tilde{M}, \widetilde{R}\right)=\omega\left(z_{0}, M, R\right)
$$

Aus $\omega\left(\widetilde{z}, \widetilde{M}^{\prime \prime}, \widetilde{R}\right) \leqq \omega(\widetilde{z}, \tilde{M}, \widetilde{R})$ folgt $v(z) \leqq g \omega(z, M, R)$ und da $\omega(z, M, R)$ eine minimale Funktion in der Klasse $H B$ (bzw. $H D$ ) ist, so folgt $v(z)$ $=c^{\prime \prime \prime} g \omega(z, M, R)$. Im Punkte $z_{0}$ ist

$$
c^{\prime \prime \prime} g \omega\left(z_{0}, M, R\right)=v\left(z_{3}\right)<\omega\left(z_{0}, M, R\right),
$$

was $c^{\prime \prime \prime} g<.1$ als Folge hat. Es ist aber

$$
1=\sup _{\widetilde{z} \in \widetilde{R}} \omega\left(\widetilde{z}, \widetilde{M}^{\prime \prime}, \widetilde{R}\right) \leqq \sup _{\widetilde{z} \in \widetilde{R}} \widetilde{v}(\widetilde{z})=\sup _{z \in i} v(z)=c^{\prime \prime \prime} g<1
$$

Der gefundene Widerspruch zeigt, dass $\tilde{M}$ keine Teilmenge $\tilde{M}^{\prime}$ enthält welche mit ihren sämtlichen Teilmengen nicht $H B$ (bzw. $H D$ ) unteilbar ist. Es ist also

$$
\widetilde{M}=\bigcup_{\mu} \tilde{M}_{\mu}
$$

wo $\tilde{M}_{\mu}$ punktfremd und $H B$ (bzw. maximale $H D$ )-unteilbare Mengen sind. Wie oben sieht man, dass

$$
\sum_{\nu=1}^{g} \omega\left(\widetilde{z}_{\nu}, \tilde{M}_{\mu}, \widetilde{R}\right)=c_{\mu} g \omega(\widetilde{z}, \tilde{M}, \widetilde{R})
$$

ist. Es ist

$$
1=\sup _{\widetilde{z} \in \widetilde{R}} \omega\left(\widetilde{z}, \widetilde{M}_{\mu}, \widetilde{R}\right) \leqq c_{\mu} g
$$

und

$$
\sum_{\mu=1}^{k} \sum_{\nu=1}^{g} \omega\left(\widetilde{z}_{\nu}, \tilde{M}_{\mu}, \widetilde{R}\right)=\sum_{\mu=1}^{k} c_{\mu} g \omega(\widetilde{z}, \tilde{M}, \widetilde{R})
$$

und daher ist

$$
\sum_{i=1}^{g} \omega\left(\widetilde{z}_{\nu}, \bigcup_{n=1}^{k} \tilde{M}_{\mu}, \widetilde{R}\right) \geq k \omega(\widetilde{z}, \tilde{M}, \widetilde{R})
$$

Nun ist aber

$$
g \omega(\widetilde{z}, \tilde{M}, \widetilde{R}) \gg \sum_{\nu=1}^{g} \omega\left(\widetilde{z}_{\nu}, \bigcup_{\mu=1}^{k} \tilde{M}_{\mu}, \widetilde{R}\right) \geq k \omega(\widetilde{z}, \tilde{M}, \widetilde{R}),
$$

woraus $k \leqq g$ folgt. Es ist also 


$$
\tilde{M}=\bigcup_{\mu=1}^{k} \tilde{M}_{\mu}
$$

und

$$
\omega(\tilde{z}, \tilde{M}, \widetilde{R})=\sum_{\mu=1}^{k} \omega\left(\tilde{z}, \tilde{M}_{\mu}, \widetilde{R}\right)
$$

was $\mathrm{zu}$ beweisen war.

Aus diesem Hilfssatz folgert man unmittelbar den

SATZ 16. Es sei $z=f(\tilde{z})$ eine vollständige Überlagerung vom Grade $g$ der Riemannschen Fläche $R$ durch eine Riemannsche Fläche $\widetilde{R}_{0}$. Gehört $R$ der Klasse $U_{I I B}$ (bzw. $\left.U_{I I D}\right)$ an, so gehört auch $\widetilde{R}$ dieser Klasse an. Gehört $R$ der Klasse $O_{H B_{n}}\left(\right.$ bzw. $\left.O_{H D_{n}}\right)(1 \leqq n \leqq \infty)$ an, so gehört $\widetilde{R} \operatorname{der}$ Klasse $O_{H B_{n q}}\left(b z w . O_{H D_{n g}}\right)$ an.

24. Wir wollen einige Beispiele von Riemannschen Flächen aus der Klasse $U_{H Y}$ angeben. Es sei $R_{0} \in O_{H Y}-O_{G}$ und $K$ eine Kreisscheibe auf $R$. Nach den Sätzen $12,12^{\prime}$ gehört $R_{0}-K$ der Klasse $U_{H Y}$ an. Es sei $R_{1}$ eine andere beliebige Riemannsche Fläche uud $K_{1}$ eine Kreisscheibe auf $R_{1}$. Wenn man die Kreisscheibe $K_{1}$ entfernt und an das so entstandene Loch die Fläche $R_{0}-K$ klebt, so dass der Rand von $K$ an den Rand von $K_{1}$ geklebt wird, so entsteht eine neue Riemannsche Fläche $R$, welche $R^{\prime}=R_{0}-K$ als Gebiet enthält. Die Menge $M_{1}^{\prime}$ von $R^{\prime}$ besteht genau aus einer $H Y$-unteilbaren Menge. Der ideale Rand von $R$ enthält die $H Y$-unteilbare Menge $M=I^{*} M^{\prime}$ und $R$ gehört somit der Klasse $U_{H Y}$ an. Indem man in $R_{1}$ mehrere Löcher macht und an jedes ein Exemplar $R_{0}-K$ anklebt, so entsteht eine Riemannsche Fläche die auf dem idealen Rande beliebig viele (sogar unendlich viele) $H Y$-unteilbare Mengen enthält. War $R_{1}$ die $z$-Ebene, so enthält man Beispiele von Flächen aus der Klasse $O_{H^{\prime}{ }_{n}}-O_{H^{\prime}{ }_{n-1}}$ oder $O_{I r_{\infty}}-\bigcup_{n=1}^{\infty} O_{H r_{n}}$. Es ist somit

$$
O_{H y_{n=1}} \subset O_{H \gamma_{n}}, \bigcup_{n=1}^{\infty} O_{H \gamma_{n}} \subset O_{H Y_{c o}} .
$$

In dem obigen Beispielen befindet sich auf einer idealen Randkomponente höchstens eine $H Y$-unteilbare Menge. Das ist aber nicht immer so. Es sei $R_{0} \in O_{H Y}-O_{G}$ so, dass $R_{0}$ eine einzig eideale Randkomponente besitzt. Wir zeichnen auf $R_{0}$ eine Folge von kompakten analytischen Jordanbogen $\left\{l_{n}\right\}$, so dass

$$
l_{n} \cap l_{m}=\phi
$$


für $n \neq m$ und dass für ein beliebiges Kompakt $K \subset R_{0}$,

$$
l_{n} \cap K=\phi
$$

für genügend grosse $n$ ist. Wir bezeichnen mit

$$
R^{\prime}=R_{0}-\bigcup_{n=1}^{\infty} l_{n}
$$

und nehmen die Folge $\left\{l_{n}\right\}$ so, dass $R^{\prime}$ nicht der Klasse $\mathrm{SO}_{I \prime}$ angehören soll. Wir nehmen $g$ solcher Exemplare von $R^{\prime}$ und numerotieren sie mit $R_{1}^{\prime}, R_{2}^{\prime}, \ldots$, $R_{g}^{\prime}$. Wir kleben jetzt diese Exemplare nach folgender Regel: jedes $l_{n}$ hat zwei Ufer, welche wir mit $l_{n}^{+}$und $l_{n}^{-}$bezeichnen. Wir kleben $l_{n}^{+}$von $R_{j}^{\prime}$ mit $l_{n}^{-}$von $R_{j+1}^{\prime}(g+1=1)$ zusammen für alle $n$. Damit entsteht eine Riemannsche Fläche $R$. Sie hat eine einzige ideale Randkomponente und enthält $R_{j}^{\prime}(1 \leqq j \leqq g)$ als punktfremde Gebiete, die der Klasse $S O_{I i}$ nicht angehören. Nach dem oben angegebenen Kriterium 22 gehört $R$ nicht der Klasse $O_{n / /-1}$ an. Nun bildet $R$ eine vollständige Überlagerung vom Grade $g$ der Fläche $R_{0}$. Nach dem Satze 16 ist dann $R \in O_{H 1 \%}$. Daraus folgt $R \in O_{I_{1} \varphi_{4}}-O_{H^{\prime} \gamma_{-1}}$ und die ideale Randkomporent besteht genau aus $g$ (maximalen) $H Y$-unteilbaren Mengen.

Wir nehmen zwei Exemplare von $R^{\prime}$ die wir mit $R^{\prime}$ und $R^{\prime \prime}$ bezeichnen und kleben den Ufer $l_{2 k+1}^{+}$von $R^{\prime}$ mit $l_{2 k+1}^{-}$von $R^{\prime \prime}$ und $l_{2 k+1}^{-}$von $R^{\prime}$ mit $l_{2 k+1}^{+}$von $R^{\prime \prime}$ für alle $k, 1 \leqq k<\infty$. Wir erhalten somit eine Riemannsche Fläche, die wir mit $\bar{R}$ bezeichnen und nehmen unendlich viele solcher Exemplare die wir mit $\bar{R}_{n}$ numerotieren $(-\infty<n<+\infty)$. Jedes Exemplar hat zwei Jordansche Bogen $l_{2 k}: l_{2 k}^{\prime}, l_{2 k}^{\prime \prime}$. Wir kleben jetzt das Exemplar $\bar{R}_{n}$ längs $l_{2 k}^{\prime}$ mit $\bar{R}_{n+1}$ längs $l_{2 k}^{\prime \prime}$ zusammen $(1 \leqq k<\infty)(-\infty<n<+\infty)$; dabei werden die Ufer genau wie oben geklebt. Somit entsteht eine Riemannsche Fläche $R$, welche eine einzige ideale Randkomponente besitzt. Wir bezeichnen mit $\Delta$ die Vereinigung aller Gebiete $R^{\prime}$ und $R^{\prime \prime}$ und nehmen die Operatoren $E, I$ in Bezug auf das Paar $(\Delta, R)$. Jedes Gebiet $R^{\prime}, R^{\prime \prime}$ hat als idealen Rand genau eine $H Y$-unteilbare Menge, die wir mit $M^{\prime}$ bzw. $M^{\prime \prime}$ bezeichnen. Dann sind $E^{*} M^{\prime}$ bzw. $E^{*} M^{\prime \prime}$ auch $H Y$-unteilbare Mengen und der ideale Rand von $R$ enthält unendlich viele $H Y$-unteilbare Mengen.

Es ist leicht zu sehen, dass $E I 1(z)$ auf alle $\bar{R}_{n}$ identisch ist. Deshalb, wenn wir $R^{\prime}$ und $R^{\prime \prime}$ in $\bar{R}_{n}$ längs der gebliebenen Bogen $\left\{l_{2 k}^{\prime}\right\}\left\{l_{2 k}^{\prime \prime}\right\}$ zusammenkleben. so wird $E I 1(z)$ eine harmonische Funktion auf der so entstandenen Fläche sein. 
Diese Fläche ist aber nichts anderes, als das obengegebene Beispiel von einer Fläche aus der Klasse $O_{H Y_{2}}-O_{H Y_{1}}$. Daraus kann man folgern, dass $E I 1(z) \equiv 1$ ist. Das heisst, dass die Mengen $E^{*} M^{\prime}, E^{*} M^{\prime \prime}$, für alle $M^{\prime}, M^{\prime \prime}$ den ganzen idealen Rande der Fläche $R$ ausmachen. Die Fläche $R$ gehört also der Klasse

$$
O_{H Y_{\infty}}-\bigcup_{n=1}^{\infty} O_{n Y_{n}}
$$

an.

\section{LITERATUR}

[1] Bader, R.-Parreau, M., Domaines non compacts et classification des surfaces de Riemann, Comptes Rendus, Paris, 232 (1951), 138-139.

[2] Cornea, A., On the behaviour of analytic functions in the neighbourhood of the boundary of a Riemann surface, Nagoya Math. J., 12 (1957), 55-58.

[ 3 ] Heins, M., On the Lindelöf principle, Ann. of Math., 61 (1955), 440-473.

[ 4 ] Kuramochi, Z., On covering surfaces, Osaka Math. J., 5 (1953), 155-201.

[5] Kuramochi, Z., Relations between harmonic dimensions, Proc. Japan Acad., 30 (1954), $576-580$.

[6] Kuramochi, Z., On the behaviour of analytic functions on abstract Riemann surfaces, Osaka Math. J., 7 (1955), 109-127.

[7] Kuroda, T., A property of some open Riemann surfaces and its application, Nagoya Math. J., 6 (1953), 77-84.

[8] Kuroda, T., On analytic functions on some Riemann surfaces, Nagoya Math. J., 10 (1956), 27-50.

[9] Lusin, N.-Priwaloff, I., Sur l'unicité et la multiplicité des fonctions analytiques, Ann. Sci. Ecole. Norm. Sup., 3 Série, 42 (1925), 143-191.

[10] Martin, R. S., Minimal positive harmonic functions, Trans. Amer. Math. Soc., 49 (1941), 137-172.

[11] Mori, A., On Riemann surfaces, on which no bounded harmonic function exists, J. Math. Soc. Japan, 3 (1951), 285-289.

[12] Mori, A., On the existence of harmonic functions on a Riemann surface, J. Fac. Sci. Tokyo, 6 (1951), 247-257.

[13] Mori, A., An imbedding theorem on finite covering surfaces of the Riemann sphere, J. Math. Soc. Japan, 5 (1ᄃ53), 263-268.

[14] Myrberg, P. J., Über die analytische Fortsetzung von beschränkten Funktionen, Ann. Acad. Sci. Fenn., 58 (1949).

[15] Nevanlinna, R., Eindeutige analytische Funktionen, Zweite Auflage, Springer Verlag (1953).

[16] Nevanlinna, R., Uniformisierung, Springer Verlag (1953).

[17] Parreau, M., Sur les moyennes des fonctions harmoniques et analytiques et la classification des surfaces de Riemann, Ann. Inst. Fourier, 3 (1952), 103-198.

[18] Royden, H. L., Some remarks on open Riemann surfaces, Ann. Acad. Sci. Fenn., 85 (1951). 
[19] Sario, L., Sur la classification des surfaces de Riemann, Den 11 skandinaviske matematikerkongress i Trondheim 22-25, August 1949.

[20] Sario, L., A linear operator method on arbitrary Riemann surfaces, Trans. Amer. Math. Soc., 72 (1952), 281-295.

[21] Stoïlow, S., Sur les fonctions analytiques dont les surfaces de Riemann ont des frontières totalement discontinues, Mathematica, 12 (1936), 123-138.

[22] Stoïlow, S., Leçons sur les principes topologiques de la théorie des fonctions analytiques, Deuxième édition, Gauthier Villars (1956).

[23] Tsuji, M., Function of U.class and its applications, Jour. Math. Soc. Japan, 7 (1955), 166-176.

[24] Virtanen, K. I., Über die Existenz von beschränkten harmonischen Funktionen auf offenen Riemannschen Flächen, Ann. Acad. Sci. Fenn., 75 (1950).

Mathematisches Institut,

Rumänische Akademie 